\title{
The Long-Term Effects of Management and Technology Transfers
}

\author{
By Michela Giorcelli
}

\section{Online Appendix - Not For Publication}

\section{A. Additional Figures and Tables}

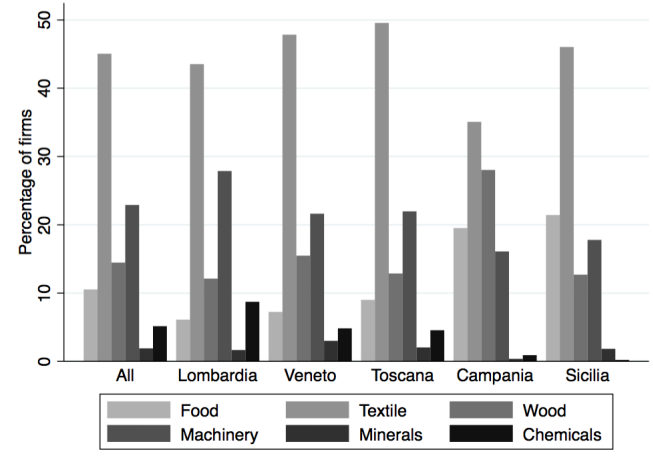

Panel A: By Region

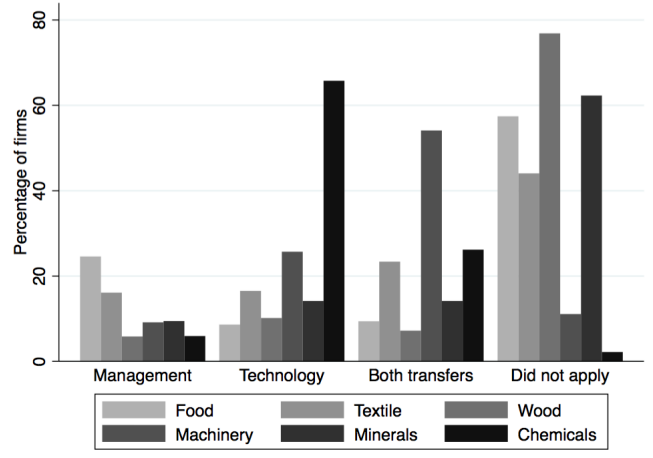

Panel B: By U.S. Transfer Chosen

\begin{abstract}
Notes. Distribution of 6,065 firms eligible to apply for the Productivity Program by manufacturing industry in 1951. Panel A presents the distribution separately for the five pilot regions; Panel B presents the distribution separately for the U.S. transfer chosen by firms. Industries are defined according to the 1951 National Institute for Statistics (ISTAT) classification. Food includes food, beverage, and tobacco industries; Textile includes textile, wearing apparel, and leather industries; Wood includes wood and wood products (including furniture); Machinery includes fabricated metal products, machinery, and equipment; Minerals includes nonmetallic mineral products, except products of petroleum, and coal; Chemicals includes manufacture of chemicals and chemical, petroleum, coal, rubber, and plastic products.
\end{abstract}

Figure A.1. : Distribution of Eligible Firms by Industries, 1951 

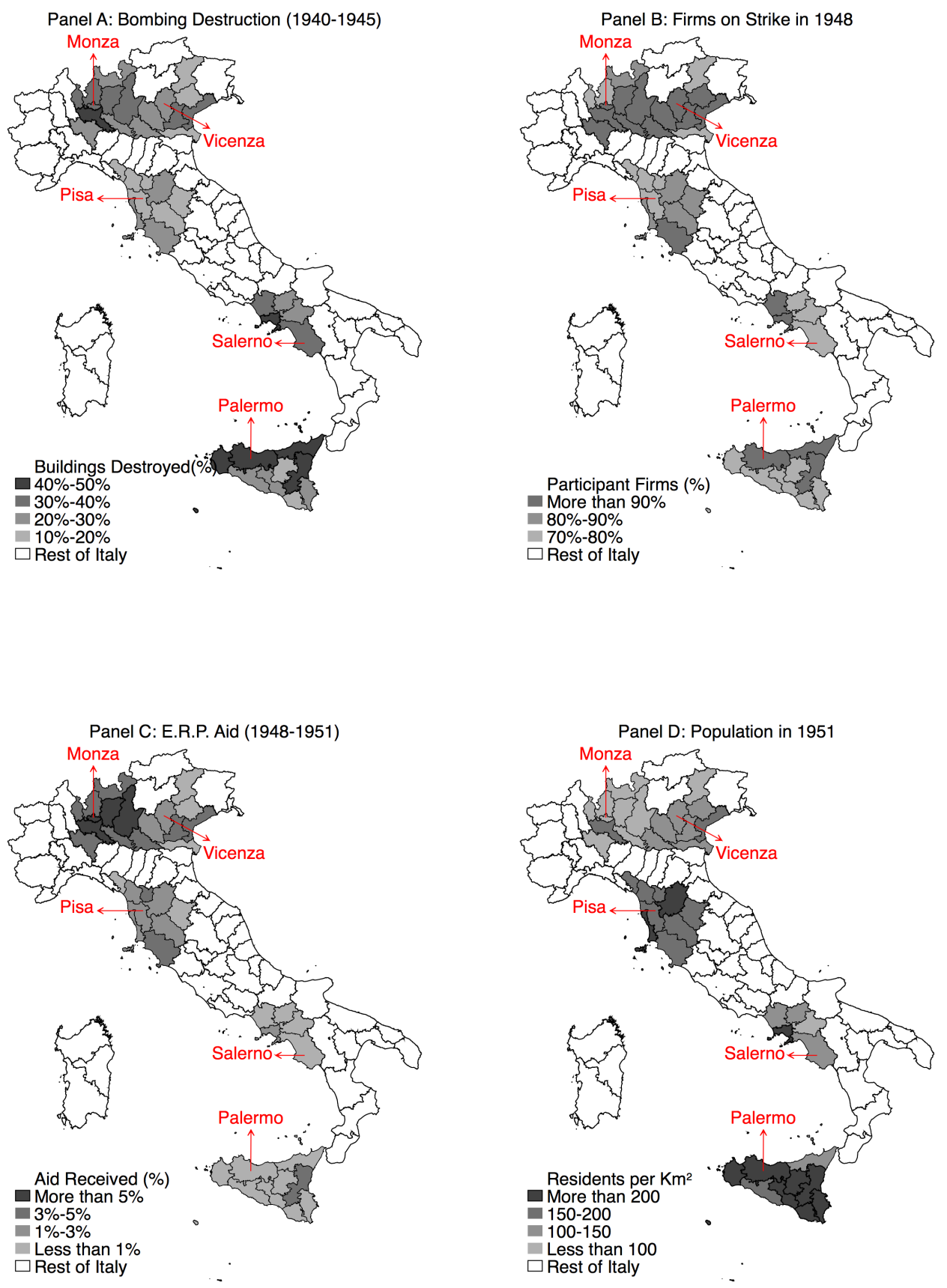

Figure A.2. : Treated and Comparison Provinces 

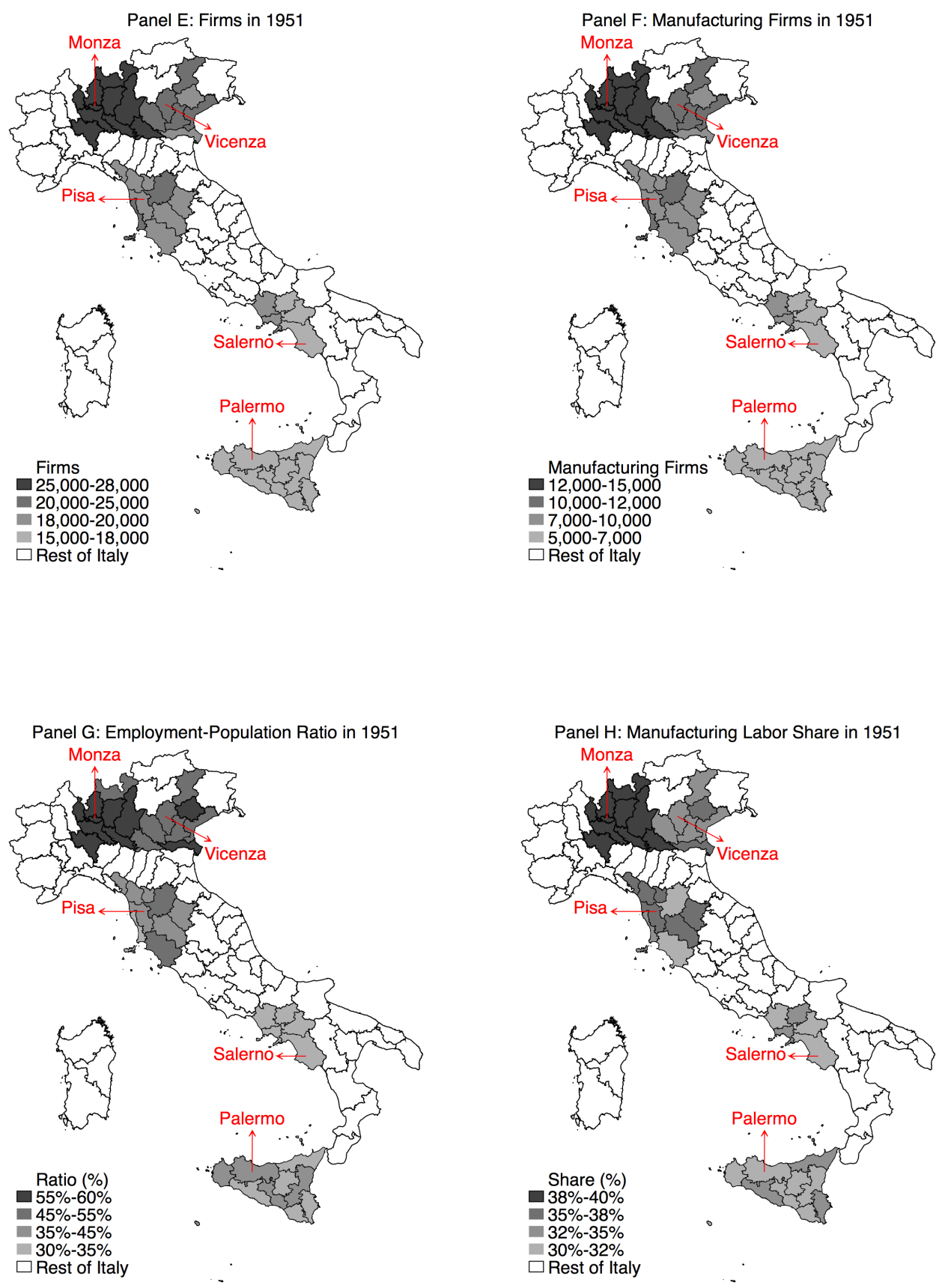

Figure A.2. : Treated and Comparison Provinces (cont.) 


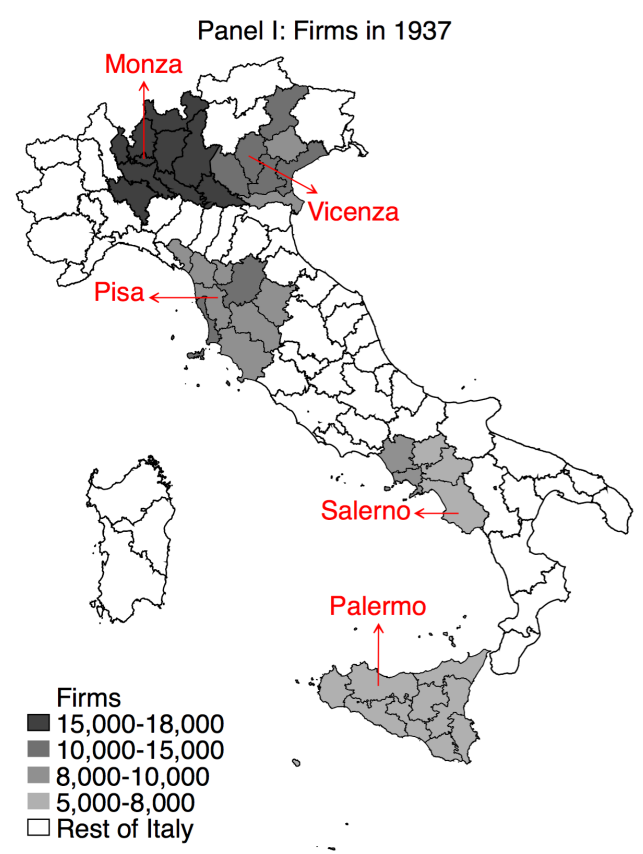

Panel J: Manufacturing Firms in 1937

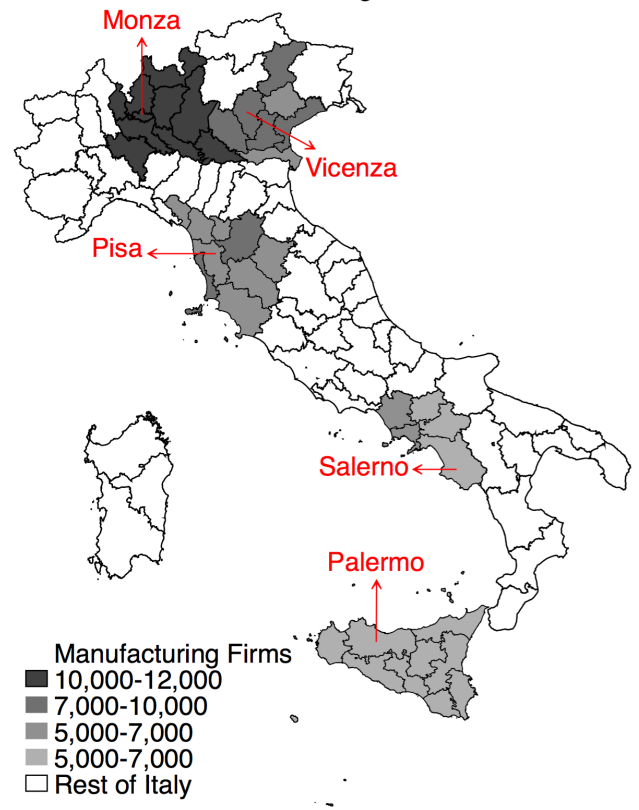

Figure A.2. : Treated and Comparison Provinces (cont.) 


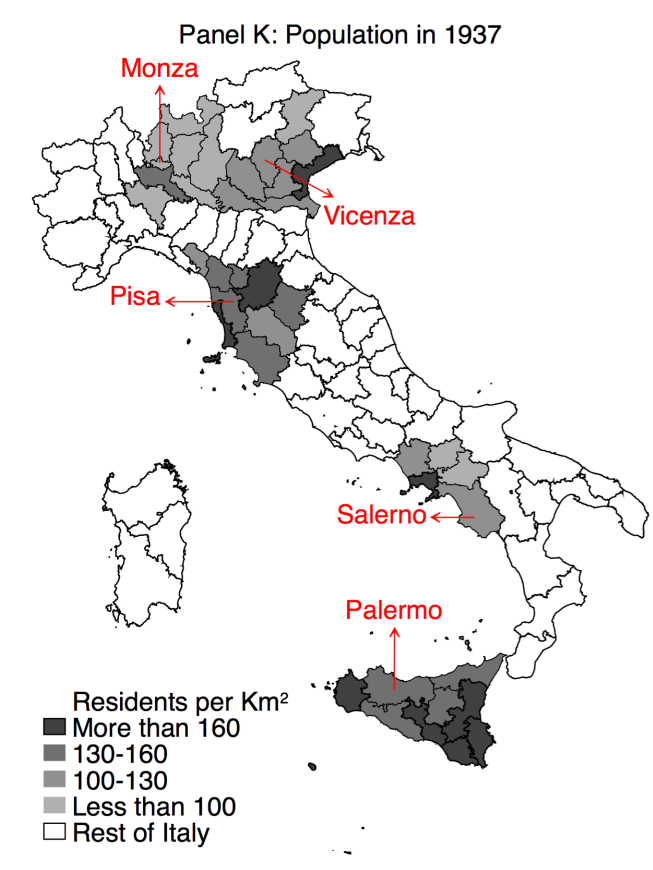

Notes. Maps showing percentage of buildings in a province destroyed by bombing between 1940 and 1945 (Panel A), percentage of firms involved in 1948 communist strikes (Panel B), E.R.P. aid received between 1948 and 1951 as fraction of total aid received by Italy (Panel C), population in 1951 and in 1937 (Panels D and K), total number of firms (Panels E and I), manufacturing firms (Panels F and J), employment-population ratio (Panel F), and labor share (Panel G). Data are provided at the province level. Data for Panels A, B and C were collected from the Archivio Storico dello Stato (Rome-Italy), fondo CIR, busta 39, accessed on January 12, 2013. Data for population are from the Italian Population Censuses of 1951 and 1936. The remaining data are from the Italian Industrial Censuses of 1951 and 1937.

Figure A.2. : Treated and Comparison Provinces (cont.) 


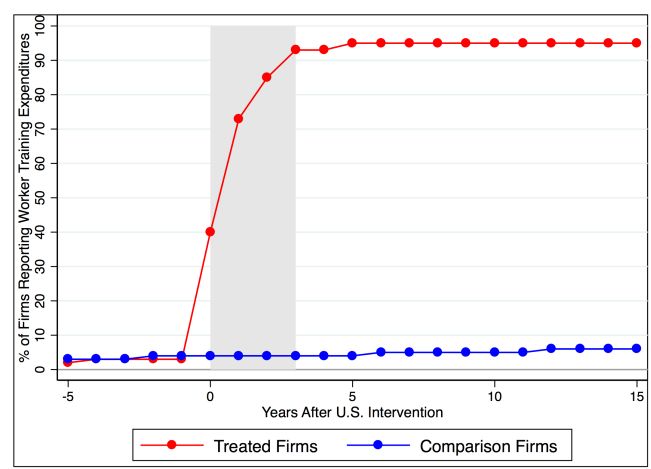

Panel A: Management Worker Training

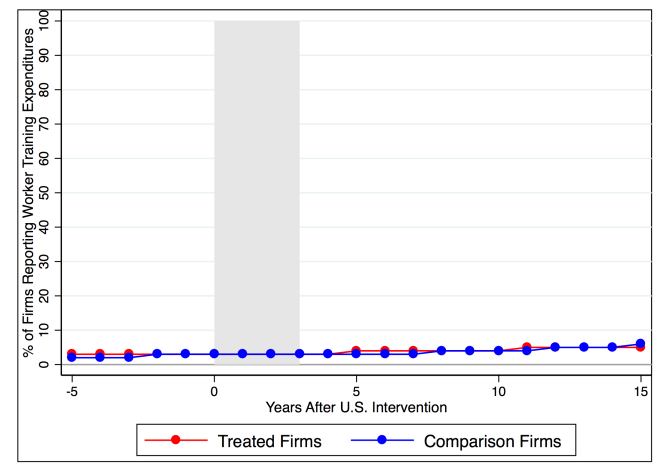

Panel C: Technology Worker Training

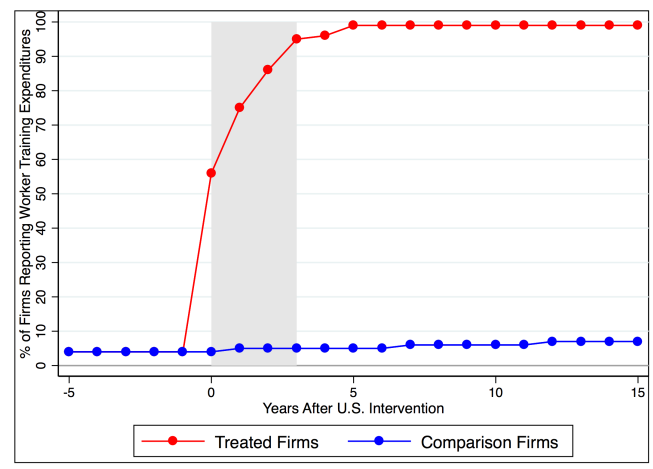

Panel E: Combined Worker Training

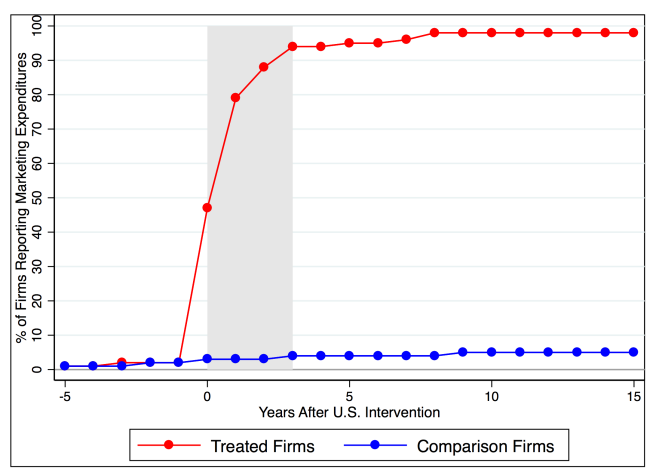

Panel B: Management Marketing

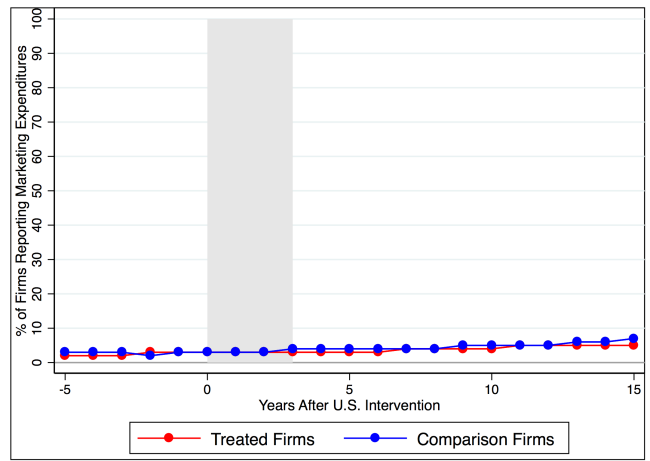

Panel D: Technology Marketing

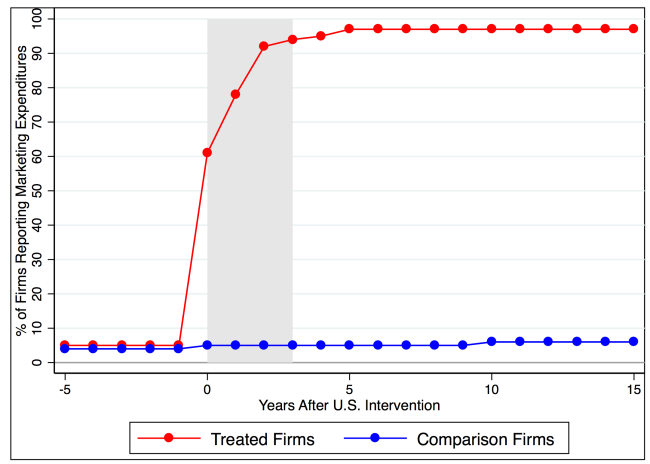

Panel F: Combined Marketing

Notes. Percentage of firms that were reporting expenditures for worker training (Panels A, C, and E) and marketing in their balance sheets (Panels B, D, and F) for 538 firms that applied for management transfer (Panels A-B), 748 firms that applied for technology transfer (Panels C-D), and 1,082 firms that applied for the combined management and technology transfers (Panels E-F). Data are provided at the firm level. The U.S. intervention year is normalized to zero, and the gray shaded area corresponds to the three-year follow-up period.

Figure A.3. : Effects of the Productivity Program on Managerial Practices Adoption 
Table A.1-: Summary Statistics by Pilot Regions, 1951

\begin{tabular}{llllll}
\hline \hline & \multicolumn{5}{c}{ All Eligible firms $(\mathrm{N}=6,065)$} \\
\cline { 2 - 6 } & Lombardia & Veneto & Toscana & Campania & Sicilia \\
& $(1)$ & $(2)$ & $(3)$ & $(4)$ & $(5)$ \\
\cline { 2 - 6 } Plants per firm & 1.54 & 1.23 & 1.24 & 1.13 & 1.17 \\
Employees per firm & 55.65 & 46.87 & 43.47 & 37.89 & 39.78 \\
Current assets (k USD) & $1,873.49$ & $1,546.73$ & $1,567.89$ & $1,289.28$ & $1,432.55$ \\
Annual sales (k USD) & $1,278.90$ & $1,345.98$ & 978.90 & 357.21 & 392.26 \\
Value added (k USD) & 567.88 & 489.76 & 398.58 & 409.32 & 459.10 \\
Age & 12.58 & 13.57 & 11.69 & 10.38 & 12.50 \\
Productivity (log TFPR) & 2.71 & 2.44 & 2.39 & 2.25 & 2.21 \\
Export & 0.15 & 0.13 & 0.12 & 0.09 & 0.12 \\
Family-managed & 0.36 & 0.41 & 0.48 & 0.48 & 0.54 \\
Submit application & 0.63 & 0.61 & 0.65 & 0.47 & 0.47 \\
Management & 0.13 & 0.16 & 0.16 & 0.11 & 0.07 \\
Technology & 0.19 & 0.19 & 0.26 & 0.16 & 0.19 \\
Combined management & 0.31 & 0.27 & 0.28 & 0.20 & 0.12 \\
and technology transfers & & & & & 963 \\
\hline Observations & 2,301 & 1,207 & 1,038 & 556 & \\
\hline \hline
\end{tabular}

Notes. Summary statistics for the 6,065 firms eligible to apply for the Productivity Program in 1951, separately by pilot regions. Data are provided at the firm level. Column 1 reports the mean for 2,301 eligible firms in Lombardia, column 2 for 1,207 firms in Veneto, column 3 for 1,038 firms in Toscana, column 4 for 556 firms in Campania, and column 5 for 963 firms in Sicilia. Plants per firm reports the number of plants per firm; Employees per firm reports the number of employees per firm; Current assets, Annual sales, and Value added are in 2010 USD, reevaluated from 1951 to 2010 values at 1 lira $=30.884$ euros and exchanged at 0.780 euro=USD 1; Productivity (log TFPR) is the logarithm of firm productivity, estimated using the Ackerberg, Caves and Frazer (2006) method; Export, Familymanaged, Submit application, Management, Technology, Combined management and technology transfers are indicator variables that equal one if, respectively, a firm exports, is family-managed, had submitted an application for the Productivity Program, and chose the management transfer, the technology transfer, or the combined management and technology transfers. 
Table A.2-: Pre-Productivity Program Differences Treated and Comparison Provinces

\begin{tabular}{|c|c|c|c|c|c|c|c|c|}
\hline & $\begin{array}{l}\text { Total Firms } \\
\text { (1) }\end{array}$ & $\begin{array}{l}\text { Mfg. Firms } \\
(2)\end{array}$ & $\begin{array}{l}\text { Population } \\
(3)\end{array}$ & $\begin{array}{l}\text { Empl./Pop. } \\
\text { (4) }\end{array}$ & $\begin{array}{l}\text { Labor Share } \\
(5)\end{array}$ & $\begin{array}{l}\text { Damage } \\
(6)\end{array}$ & $\begin{array}{l}\text { Strikes } \\
(7)\end{array}$ & $\begin{array}{l}\text { Aid } \\
(8)\end{array}$ \\
\hline \multicolumn{9}{|l|}{ Panel A. 1951 Census } \\
\hline \multicolumn{9}{|l|}{ Pilot region indicators } \\
\hline \multirow[t]{2}{*}{ Lombardia } & 27.000 & 13.667 & $4,445.889$ & 58.667 & 46.222 & 88.889 & 86.667 & 4.333 \\
\hline & $(0.591)$ & $(0.488)$ & $(1,427.536)$ & $(0.875)$ & $(0.959)$ & $(3.176)$ & $(2.132)$ & $(0.275)$ \\
\hline \multirow[t]{2}{*}{ Veneto } & 21.000 & 11.000 & $6,279.333$ & 55.167 & 35.167 & 80.000 & 83.500 & 2.833 \\
\hline & $(0.724)$ & $(0.597)$ & $(1,748.367)$ & $(1.072)$ & $(1.175)$ & $(3.890)$ & $(2.611)$ & $(0.337)$ \\
\hline \multirow[t]{2}{*}{ Toscana } & 19.500 & 9.500 & $11,300.625$ & 48.000 & 35.500 & 75.625 & 81.500 & 3.000 \\
\hline & $(0.627)$ & $(0.517)$ & $(1,514.130)$ & $(0.928)$ & $(1.017)$ & $(3.369)$ & $(2.261)$ & $(0.292)$ \\
\hline \multirow[t]{2}{*}{ Campania } & 16.250 & 6.250 & $9,571.000$ & 41.000 & 31.000 & 87.500 & 85.000 & 1.750 \\
\hline & $(0.886)$ & $(0.732)$ & $(2,141.304)$ & $(1.313)$ & $(1.439)$ & $(4.764)$ & $(3.198)$ & $(0.413)$ \\
\hline \multirow[t]{2}{*}{ Sicilia } & 14.250 & 4.250 & $14,328.500$ & 45.000 & 30.000 & 83.750 & 80.625 & 1.875 \\
\hline & $(0.627)$ & $(0.517)$ & $(1,514.130)$ & $(0.928)$ & $(1.017)$ & $(3.369)$ & $(2.261)$ & $(0.292)$ \\
\hline \multicolumn{9}{|c|}{ Treatment province indicators } \\
\hline \multirow[t]{2}{*}{ Monza } & 2.000 & 0.333 & -445.889 & 1.333 & 3.778 & 1.111 & 3.333 & 0.667 \\
\hline & $(1.868)$ & $(1.543)$ & $(4,514.264)$ & $(2.768)$ & $(3.033)$ & $(10.043)$ & $(6.742)$ & $(0.870)$ \\
\hline \multirow[t]{2}{*}{ Vicenza } & 2.000 & 1.000 & $-1,295.333$ & -0.167 & -0.167 & -0.000 & 3.500 & 0.167 \\
\hline & $(1.914)$ & $(1.581)$ & $(4,625.744)$ & $(2.836)$ & $(3.108)$ & $(10.291)$ & $(6.909)$ & $(0.892)$ \\
\hline \multirow[t]{2}{*}{ Pisa } & 1.500 & 0.500 & $-2,089.625$ & -1.000 & 1.500 & -0.625 & -1.500 & 0.000 \\
\hline & $(1.880)$ & $(1.552)$ & $(4,542.391)$ & $(2.785)$ & $(3.052)$ & $(10.106)$ & $(6.784)$ & $(0.876)$ \\
\hline \multirow[t]{2}{*}{ Salerno } & -0.250 & -0.250 & $-4,241.000$ & -1.000 & -1.000 & 2.500 & -5.000 & 0.250 \\
\hline & $(1.982)$ & $(1.636)$ & $(4,788.100)$ & $(2.936)$ & $(3.217)$ & $(10.652)$ & $(7.151)$ & (0.923) \\
\hline \multirow[t]{2}{*}{ Palermo } & -0.250 & -0.250 & $-1,059.500$ & 3.000 & 1.000 & 11.250 & 9.375 & -0.875 \\
\hline & $(1.880)$ & $(1.552)$ & $(4,542.391)$ & $(2.785)$ & $(3.052)$ & $(10.106)$ & $(6.784)$ & $(0.876)$ \\
\hline Number of observations & 40 & 40 & 40 & 40 & 40 & 40 & 40 & 40 \\
\hline
\end{tabular}


Table A.2-: Continued

\begin{tabular}{|c|c|c|c|}
\hline & $\begin{array}{l}\text { Total Firms } \\
\text { (1) }\end{array}$ & $\begin{array}{l}\text { Mfg. Firms } \\
(2)\end{array}$ & $\begin{array}{l}\text { Population } \\
(3)\end{array}$ \\
\hline \multicolumn{4}{|l|}{ Panel B. 1937 Census } \\
\hline \multicolumn{4}{|l|}{ Pilot region indicators } \\
\hline \multirow[t]{2}{*}{ Lombardia } & 17.000 & 12.222 & $4,174.333$ \\
\hline & $(0.495)$ & $(0.382)$ & $(1,272.256)$ \\
\hline \multirow[t]{2}{*}{ Veneto } & 9.333 & 7.000 & $6,908.167$ \\
\hline & $(0.606)$ & $(0.468)$ & $(1,558.189)$ \\
\hline \multirow[t]{2}{*}{ Toscana } & 7.500 & 6.000 & $10,068.625$ \\
\hline & $(0.525)$ & $(0.405)$ & $(1,349.432)$ \\
\hline \multirow[t]{2}{*}{ Campania } & 6.250 & 4.000 & $8,152.250$ \\
\hline & $(0.742)$ & $(0.573)$ & $(1,908.385)$ \\
\hline \multirow[t]{2}{*}{ Sicilia } & 5.000 & 3.375 & $13,477.500$ \\
\hline & $(0.525)$ & $(0.405)$ & $(1,349.432)$ \\
\hline \multicolumn{4}{|c|}{ Treatment province indicators } \\
\hline \multirow[t]{2}{*}{ Monza } & -0.000 & 1.778 & -774.333 \\
\hline & $(1.564)$ & $(1.208)$ & $(4,023.228)$ \\
\hline \multirow[t]{2}{*}{ Vicenza } & 1.667 & 1.000 & -957.167 \\
\hline & $(1.603)$ & $(1.238)$ & $(4,122.582)$ \\
\hline \multirow[t]{2}{*}{ Pisa } & 0.500 & 1.000 & $-1,083.625$ \\
\hline & $(1.574)$ & $(1.216)$ & $(4,048.295)$ \\
\hline \multirow[t]{2}{*}{ Salerno } & -0.250 & -1.000 & $-3,660.250$ \\
\hline & $(1.659)$ & $(1.282)$ & $(4,267.278)$ \\
\hline \multirow[t]{2}{*}{ Palermo } & -1.000 & -0.375 & $-1,909.500$ \\
\hline & $(1.574)$ & $(1.216)$ & $(4,048.295)$ \\
\hline Number of observations & 40 & 40 & 40 \\
\hline
\end{tabular}

Notes. OLS regressions predicting province-level outcomes in 1951 (Panel A) and 1937 (Panel B). Lombardia, Veneto, Toscana, Campania, and Sicilia are indicator variables for pilot regions. Monza, Vicenza, Pisa, Salerno, and Palermo are indicator variables for treatment provinces. The dependent variables are total number of firms (column 1), manufacturing firms (column 2), population (column 3), employmentpopulation ratio (4), labor share (5), percentage of buildings in a province destroyed by bombing between 1940 and 1945 (column 6), percentage of firms involved in 1948 communist strikes (column 7), and E.R.P. aid received between 1948 and 1951 as a fraction of total aid received by Italy (column 8). Data are provided at the province level. Data for columns 6-8 were collected from the Archivio Storico A4 dello Stato (Rome-Italy), fondo CIR, busta 39, accessed on January 12, 2013. Data for population are from the Italian Population Censuses of 1951 and 1936. The remaining data are from the Italian Industrial Censuses of 1951 and 1937. 
Table A.3-: Pre-Productivity Program Differences in Growth Rates between Treated and Comparison Provinces, 1937-1951

\begin{tabular}{|c|c|c|c|}
\hline & $\begin{array}{c}\text { Growth Rate } \\
\text { Total Firms } \\
\text { (1) }\end{array}$ & $\begin{array}{l}\text { Growth Rate } \\
\text { Mfg. Firms } \\
\text { (2) }\end{array}$ & $\begin{array}{c}\text { Growth Rate } \\
\text { Population } \\
(3)\end{array}$ \\
\hline \multicolumn{4}{|l|}{ Pilot region indicators } \\
\hline \multirow[t]{2}{*}{ Lombardia } & 4.256 & 0.884 & 0.794 \\
\hline & $(0.819)$ & $(0.593)$ & $(0.657)$ \\
\hline \multirow[t]{2}{*}{ Veneto } & 9.411 & 4.174 & 0.266 \\
\hline & $(1.003)$ & $(0.726)$ & $(0.805)$ \\
\hline \multirow[t]{2}{*}{ Toscana } & 11.651 & 4.498 & 0.980 \\
\hline & $(0.868)$ & $(0.629)$ & $(0.697)$ \\
\hline \multirow[t]{2}{*}{ Campania } & 11.650 & 4.137 & 1.053 \\
\hline & $(1.228)$ & $(0.889)$ & $(0.986)$ \\
\hline \multirow[t]{2}{*}{ Sicilia } & 13.363 & 1.637 & 0.398 \\
\hline & $(0.868)$ & $(0.629)$ & $(0.697)$ \\
\hline \multicolumn{4}{|c|}{ Treatment province indicators } \\
\hline \multirow[t]{2}{*}{ Monza } & 0.786 & -0.884 & 0.466 \\
\hline & $(2.589)$ & $(1.875)$ & $(2.078)$ \\
\hline \multirow[t]{2}{*}{ Vicenza } & -1.619 & -0.602 & -1.427 \\
\hline & $(2.653)$ & $(1.921)$ & $(2.130)$ \\
\hline \multirow[t]{2}{*}{ Pisa } & -0.044 & -1.437 & -0.800 \\
\hline & $(2.605)$ & $(1.887)$ & $(2.091)$ \\
\hline \multirow[t]{2}{*}{ Salerno } & 0.255 & 3.006 & 0.280 \\
\hline & $(2.746)$ & $(1.989)$ & $(2.205)$ \\
\hline \multirow[t]{2}{*}{ Palermo } & 4.494 & 0.744 & 0.653 \\
\hline & $(2.605)$ & $(1.887)$ & $(2.091)$ \\
\hline Number of observations & 40 & 40 & 40 \\
\hline
\end{tabular}

Notes. OLS regressions predicting province-level growth rates between 1937 and 1951. Lombardia, Veneto, Toscana, Campania, and Sicilia are indicator variables for pilot regions. Monza, Vicenza, Pisa, Salerno, and Palermo are indicator variables for treatment provinces. The dependent variables are the growth rate of total number of firms (column 1), manufacturing firms (column 2), and population (column 3). Data for population are from the Italian Population Censuses of 1951 and 1936. The remaining data are from the Italian Industrial Censuses of 1951 and 1937. 
Table A.4-: ANOVA Test for Treated and Comparison Provinces

\begin{tabular}{|c|c|c|c|c|c|c|}
\hline & $\begin{array}{c}\text { Treated }=\text { Comparison } \\
(1)\end{array}$ & $\begin{array}{c}\text { Lombardia }=\text { Monza } \\
\qquad(2)\end{array}$ & $\begin{array}{c}\text { Veneto }=\text { Vicenza } \\
(3)\end{array}$ & $\begin{array}{c}\text { Toscana }=\text { Pisa } \\
(4)\end{array}$ & $\begin{array}{c}\text { Campania }=\text { Salerno } \\
(5)\end{array}$ & $\begin{array}{c}\text { Sicilia }=\text { Palermo } \\
(6)\end{array}$ \\
\hline \multirow[t]{2}{*}{ Total firms in 1951} & 0.04 & 0.76 & 2.14 & 0.78 & 0.05 & 0.01 \\
\hline & $(0.844)$ & $(0.409)$ & $(0.203)$ & $(0.407)$ & $(0.830)$ & $(0.908)$ \\
\hline \multirow[t]{2}{*}{ Manufacturing firms in 1951} & 0.00 & 0.10 & 0.54 & 0.09 & 0.05 & 0.01 \\
\hline & $(0.975)$ & $(0.760)$ & $(0.497)$ & $(0.777)$ & $(0.830)$ & $(0.908)$ \\
\hline \multirow[t]{2}{*}{ Population in 1951} & 0.49 & 0.03 & 0.73 & 0.32 & 0.16 & 0.05 \\
\hline & $(0.487)$ & $(0.868)$ & $(0.433)$ & $(0.592)$ & $(0.714)$ & $(0.829)$ \\
\hline \multirow[t]{2}{*}{ Employment/Population in 1951} & 0.02 & 0.21 & 0.00 & 0.19 & 0.24 & 0.76 \\
\hline & $(0.884)$ & $(0.661)$ & $(0.951)$ & $(0.673)$ & $(0.658)$ & $(0.413)$ \\
\hline \multirow[t]{2}{*}{ Labor share in 1951} & 0.00 & 1.40 & 0.00 & 0.17 & 0.24 & 0.12 \\
\hline & $(0.959)$ & $(0.271)$ & $(0.951)$ & $(0.692)$ & $(0.658)$ & $(0.744)$ \\
\hline \multirow[t]{2}{*}{ Damage } & 0.39 & 0.05 & 0.000 & 0.01 & 0.05 & 0.50 \\
\hline & $(0.538)$ & $(0.834)$ & $(1.000)$ & $(0.919)$ & $(0.830)$ & $(0.504)$ \\
\hline \multirow[t]{2}{*}{ Strikes } & 0.45 & 0.32 & 0.18 & 0.05 & 0.60 & 1.70 \\
\hline & $(0.507)$ & $(0.587)$ & $(0.693)$ & $(0.822)$ & $(0.495)$ & $(0.234)$ \\
\hline \multirow[t]{2}{*}{ Aid } & 0.04 & 1.60 & 0.02 & 0.00 & 0.05 & 0.69 \\
\hline & $(0.852)$ & $(0.242)$ & $(0.881)$ & $(1.000)$ & $(0.830)$ & $(0.433)$ \\
\hline \multirow[t]{2}{*}{ Total firms in 1937} & 0.02 & 0.00 & 0.69 & 0.26 & 0.05 & 3.11 \\
\hline & $(0.884)$ & $(1.000)$ & $(0.445)$ & $(0.626)$ & $(0.830)$ & $(0.121)$ \\
\hline \multirow[t]{2}{*}{ Manufacturing firms in 1937} & 0.00 & 1.97 & 2.14 & 0.62 & 1.20 & 0.06 \\
\hline & $(0.974)$ & $(0.198)$ & $(0.203)$ & $(0.456)$ & $(0.353)$ & $(0.810)$ \\
\hline \multirow[t]{2}{*}{ Population in 1937} & 0.54 & 0.12 & 0.05 & 0.09 & 0.18 & 0.34 \\
\hline & $(0.468)$ & $(0.734)$ & $(0.833)$ & $(0.767)$ & $(0.700)$ & $(0.576)$ \\
\hline
\end{tabular}

Notes. ANOVA test for mean equality among treated and comparison provinces (column 1), and each pilot region and its treatment province (columns $2-6)$. For each variable, the first row reports the $F$-statistics and the second row the $p$-value. The variables are total number of firms, manufacturing firms, population, employment-population ratio, labor share, percentage of buildings in a province destroyed by bombing between 1940 and 1945 , percentage of firms involved in 1948 communist strikes, and E.R.P. aid received between 1948 and 1951 as a fraction of total aid received by Italy. Data are provided at the province level. 
Table A.5-: Pre-Productivity Program Differences in Time Trends between Treated and Comparison Provinces, $1946-1951$

\begin{tabular}{|c|c|c|c|c|c|c|c|c|c|c|}
\hline & \multicolumn{2}{|c|}{ Log Employment } & \multicolumn{2}{|c|}{ Log Assets } & \multicolumn{2}{|c|}{ Log Sales } & \multicolumn{2}{|c|}{ Log Value Added } & \multicolumn{2}{|c|}{ Log TFPR } \\
\hline & $(1)$ & $(2)$ & $(3)$ & $(4)$ & $(5)$ & $(6)$ & $(7)$ & $(8)$ & $(9)$ & $(10)$ \\
\hline \multicolumn{11}{|l|}{ Panel A. Management } \\
\hline \multirow[t]{2}{*}{ Time trend } & 0.031 & 0.027 & 0.033 & 0.038 & 0.043 & 0.036 & 0.029 & 0.026 & 0.016 & 0.014 \\
\hline & $(0.015)$ & $(0.013)$ & $(0.019)$ & $(0.018)$ & $(0.011)$ & $(0.013)$ & $(0.006)$ & $(0.005)$ & $(0.003)$ & $(0.003)$ \\
\hline \multirow[t]{2}{*}{ Time trend . Treatment Province } & 0.013 & 0.011 & -0.012 & -0.014 & 0.012 & 0.009 & 0.019 & 0.010 & 0.014 & 0.010 \\
\hline & $(0.013)$ & $(0.015)$ & $(0.013)$ & $(0.017)$ & $(0.017)$ & $(0.015)$ & $(0.025)$ & $(0.018)$ & $(0.015)$ & $(0.012)$ \\
\hline \multirow[t]{2}{*}{ Treatment Province } & 0.011 & 0.014 & -0.007 & -0.009 & -0.009 & -0.012 & -0.008 & -0.006 & 0.020 & 0.018 \\
\hline & $(0.013)$ & $(0.012)$ & $(0.009)$ & $(0.014)$ & $(0.014)$ & $(0.016)$ & $(0.011)$ & $(0.009)$ & $(0.026)$ & $(0.022)$ \\
\hline Observations & 3,141 & 3,141 & 3,141 & 3,141 & 3,141 & 3,141 & 3,141 & 3,141 & 3,141 & 3,141 \\
\hline \multicolumn{11}{|l|}{ Panel B. Technology } \\
\hline \multirow[t]{2}{*}{ Time trend } & 0.039 & 0.035 & 0.029 & 0.026 & 0.055 & 0.054 & 0.041 & 0.037 & 0.015 & 0.011 \\
\hline & $(0.017)$ & $(0.013)$ & $(0.013)$ & $(0.011)$ & $(0.033)$ & $(0.032)$ & $(0.013)$ & $(0.014)$ & $(0.004)$ & $(0.003)$ \\
\hline \multirow[t]{2}{*}{ Time trend $\cdot$ Treatment Province } & -0.006 & -0.003 & 0.010 & 0.008 & 0.006 & 0.005 & 0.009 & 0.007 & -0.005 & -0.005 \\
\hline & $(0.010)$ & $(0.009)$ & $(0.014)$ & $(0.012)$ & $(0.008)$ & $(0.008)$ & $(0.010)$ & $(0.007)$ & $(0.008)$ & $(0.010)$ \\
\hline \multirow[t]{2}{*}{ Treatment Province } & 0.014 & 0.016 & 0.015 & 0.010 & -0.013 & -0.012 & 0.011 & 0.009 & -0.006 & -0.003 \\
\hline & $(0.021)$ & $(0.019)$ & $(0.023)$ & $(0.019)$ & $(0.019)$ & $(0.015)$ & $(0.010)$ & $(0.014)$ & $(0.009)$ & $(0.007)$ \\
\hline Observations & 4,678 & 4,678 & 4,678 & 4,678 & 4,678 & 4,678 & 4,678 & 4,678 & 4,678 & 4,678 \\
\hline \multicolumn{11}{|l|}{ Panel C. Combined } \\
\hline \multirow[t]{2}{*}{ Time trend } & 0.046 & 0.041 & 0.038 & 0.035 & 0.045 & 0.041 & 0.049 & 0.048 & 0.018 & 0.016 \\
\hline & $(0.009)$ & $(0.008)$ & $(0.012)$ & $(0.010)$ & $(0.009)$ & $(0.008)$ & $(0.013)$ & $(0.011)$ & $(0.005)$ & $(0.004)$ \\
\hline \multirow[t]{2}{*}{ Time trend . Treatment Province } & 0.008 & 0.010 & -0.021 & -0.010 & -0.007 & -0.008 & 0.004 & 0.006 & -0.008 & -0.008 \\
\hline & $(0.011)$ & $(0.013)$ & $(0.029)$ & $(0.025)$ & $(0.011)$ & $(0.010)$ & $(0.007)$ & $(0.012)$ & $(0.015)$ & $(0.011)$ \\
\hline \multirow[t]{2}{*}{ Treatment Province } & -0.017 & -0.015 & 0.005 & 0.003 & 0.011 & 0.014 & -0.009 & -0.014 & 0.017 & 0.014 \\
\hline & $(0.022)$ & $(0.019)$ & $(0.006)$ & $(0.008)$ & $(0.013)$ & $(0.021)$ & $(0.015)$ & $(0.015)$ & $(0.020)$ & $(0.019)$ \\
\hline Observations & 6,238 & 6,238 & 6,238 & 6,238 & 6,238 & 6,238 & 6,238 & 6,238 & 6,238 & 6,238 \\
\hline Pilot region & Yes & Yes & Yes & Yes & Yes & Yes & Yes & Yes & Yes & Yes \\
\hline Pilot region $\mathrm{x}$ time $\mathrm{FE}$ & No & Yes & No & Yes & No & Yes & No & Yes & No & Yes \\
\hline
\end{tabular}

Notes. OLS regressions predicting outcomes in the pre-Productivity Program period for 804 firms that applied for management transfer (Panel A), 1,178 firms that applied for technology transfer (Panel B), and 1,612 firms that applied for the combined management and technology transfers (Panel C). Thirty firms whose applications were rejected are excluded. Data are provided at the firm level. Outcomes are allowed to vary according to a linear time (year) trend that differs for treatment provinces. Excluded year is 1946. Standard errors are block-bootstrapped at the province level with 200 replications. All the dependent variables are expressed in logs. Employment is the number of employees per firm; Assets, Sales, and Value Added are in 2010 USD, reevaluated from 1951 to 2010 values at 1 lira $=30.884$ euros and exchanged at 0.780 euro=USD 1 ; TFPR is the logarithm of total factor productivity revenue, estimated using the Ackerberg, Caves and Frazer (2006) method. 
Table A.6-: Pre-Productivity Program Differences in Region Time Trends, $1946-1951$

\begin{tabular}{|c|c|c|c|c|c|}
\hline & $\begin{array}{l}\text { Log Empl. } \\
\text { (1) }\end{array}$ & $\begin{array}{l}\text { Log Assets } \\
(2)\end{array}$ & $\begin{array}{l}\text { Log Sales } \\
(3)\end{array}$ & $\begin{array}{l}\text { Log Value Added } \\
(4)\end{array}$ & $\begin{array}{l}\text { Log TFPR } \\
\text { (5) }\end{array}$ \\
\hline \multicolumn{6}{|l|}{$\begin{array}{l}\text { Panel A. Management } \\
\text { Pilot region indicators }\end{array}$} \\
\hline Time Trend $\cdot$ Lombardia & $\begin{array}{l}0.043 \\
(0.019)\end{array}$ & $\begin{array}{l}0.048 \\
(0.025)\end{array}$ & $\begin{array}{l}0.052 \\
(0.030)\end{array}$ & $\begin{array}{l}0.034 \\
(0.020)\end{array}$ & $\begin{array}{l}0.020 \\
(0.006)\end{array}$ \\
\hline Time Trend $\cdot$ Veneto & $\begin{array}{l}0.034 \\
(0.015)\end{array}$ & $\begin{array}{l}0.040 \\
(0.019)\end{array}$ & $\begin{array}{l}0.046 \\
(0.018)\end{array}$ & $\begin{array}{l}0.027 \\
(0.012)\end{array}$ & $\begin{array}{l}0.017 \\
(0.009)\end{array}$ \\
\hline Time Trend $\cdot$ Toscana & $\begin{array}{l}0.030 \\
(0.014)\end{array}$ & $\begin{array}{l}0.035 \\
(0.017)\end{array}$ & $\begin{array}{l}0.041 \\
(0.022)\end{array}$ & $\begin{array}{l}0.025 \\
(0.012)\end{array}$ & $\begin{array}{l}0.015 \\
(0.007)\end{array}$ \\
\hline Time Trend · Campania & $\begin{array}{l}0.020 \\
(0.009)\end{array}$ & $\begin{array}{l}0.012 \\
(0.006)\end{array}$ & $\begin{array}{l}0.028 \\
(0.014)\end{array}$ & $\begin{array}{l}0.016 \\
(0.007)\end{array}$ & $\begin{array}{l}0.010 \\
(0.006)\end{array}$ \\
\hline Time Trend $\cdot$ Sicilia & $\begin{array}{l}0.016 \\
(0.008)\end{array}$ & $\begin{array}{l}0.015 \\
(0.009)\end{array}$ & $\begin{array}{l}0.024 \\
(0.012)\end{array}$ & $\begin{array}{l}0.017 \\
(0.006)\end{array}$ & $\begin{array}{l}0.009 \\
(0.004)\end{array}$ \\
\hline \multicolumn{6}{|c|}{ Treatment province indicators } \\
\hline Time Trend $\cdot$ Monza & $\begin{array}{l}0.006 \\
(0.008)\end{array}$ & $\begin{array}{l}0.003 \\
(0.005)\end{array}$ & $\begin{array}{l}0.004 \\
(0.012)\end{array}$ & $\begin{array}{l}-0.003 \\
(0.007)\end{array}$ & $\begin{array}{l}-0.002 \\
(0.009)\end{array}$ \\
\hline Time Trend · Vicenza & $\begin{array}{l}0.002 \\
(0.004)\end{array}$ & $\begin{array}{l}-0.005 \\
(0.009)\end{array}$ & $\begin{array}{l}-0.002 \\
(0.004)\end{array}$ & $\begin{array}{l}0.001 \\
(0.001)\end{array}$ & $\begin{array}{l}0.003 \\
(0.002)\end{array}$ \\
\hline Time Trend $\cdot$ Pisa & $\begin{array}{l}-0.004 \\
(0.007)\end{array}$ & $\begin{array}{l}-0.006 \\
(0.011)\end{array}$ & $\begin{array}{l}0.003 \\
(0.005)\end{array}$ & $\begin{array}{l}0.005 \\
(0.004)\end{array}$ & $\begin{array}{l}0.002 \\
(0.003)\end{array}$ \\
\hline Time Trend $\cdot$ Salerno & $\begin{array}{l}0.004 \\
(0.003)\end{array}$ & $\begin{array}{l}0.008 \\
(0.009)\end{array}$ & $\begin{array}{l}-0.003 \\
(0.004)\end{array}$ & $\begin{array}{l}0.005 \\
(0.011)\end{array}$ & $\begin{array}{l}0.007 \\
(0.008)\end{array}$ \\
\hline Time Trend · Palermo & $\begin{array}{l}-0.001 \\
(0.003)\end{array}$ & $\begin{array}{l}0.004 \\
(0.006)\end{array}$ & $\begin{array}{l}0.005 \\
(0.005)\end{array}$ & $\begin{array}{l}-0.006 \\
(0.008)\end{array}$ & $\begin{array}{l}-0.007 \\
(0.006)\end{array}$ \\
\hline Observations & 3,141 & 3,141 & 3,141 & 3,141 & 3,141 \\
\hline$F$-statistic & 0.61 & 0.57 & 0.65 & 0.76 & 0.27 \\
\hline \multicolumn{6}{|l|}{$\begin{array}{l}\text { Panel B. Technology } \\
\quad \text { Pilot region indicators }\end{array}$} \\
\hline Time Trend $\cdot$ Lombardia & $\begin{array}{l}0.049 \\
(0.022)\end{array}$ & $\begin{array}{l}0.042 \\
(0.022)\end{array}$ & $\begin{array}{l}0.045 \\
(0.023)\end{array}$ & $\begin{array}{l}0.029 \\
(0.015)\end{array}$ & $\begin{array}{l}0.024 \\
(0.012)\end{array}$ \\
\hline Time Trend · Veneto & $\begin{array}{l}0.038 \\
(0.022)\end{array}$ & $\begin{array}{l}0.045 \\
(0.023)\end{array}$ & $\begin{array}{l}0.049 \\
(0.021)\end{array}$ & $\begin{array}{l}0.033 \\
(0.017)\end{array}$ & $\begin{array}{l}0.021 \\
(0.012)\end{array}$ \\
\hline Time Trend · Toscana & $\begin{array}{l}0.035 \\
(0.016)\end{array}$ & $\begin{array}{l}0.041 \\
(0.024)\end{array}$ & $\begin{array}{l}0.048 \\
(0.025)\end{array}$ & $\begin{array}{l}0.029 \\
(0.014)\end{array}$ & $\begin{array}{l}0.018 \\
(0.009)\end{array}$ \\
\hline Time Trend · Campania & $\begin{array}{l}0.023 \\
(0.012)\end{array}$ & $\begin{array}{l}0.017 \\
(0.008)\end{array}$ & $\begin{array}{l}0.031 \\
(0.016)\end{array}$ & $\begin{array}{l}0.023 \\
(0.010)\end{array}$ & $\begin{array}{l}0.015 \\
(0.008)\end{array}$ \\
\hline Time Trend $\cdot$ Sicilia & $\begin{array}{l}0.022 \\
(0.012)\end{array}$ & $\begin{array}{l}0.016 \\
(0.008)\end{array}$ & $\begin{array}{l}0.029 \\
(0.017)\end{array}$ & $\begin{array}{l}0.021 \\
(0.010)\end{array}$ & $\begin{array}{l}0.010 \\
(0.006)\end{array}$ \\
\hline \multicolumn{6}{|c|}{ Treatment province indicators } \\
\hline Time Trend $\cdot$ Monza & $\begin{array}{l}0.011 \\
(0.010)\end{array}$ & $\begin{array}{l}-0.012 \\
(0.013)\end{array}$ & $\begin{array}{l}0.01 \\
(0.013)\end{array}$ & $\begin{array}{l}0.005 \\
(0.005)\end{array}$ & $\begin{array}{l}0.004 \\
(0.003)\end{array}$ \\
\hline Time Trend $\cdot$ Vicenza & $\begin{array}{l}-0.003 \\
(0.005)\end{array}$ & $\begin{array}{l}0.007 \\
(0.008)\end{array}$ & $\begin{array}{l}-0.006 \\
(0.008)\end{array}$ & $\begin{array}{l}0.003 \\
(0.003)\end{array}$ & $\begin{array}{l}0.005 \\
(0.007)\end{array}$ \\
\hline Time Trend $\cdot$ Pisa & $\begin{array}{l}0.006 \\
(0.005)\end{array}$ & $\begin{array}{l}-0.012 \\
(0.014)\end{array}$ & $\begin{array}{l}0.008 \\
(0.009)\end{array}$ & $\begin{array}{l}-0.004 \\
(0.003)\end{array}$ & $\begin{array}{l}-0.001 \\
(0.002)\end{array}$ \\
\hline
\end{tabular}


Table A.6-: Continued

\begin{tabular}{|c|c|c|c|c|c|}
\hline & $\begin{array}{l}\text { Log Empl. } \\
(1)\end{array}$ & $\begin{array}{l}\text { Log Assets } \\
(2)\end{array}$ & $\begin{array}{l}\text { Log Sales } \\
(3)\end{array}$ & $\begin{array}{l}\text { Log Value Added } \\
(4)\end{array}$ & $\begin{array}{l}\text { Log TFPR } \\
(5)\end{array}$ \\
\hline Time Trend $\cdot$ Salerno & $\begin{array}{l}-0.005 \\
(0.005)\end{array}$ & $\begin{array}{l}-0.004 \\
(0.006)\end{array}$ & $\begin{array}{l}0.005 \\
(0.005)\end{array}$ & $\begin{array}{l}0.007 \\
(0.009)\end{array}$ & $\begin{array}{l}-0.010 \\
(0.010)\end{array}$ \\
\hline Time Trend · Palermo & $\begin{array}{l}0.012 \\
(0.015)\end{array}$ & $\begin{array}{l}-0.005 \\
(0.010)\end{array}$ & $\begin{array}{l}-0.007 \\
(0.011)\end{array}$ & $\begin{array}{l}0.001 \\
(0.002)\end{array}$ & $\begin{array}{l}-0.005 \\
(0.005)\end{array}$ \\
\hline $\begin{array}{l}\text { Observations } \\
F \text {-statistic }\end{array}$ & $\begin{array}{l}4,678 \\
0.89\end{array}$ & $\begin{array}{l}4,678 \\
0.45\end{array}$ & $\begin{array}{l}4,678 \\
0.74 \\
\end{array}$ & $\begin{array}{l}4,678 \\
0.58\end{array}$ & $\begin{array}{l}4,678 \\
0.64\end{array}$ \\
\hline \multicolumn{6}{|l|}{$\begin{array}{l}\text { Panel C. Combined } \\
\quad \text { Pilot region indicators }\end{array}$} \\
\hline Time Trend · Lombardia & $\begin{array}{l}0.054 \\
(0.025)\end{array}$ & $\begin{array}{l}0.047 \\
(0.026)\end{array}$ & $\begin{array}{l}0.053 \\
(0.021)\end{array}$ & $\begin{array}{l}0.043 \\
(0.018)\end{array}$ & $\begin{array}{l}0.029 \\
(0.017)\end{array}$ \\
\hline Time Trend · Veneto & $\begin{array}{l}0.041 \\
(0.019)\end{array}$ & $\begin{array}{l}0.049 \\
(0.021)\end{array}$ & $\begin{array}{l}0.055 \\
(0.025)\end{array}$ & $\begin{array}{l}0.037 \\
(0.020)\end{array}$ & $\begin{array}{l}0.025 \\
(0.014)\end{array}$ \\
\hline Time Trend · Toscana & $\begin{array}{l}0.039 \\
(0.014)\end{array}$ & $\begin{array}{l}0.039 \\
(0.016)\end{array}$ & $\begin{array}{l}0.044 \\
(0.022)\end{array}$ & $\begin{array}{l}0.032 \\
(0.018)\end{array}$ & $\begin{array}{l}0.022 \\
(0.011)\end{array}$ \\
\hline Time Trend · Campania & $\begin{array}{l}0.026 \\
(0.014)\end{array}$ & $\begin{array}{l}0.022 \\
(0.013)\end{array}$ & $\begin{array}{l}0.034 \\
(0.012)\end{array}$ & $\begin{array}{l}0.028 \\
(0.011)\end{array}$ & $\begin{array}{l}0.019 \\
(0.010)\end{array}$ \\
\hline Time Trend $\cdot$ Sicilia & $\begin{array}{l}0.024 \\
(0.014)\end{array}$ & $\begin{array}{l}0.025 \\
(0.013)\end{array}$ & $\begin{array}{l}0.031 \\
(0.014)\end{array}$ & $\begin{array}{l}0.026 \\
(0.011)\end{array}$ & $\begin{array}{l}0.018 \\
(0.010)\end{array}$ \\
\hline \multicolumn{6}{|c|}{ Treatment province indicators } \\
\hline Time Trend $\cdot$ Monza & $\begin{array}{l}0.005 \\
(0.004)\end{array}$ & $\begin{array}{l}0.002 \\
(0.003)\end{array}$ & $\begin{array}{l}-0.011 \\
(0.011)\end{array}$ & $\begin{array}{l}0.007 \\
(0.008)\end{array}$ & $\begin{array}{l}-0.003 \\
(0.002)\end{array}$ \\
\hline Time Trend $\cdot$ Vicenza & $\begin{array}{l}0.009 \\
(0.009)\end{array}$ & $\begin{array}{l}0.002 \\
(0.005)\end{array}$ & $\begin{array}{l}0.003 \\
(0.005)\end{array}$ & $\begin{array}{l}-0.002 \\
(0.002)\end{array}$ & $\begin{array}{l}0.005 \\
(0.006)\end{array}$ \\
\hline Time Trend $\cdot$ Pisa & $\begin{array}{l}-0.009 \\
(0.010)\end{array}$ & $\begin{array}{l}-0.011 \\
(0.012)\end{array}$ & $\begin{array}{l}0.009 \\
(0.011)\end{array}$ & $\begin{array}{l}0.005 \\
(0.004)\end{array}$ & $\begin{array}{l}-0.006 \\
(0.005)\end{array}$ \\
\hline Time Trend $\cdot$ Salerno & $\begin{array}{l}-0.002 \\
(0.003)\end{array}$ & $\begin{array}{l}0.009 \\
(0.011)\end{array}$ & $\begin{array}{l}-0.008 \\
(0.009)\end{array}$ & $\begin{array}{l}0.006 \\
(0.007)\end{array}$ & $\begin{array}{l}-0.004 \\
(0.005)\end{array}$ \\
\hline Time Trend $\cdot$ Palermo & $\begin{array}{l}-0.009 \\
(0.011)\end{array}$ & $\begin{array}{l}0.006 \\
(0.009)\end{array}$ & $\begin{array}{l}-0.002 \\
(0.005) \\
\end{array}$ & $\begin{array}{l}-0.007 \\
(0.008)\end{array}$ & $\begin{array}{l}0.003 \\
(0.003)\end{array}$ \\
\hline Observations & 6,238 & 6,238 & 6,238 & 6,238 & 6,238 \\
\hline$F$-statistic & 0.89 & 0.77 & 0.51 & 0.38 & 0.45 \\
\hline
\end{tabular}

Notes. OLS regressions predicting outcomes in the pre-Productivity Program period for 804 firms that applied for management transfer (Panel A), 1,178 firms that applied for technology transfer (Panel B), and 1,612 firms that applied for the combined management and technology transfers (Panel C). Thirty firms whose applications were rejected are excluded. Data are provided at the firm level. Standard errors are block-bootstrapped at the province level with 200 replications. Lombardia, Veneto, Toscana, Campania, and Sicilia are indicator variables for pilot regions. Monza, Vicenza, Pisa, Salerno, and Palermo are indicator variables for treatment provinces. All the dependent variables are expressed in logs. Employment is the number of employees per firm; Assets, Sales, and Value Added are in 2010 USD, reevaluated from 1951 to 2010 values at 1 lira $=30.884$ euros and exchanged at 0.780 euro $=$ USD 1; TFPR is the logarithm of total factor productivity revenue, estimated using the Ackerberg, Caves and Frazer (2006) method. The $F$-statistics at the bottom of each panel test whether all the interaction terms between treatment provinces and the time trend are jointly zero. 
Table A.7-: Pre-Productivity Program Differences in Yearly Trends between Treated and Comparison Provinces, 1946-1951

\begin{tabular}{|c|c|c|c|c|c|c|c|c|c|c|}
\hline & \multicolumn{2}{|c|}{ Log Employment } & \multicolumn{2}{|c|}{ Log Assets } & \multicolumn{2}{|c|}{ Log Sales } & \multicolumn{2}{|c|}{ Log Value Added } & \multicolumn{2}{|c|}{ Log TFPR } \\
\hline & $(1)$ & $(2)$ & (3) & $(4)$ & $(5)$ & $(6)$ & $(7)$ & $(8)$ & $(9)$ & $(10)$ \\
\hline \multicolumn{11}{|l|}{ Panel A. Management } \\
\hline \multirow[t]{2}{*}{ Treatment Province 1947} & 0.012 & 0.007 & -0.010 & -0.014 & 0.021 & 0.022 & -0.009 & -0.005 & 0.006 & 0.004 \\
\hline & $(0.021)$ & $(0.019)$ & $(0.023)$ & $(0.026)$ & $(0.033)$ & $(0.021)$ & $(0.014)$ & $(0.013)$ & $(0.006)$ & $(0.007)$ \\
\hline \multirow[t]{2}{*}{ Treatment Province. 1948} & 0.006 & 0.009 & -0.014 & -0.009 & 0.017 & 0.014 & 0.004 & 0.003 & 0.012 & 0.010 \\
\hline & $(0.015)$ & $(0.025)$ & $(0.017)$ & $(0.012)$ & $(0.028)$ & $(0.009)$ & $(0.009)$ & $(0.009)$ & $(0.019)$ & $(0.014)$ \\
\hline \multirow[t]{2}{*}{ Treatment Province. 1949} & -0.009 & -0.015 & -0.009 & -0.003 & -0.010 & -0.009 & -0.012 & -0.011 & -0.007 & -0.008 \\
\hline & $(0.010)$ & $(0.029)$ & $(0.024)$ & $(0.009)$ & $(0.007)$ & $(0.012)$ & $(0.008)$ & $(0.014)$ & $(0.008)$ & $(0.016)$ \\
\hline \multirow[t]{2}{*}{ Treatment Province. 1950} & 0.008 & -0.007 & 0.007 & 0.011 & 0.008 & 0.009 & -0.012 & -0.014 & -0.004 & -0.005 \\
\hline & $(0.014)$ & $(0.009)$ & $(0.009)$ & $(0.021)$ & $(0.013)$ & $(0.012)$ & $(0.018)$ & $(0.029)$ & $(0.008)$ & $(0.008)$ \\
\hline \multirow[t]{2}{*}{ Treatment Province 1951} & 0.011 & 0.008 & -0.005 & -0.004 & -0.005 & -0.007 & 0.011 & 0.007 & -0.012 & -0.011 \\
\hline & $(0.024)$ & $(0.013)$ & $(0.012)$ & $(0.008)$ & $(0.017)$ & $(0.013)$ & $(0.015)$ & $(0.009)$ & $(0.014)$ & $(0.013)$ \\
\hline Observations & 3,141 & 3,141 & 3,141 & 3,141 & 3,141 & 3,141 & 3,141 & 3,141 & 3,141 & 3,141 \\
\hline$F$-statistic & 0.58 & 0.72 & 0.49 & 0.50 & 0.33 & 0.44 & 0.67 & 0.41 & 0.39 & 0.57 \\
\hline \multicolumn{11}{|l|}{ Panel B. Technology } \\
\hline \multirow[t]{2}{*}{ Treatment Province. 1947} & 0.013 & 0.009 & 0.015 & 0.006 & -0.004 & -0.004 & 0.007 & 0.006 & 0.013 & 0.014 \\
\hline & $(0.021)$ & $(0.014)$ & $(0.023)$ & $(0.006)$ & $(0.007)$ & $(0.006)$ & $(0.009)$ & $(0.009)$ & $(0.019)$ & $(0.018)$ \\
\hline \multirow[t]{2}{*}{ Treatment Province. 1948} & -0.002 & -0.004 & 0.013 & 0.012 & -0.010 & -0.014 & -0.015 & -0.019 & 0.011 & 0.009 \\
\hline & $(0.009)$ & $(0.009)$ & $(0.009)$ & $(0.019)$ & $(0.014)$ & $(0.017)$ & $(0.021)$ & $(0.023)$ & $(0.026)$ & $(0.019)$ \\
\hline \multirow[t]{2}{*}{ Treatment Province. 1949} & -0.009 & -0.012 & -0.011 & -0.012 & 0.008 & 0.009 & 0.009 & 0.014 & 0.014 & 0.017 \\
\hline & $(0.012)$ & $(0.008)$ & $(0.014)$ & $(0.008)$ & $(0.016)$ & $(0.015)$ & $(0.018)$ & $(0.024)$ & $(0.018)$ & $(0.023)$ \\
\hline \multirow[t]{2}{*}{ Treatment Province. 1950} & 0.018 & 0.012 & 0.014 & 0.014 & 0.005 & 0.008 & 0.024 & 0.021 & 0.016 & 0.019 \\
\hline & $(0.022)$ & $(0.018)$ & $(0.029)$ & $(0.028)$ & $(0.008)$ & $(0.014)$ & $(0.028)$ & $(0.029)$ & $(0.019)$ & $(0.021)$ \\
\hline \multirow[t]{2}{*}{ Treatment Province. 1951} & 0.007 & 0.011 & 0.007 & 0.012 & 0.011 & 0.012 & 0.015 & 0.013 & 0.003 & 0.005 \\
\hline & $(0.013)$ & $(0.015)$ & $(0.009)$ & $(0.014)$ & $(0.013)$ & $(0.015)$ & $(0.023)$ & $(0.026)$ & $(0.008)$ & $(0.009)$ \\
\hline Observations & 4,678 & 4,678 & 4,678 & 4,678 & 4,678 & 4,678 & 4,678 & 4,678 & 4,678 & 4,678 \\
\hline$F$-statistic & 0.21 & 0.59 & 0.83 & 0.26 & 0.69 & 0.41 & 0.58 & 0.44 & 0.39 & 0.42 \\
\hline Pilot region & Yes & Yes & Yes & Yes & Yes & Yes & Yes & Yes & Yes & Yes \\
\hline Pilot region $\mathrm{x}$ time $\mathrm{FE}$ & No & Yes & No & Yes & No & Yes & No & Yes & No & Yes \\
\hline
\end{tabular}


Table A.7-: Continued

\begin{tabular}{|c|c|c|c|c|c|c|c|c|c|c|}
\hline & \multicolumn{2}{|c|}{ Log Employment } & \multicolumn{2}{|c|}{ Log Assets } & \multicolumn{2}{|c|}{ Log Sales } & \multicolumn{2}{|c|}{ Log Value Added } & \multicolumn{2}{|c|}{ Log TFPR } \\
\hline & $(1)$ & $(2)$ & $(3)$ & $(4)$ & $(5)$ & $(6)$ & $(7)$ & $(8)$ & $(9)$ & $(10)$ \\
\hline \multicolumn{11}{|l|}{ Panel C. Combined } \\
\hline \multirow{2}{*}{ Treatment Province 1947} & -0.018 & -0.015 & 0.023 & 0.024 & 0.014 & 0.012 & 0.017 & 0.015 & 0.005 & 0.004 \\
\hline & $(0.021)$ & $(0.018)$ & $(0.026)$ & $(0.025)$ & $(0.012)$ & $(0.011)$ & $(0.019)$ & $(0.018)$ & $(0.006)$ & $(0.006)$ \\
\hline \multirow[t]{2}{*}{ Treatment Province 1948} & 0.009 & 0.009 & 0.015 & 0.013 & -0.007 & 0.006 & -0.011 & -0.009 & 0.002 & 0.002 \\
\hline & $(0.012)$ & $(0.011)$ & $(0.019)$ & $(0.017)$ & $(0.008)$ & $(0.009)$ & $(0.012)$ & $(0.010)$ & $(0.003)$ & $(0.004)$ \\
\hline \multirow[t]{2}{*}{ Treatment Province 1949} & 0.007 & 0.008 & -0.003 & -0.002 & 0.005 & 0.006 & 0.015 & 0.017 & 0.004 & 0.003 \\
\hline & $(0.009)$ & $(0.009)$ & $(0.006)$ & $(0.004)$ & $(0.004)$ & $(0.007)$ & $(0.016)$ & $(0.014)$ & $(0.004)$ & $(0.004)$ \\
\hline \multirow[t]{2}{*}{ Treatment Province 1950} & 0.010 & 0.012 & 0.009 & 0.006 & -0.011 & -0.008 & -0.011 & -0.011 & 0.004 & 0.005 \\
\hline & $(0.015)$ & $(0.014)$ & $(0.011)$ & $(0.009)$ & $(0.013)$ & $(0.012)$ & $(0.012)$ & $(0.011)$ & $(0.005)$ & $(0.005)$ \\
\hline \multirow[t]{2}{*}{ Treatment Province 1951} & -0.017 & -0.016 & 0.011 & 0.014 & 0.003 & 0.003 & 0.004 & 0.006 & -0.002 & -0.002 \\
\hline & $(0.021)$ & $(0.020)$ & $(0.015)$ & $(0.013)$ & $(0.007)$ & $(0.008)$ & $(0.005)$ & $(0.005)$ & $(0.004)$ & $(0.003)$ \\
\hline Observations & 6,238 & 6,238 & 6,238 & 6,238 & 6,238 & 6,238 & 6,238 & 6,238 & 6,238 & 6,238 \\
\hline$F$-statistic & 0.59 & 0.68 & 0.91 & 0.65 & 0.63 & 0.65 & 0.49 & 0.81 & 0.39 & 0.42 \\
\hline Pilot region & Yes & Yes & Yes & Yes & Yes & Yes & Yes & Yes & Yes & Yes \\
\hline Pilot region $\mathrm{x}$ time $\mathrm{FE}$ & No & Yes & No & Yes & No & Yes & No & Yes & No & Yes \\
\hline
\end{tabular}

Notes. OLS regressions predicting outcomes in the pre-Productivity Program period for 804 firms that applied for management transfer (Panel A), 1,178 firms that applied for technology transfer (Panel B), and 1,612 firms that applied for the combined management and technology transfers (Panel C). Thirty firms whose applications were rejected are excluded. Data are provided at the firm level. The trend is allowed to vary freely for each year before the Productivity Program was implemented. Year dummies are included, but their coefficients are not reported. Standard errors are block-bootstrapped at the province level with 200 replications. All the dependent variables are expressed in logs. Employment is the number of employees per firm; Assets, Sales, and Value Added are in 2010 USD, reevaluated from 1951 to 2010 values at 1 lira $=30.884$ euros and exchanged at 0.780 euro=USD $1 ;$ TFPR is the logarithm of total factor productivity revenue, estimated using the Ackerberg, Caves and Frazer (2006) method. The $F$-statistics at the bottom of each panel test whether all the interaction terms between treatment provinces and the year dummy variables are jointly zero. 
Table A.8-: Pre-Productivity Program Differences in Yearly Province Time Trends, 1946-1951

\begin{tabular}{|c|c|c|c|c|c|}
\hline & $\begin{array}{l}\text { Log Empl. } \\
\text { (1) }\end{array}$ & $\begin{array}{l}\text { Log Assets } \\
(2)\end{array}$ & $\begin{array}{l}\text { Log Sales } \\
(3)\end{array}$ & $\begin{array}{l}\text { Log Value Added } \\
(4)\end{array}$ & $\begin{array}{l}\text { Log TFPR } \\
\text { (5) }\end{array}$ \\
\hline \multicolumn{6}{|c|}{ Panel A. Management } \\
\hline Monza $\cdot 1947$ & $\begin{array}{l}0.013 \\
(0.015)\end{array}$ & $\begin{array}{l}0.006 \\
(0.008)\end{array}$ & $\begin{array}{l}0.010 \\
(0.009)\end{array}$ & $\begin{array}{l}0.003 \\
(0.004)\end{array}$ & $\begin{array}{l}-0.007 \\
(0.009)\end{array}$ \\
\hline Monza $\cdot 1948$ & $\begin{array}{l}0.007 \\
(0.006)\end{array}$ & $\begin{array}{l}0.010 \\
(0.008)\end{array}$ & $\begin{array}{l}-0.009 \\
(0.011)\end{array}$ & $\begin{array}{l}-0.006 \\
(0.007)\end{array}$ & $\begin{array}{l}0.004 \\
(0.006)\end{array}$ \\
\hline Monza $\cdot 1949$ & $\begin{array}{l}-0.011 \\
(0.012)\end{array}$ & $\begin{array}{l}0.011 \\
(0.015)\end{array}$ & $\begin{array}{l}0.005 \\
(0.006)\end{array}$ & $\begin{array}{l}0.013 \\
(0.011)\end{array}$ & $\begin{array}{l}-0.011 \\
(0.013)\end{array}$ \\
\hline Monza $\cdot 1950$ & $\begin{array}{l}0.013 \\
(0.010)\end{array}$ & $\begin{array}{l}0.009 \\
(0.011)\end{array}$ & $\begin{array}{l}-0.011 \\
(0.012)\end{array}$ & $\begin{array}{l}-0.007 \\
(0.009)\end{array}$ & $\begin{array}{l}0.008 \\
(0.008)\end{array}$ \\
\hline Monza $\cdot 1951$ & $\begin{array}{l}-0.023 \\
(0.028)\end{array}$ & $\begin{array}{l}0.012 \\
(0.011)\end{array}$ & $\begin{array}{l}0.005 \\
(0.007)\end{array}$ & $\begin{array}{l}0.009 \\
(0.010)\end{array}$ & $\begin{array}{l}0.006 \\
(0.007)\end{array}$ \\
\hline Vicenza $\cdot 1947$ & $\begin{array}{l}0.008 \\
(0.009)\end{array}$ & $\begin{array}{l}-0.007 \\
(0.006)\end{array}$ & $\begin{array}{l}0.015 \\
(0.011)\end{array}$ & $\begin{array}{l}0.004 \\
(0.005)\end{array}$ & $\begin{array}{l}-0.007 \\
(0.005)\end{array}$ \\
\hline Vicenza $\cdot 1948$ & $\begin{array}{l}0.010 \\
(0.008)\end{array}$ & $\begin{array}{l}-0.004 \\
(0.005)\end{array}$ & $\begin{array}{l}-0.007 \\
(0.009)\end{array}$ & $\begin{array}{l}-0.014 \\
(0.009)\end{array}$ & $\begin{array}{l}0.004 \\
(0.009)\end{array}$ \\
\hline Vicenza $\cdot 1949$ & $\begin{array}{l}-0.017 \\
(0.024)\end{array}$ & $\begin{array}{l}0.012 \\
(0.014)\end{array}$ & $\begin{array}{l}0.003 \\
(0.005)\end{array}$ & $\begin{array}{l}0.008 \\
(0.009)\end{array}$ & $\begin{array}{l}-0.005 \\
(0.006)\end{array}$ \\
\hline Vicenza $\cdot 1950$ & $\begin{array}{l}0.011 \\
(0.012)\end{array}$ & $\begin{array}{l}-0.009 \\
(0.009)\end{array}$ & $\begin{array}{l}-0.012 \\
(0.015)\end{array}$ & $\begin{array}{l}-0.009 \\
(0.010)\end{array}$ & $\begin{array}{l}0.009 \\
(0.008)\end{array}$ \\
\hline Vicenza $\cdot 1951$ & $\begin{array}{l}0.009 \\
(0.008)\end{array}$ & $\begin{array}{l}0.006 \\
(0.007)\end{array}$ & $\begin{array}{l}0.007 \\
(0.006)\end{array}$ & $\begin{array}{l}0.006 \\
(0.006)\end{array}$ & $\begin{array}{l}-0.011 \\
(0.012)\end{array}$ \\
\hline Pisa $\cdot 1947$ & $\begin{array}{l}0.013 \\
(0.012)\end{array}$ & $\begin{array}{l}0.012 \\
(0.015)\end{array}$ & $\begin{array}{l}-0.006 \\
(0.005)\end{array}$ & $\begin{array}{l}0.008 \\
(0.010)\end{array}$ & $\begin{array}{l}0.008 \\
(0.009)\end{array}$ \\
\hline Pisa $\cdot 1948$ & $\begin{array}{l}-0.015 \\
(0.013)\end{array}$ & $\begin{array}{l}-0.005 \\
(0.007)\end{array}$ & $\begin{array}{l}0.012 \\
(0.009)\end{array}$ & $\begin{array}{l}-0.004 \\
(0.005)\end{array}$ & $\begin{array}{l}0.007 \\
(0.006)\end{array}$ \\
\hline Pisa $\cdot 1949$ & $\begin{array}{l}0.017 \\
(0.015)\end{array}$ & $\begin{array}{l}0.011 \\
(0.012)\end{array}$ & $\begin{array}{l}0.009 \\
(0.010)\end{array}$ & $\begin{array}{l}0.012 \\
(0.008)\end{array}$ & $\begin{array}{l}-0.004 \\
(0.007)\end{array}$ \\
\hline Pisa $\cdot 1950$ & $\begin{array}{l}0.007 \\
(0.009)\end{array}$ & $\begin{array}{l}0.006 \\
(0.007)\end{array}$ & $\begin{array}{l}-0.005 \\
(0.004)\end{array}$ & $\begin{array}{l}-0.009 \\
(0.012)\end{array}$ & $\begin{array}{l}-0.005 \\
(0.005)\end{array}$ \\
\hline Pisa $\cdot 1951$ & $\begin{array}{l}-0.004 \\
(0.005)\end{array}$ & $\begin{array}{l}-0.010 \\
(0.012)\end{array}$ & $\begin{array}{l}0.013 \\
(0.015)\end{array}$ & $\begin{array}{l}0.005 \\
(0.006)\end{array}$ & $\begin{array}{l}0.003 \\
(0.004)\end{array}$ \\
\hline Salerno $\cdot 1947$ & $\begin{array}{l}0.014 \\
(0.011)\end{array}$ & $\begin{array}{l}-0.005 \\
(0.004)\end{array}$ & $\begin{array}{l}-0.012 \\
(0.015)\end{array}$ & $\begin{array}{l}0.006 \\
(0.009)\end{array}$ & $\begin{array}{l}0.012 \\
(0.008)\end{array}$ \\
\hline Salerno $\cdot 1948$ & $\begin{array}{l}-0.016 \\
(0.013)\end{array}$ & $\begin{array}{l}0.007 \\
(0.008)\end{array}$ & $\begin{array}{l}0.004 \\
(0.006)\end{array}$ & $\begin{array}{l}-0.010 \\
(0.011)\end{array}$ & $\begin{array}{l}-0.008 \\
(0.009)\end{array}$ \\
\hline Salerno $\cdot 1949$ & $\begin{array}{l}0.008 \\
(0.006)\end{array}$ & $\begin{array}{l}0.006 \\
(0.007)\end{array}$ & $\begin{array}{l}-0.009 \\
(0.013)\end{array}$ & $\begin{array}{l}-0.014 \\
(0.016)\end{array}$ & $\begin{array}{l}0.011 \\
(0.014)\end{array}$ \\
\hline Salerno $\cdot 1950$ & $\begin{array}{l}-0.010 \\
(0.008)\end{array}$ & $\begin{array}{l}-0.009 \\
(0.010)\end{array}$ & $\begin{array}{l}0.011 \\
(0.014)\end{array}$ & $\begin{array}{l}0.008 \\
(0.009)\end{array}$ & $\begin{array}{l}0.003 \\
(0.005)\end{array}$ \\
\hline Salerno $\cdot 1951$ & $\begin{array}{l}0.009 \\
(0.011)\end{array}$ & $\begin{array}{l}0.012 \\
(0.008)\end{array}$ & $\begin{array}{l}0.005 \\
(0.006)\end{array}$ & $\begin{array}{l}0.005 \\
(0.006)\end{array}$ & $\begin{array}{l}-0.005 \\
(0.007)\end{array}$ \\
\hline
\end{tabular}


Table A.8-: Continued

\begin{tabular}{|c|c|c|c|c|c|}
\hline & $\begin{array}{l}\text { Log Empl. } \\
\text { (1) }\end{array}$ & $\begin{array}{l}\text { Log Assets } \\
(2)\end{array}$ & $\begin{array}{l}\text { Log Sales } \\
(3)\end{array}$ & $\begin{array}{l}\text { Log Value Added } \\
\text { (4) }\end{array}$ & $\begin{array}{l}\text { Log TFPR } \\
\text { (5) }\end{array}$ \\
\hline Palermo $\cdot 1947$ & $\begin{array}{l}0.008 \\
(0.009)\end{array}$ & $\begin{array}{l}0.014 \\
(0.012)\end{array}$ & $\begin{array}{l}-0.004 \\
(0.008)\end{array}$ & $\begin{array}{l}0.011 \\
(0.013)\end{array}$ & $\begin{array}{l}-0.009 \\
(0.008)\end{array}$ \\
\hline Palermo. 1948 & $\begin{array}{l}0.014 \\
(0.010)\end{array}$ & $\begin{array}{l}-0.009 \\
(0.011)\end{array}$ & $\begin{array}{l}0.006 \\
(0.008)\end{array}$ & $\begin{array}{l}-0.012 \\
(0.016)\end{array}$ & $\begin{array}{l}0.004 \\
(0.005)\end{array}$ \\
\hline Palermo 1949 & $\begin{array}{l}-0.005 \\
(0.006)\end{array}$ & $\begin{array}{l}0.006 \\
(0.008)\end{array}$ & $\begin{array}{l}0.008 \\
(0.009)\end{array}$ & $\begin{array}{l}0.008 \\
(0.009)\end{array}$ & $\begin{array}{l}0.006 \\
(0.007)\end{array}$ \\
\hline Palermo 1950 & $\begin{array}{l}0.007 \\
(0.009)\end{array}$ & $\begin{array}{l}-0.005 \\
(0.007)\end{array}$ & $\begin{array}{l}-0.004) \\
(0.005)\end{array}$ & $\begin{array}{l}-0.005 \\
(0.007)\end{array}$ & $\begin{array}{l}-0.008 \\
(0.009)\end{array}$ \\
\hline Palermo. 1951 & $\begin{array}{l}-0.010 \\
(0.007)\end{array}$ & $\begin{array}{l}0.003 \\
(0.002)\end{array}$ & $\begin{array}{l}0.002 \\
(0.005)\end{array}$ & $\begin{array}{l}0.006 \\
(0.006)\end{array}$ & $\begin{array}{l}-0.004 \\
(0.005)\end{array}$ \\
\hline Observations & 3,141 & 3,141 & 3,141 & 3,141 & 3,141 \\
\hline$F$-statistic & 0.56 & 0.73 & 0.49 & 0.55 & 0.72 \\
\hline \multicolumn{6}{|c|}{ Panel B. Technology } \\
\hline Monza $\cdot 1947$ & $\begin{array}{l}-0.011 \\
(0.013)\end{array}$ & $\begin{array}{l}0.006 \\
(0.009)\end{array}$ & $\begin{array}{l}-0.008 \\
(0.006)\end{array}$ & $\begin{array}{l}0.004 \\
(0.005)\end{array}$ & $\begin{array}{l}-0.002 \\
(0.002)\end{array}$ \\
\hline Monza $\cdot 1948$ & $\begin{array}{l}0.005 \\
(0.006)\end{array}$ & $\begin{array}{l}-0.008 \\
(0.010)\end{array}$ & $\begin{array}{l}0.005 \\
(0.004)\end{array}$ & $\begin{array}{l}-0.008 \\
(0.009)\end{array}$ & $\begin{array}{l}-0.003 \\
(0.005)\end{array}$ \\
\hline Monza $\cdot 1949$ & $\begin{array}{l}-0.012 \\
(0.010)\end{array}$ & $\begin{array}{l}0.011 \\
(0.009)\end{array}$ & $\begin{array}{l}0.006 \\
(0.005)\end{array}$ & $\begin{array}{l}0.011 \\
(0.009)\end{array}$ & $\begin{array}{l}0.004 \\
(0.003)\end{array}$ \\
\hline Monza $\cdot 1950$ & $\begin{array}{l}-0.009 \\
(0.007)\end{array}$ & $\begin{array}{l}-0.012 \\
(0.008)\end{array}$ & $\begin{array}{l}-0.011 \\
(0.012)\end{array}$ & $\begin{array}{l}0.007 \\
(0.006)\end{array}$ & $\begin{array}{l}0.005 \\
(0.006)\end{array}$ \\
\hline Monza $\cdot 1951$ & $\begin{array}{l}0.004 \\
(0.006)\end{array}$ & $\begin{array}{l}-0.014 \\
(0.015)\end{array}$ & $\begin{array}{l}0.009 \\
(0.007)\end{array}$ & $\begin{array}{l}-0.013 \\
(0.011)\end{array}$ & $\begin{array}{l}-0.011 \\
(0.012)\end{array}$ \\
\hline Vicenza $\cdot 1947$ & $\begin{array}{l}0.007 \\
(0.009)\end{array}$ & $\begin{array}{l}0.004 \\
(0.007)\end{array}$ & $\begin{array}{l}-0.004 \\
(0.009)\end{array}$ & $\begin{array}{l}0.015 \\
(0.016)\end{array}$ & $\begin{array}{l}0.009 \\
(0.009)\end{array}$ \\
\hline Vicenza $\cdot 1948$ & $\begin{array}{l}-0.006 \\
(0.006)\end{array}$ & $\begin{array}{l}-0.003 \\
(0.005)\end{array}$ & $\begin{array}{l}0.004 \\
(0.003)\end{array}$ & $\begin{array}{l}-0.009 \\
(0.009)\end{array}$ & $\begin{array}{l}0.006 \\
(0.008)\end{array}$ \\
\hline Vicenza. 1949 & $\begin{array}{l}-0.011 \\
(0.015)\end{array}$ & $\begin{array}{l}0.002 \\
(0.003)\end{array}$ & $\begin{array}{l}0.005 \\
(0.007)\end{array}$ & $\begin{array}{l}0.007 \\
(0.008)\end{array}$ & $\begin{array}{l}-0.007 \\
(0.005)\end{array}$ \\
\hline Vicenza $\cdot 1950$ & $\begin{array}{l}0.008 \\
(0.008)\end{array}$ & $\begin{array}{l}0.009 \\
(0.008)\end{array}$ & $\begin{array}{l}-0.006 \\
(0.005)\end{array}$ & $\begin{array}{l}-0.008 \\
(0.009)\end{array}$ & $\begin{array}{l}0.011 \\
(0.019)\end{array}$ \\
\hline Vicenza $\cdot 1951$ & $\begin{array}{l}0.004 \\
(0.006)\end{array}$ & $\begin{array}{l}-0.006 \\
(0.008)\end{array}$ & $\begin{array}{l}0.011 \\
(0.013)\end{array}$ & $\begin{array}{l}0.014 \\
(0.011)\end{array}$ & $\begin{array}{l}0.012 \\
(0.013)\end{array}$ \\
\hline Pisa $\cdot 1947$ & $\begin{array}{l}-0.009 \\
(0.011)\end{array}$ & $\begin{array}{l}0.005 \\
(0.004)\end{array}$ & $\begin{array}{l}-0.015 \\
(0.011)\end{array}$ & $\begin{array}{l}0.006 \\
(0.004)\end{array}$ & $\begin{array}{l}-0.014 \\
(0.012)\end{array}$ \\
\hline Pisa $\cdot 1948$ & $\begin{array}{l}0.012 \\
(0.014)\end{array}$ & $\begin{array}{l}0.007 \\
(0.005)\end{array}$ & $\begin{array}{l}0.007 \\
(0.009)\end{array}$ & $\begin{array}{l}-0.008 \\
(0.007)\end{array}$ & $\begin{array}{l}0.005 \\
(0.004)\end{array}$ \\
\hline Pisa $\cdot 1949$ & $\begin{array}{l}0.013 \\
(0.012)\end{array}$ & $\begin{array}{l}-0.008 \\
(0.009)\end{array}$ & $\begin{array}{l}0.003 \\
(0.002)\end{array}$ & $\begin{array}{l}0.010 \\
(0.012)\end{array}$ & $\begin{array}{l}0.008 \\
(0.005)\end{array}$ \\
\hline Pisa $\cdot 1950$ & $\begin{array}{l}-0.011 \\
(0.009)\end{array}$ & $\begin{array}{l}0.004 \\
(0.006)\end{array}$ & $\begin{array}{l}-0.009 \\
(0.009)\end{array}$ & $\begin{array}{l}-0.013 \\
(0.019)\end{array}$ & $\begin{array}{l}-0.005 \\
(0.008)\end{array}$ \\
\hline Pisa $\cdot 1951$ & $\begin{array}{l}-0.005 \\
(0.004)\end{array}$ & $\begin{array}{l}-0.011 \\
(0.014)\end{array}$ & $\begin{array}{l}0.014 \\
(0.016)\end{array}$ & $\begin{array}{l}0.004 \\
(0.005)\end{array}$ & $\begin{array}{l}0.006 \\
(0.005)\end{array}$ \\
\hline
\end{tabular}


Table A.8-: Continued

\begin{tabular}{|c|c|c|c|c|c|}
\hline & $\begin{array}{l}\text { Log Empl. } \\
\text { (1) }\end{array}$ & $\begin{array}{l}\text { Log Assets } \\
(2)\end{array}$ & $\begin{array}{l}\text { Log Sales } \\
(3)\end{array}$ & $\begin{array}{l}\text { Log Value Added } \\
\text { (4) }\end{array}$ & $\begin{array}{l}\text { Log TFPR } \\
\text { (5) }\end{array}$ \\
\hline Salerno $\cdot 1947$ & $\begin{array}{l}-0.004 \\
(0.007)\end{array}$ & $\begin{array}{l}0.005 \\
(0.008)\end{array}$ & $\begin{array}{l}0.006 \\
(0.008)\end{array}$ & $\begin{array}{l}-0.009 \\
(0.009)\end{array}$ & $\begin{array}{l}0.011 \\
(0.008)\end{array}$ \\
\hline Salerno $\cdot 1948$ & $\begin{array}{l}0.006 \\
(0.008)\end{array}$ & $\begin{array}{l}-0.007 \\
(0.009)\end{array}$ & $\begin{array}{l}-0.004 \\
(0.005)\end{array}$ & $\begin{array}{l}0.004 \\
(0.006)\end{array}$ & $\begin{array}{l}0.009 \\
(0.008)\end{array}$ \\
\hline Salerno $\cdot 1949$ & $\begin{array}{l}0.005 \\
(0.005)\end{array}$ & $\begin{array}{l}0.003 \\
(0.002)\end{array}$ & $\begin{array}{l}-0.009 \\
(0.007)\end{array}$ & $\begin{array}{l}0.011 \\
(0.014)\end{array}$ & $\begin{array}{l}0.004 \\
(0.005)\end{array}$ \\
\hline Salerno $\cdot 1950$ & $\begin{array}{l}0.007 \\
(0.005)\end{array}$ & $\begin{array}{l}0.014 \\
(0.016)\end{array}$ & $\begin{array}{l}0.011 \\
(0.010)\end{array}$ & $\begin{array}{l}0.012 \\
(0.014)\end{array}$ & $\begin{array}{l}-0.007 \\
(0.008)\end{array}$ \\
\hline Salerno $\cdot 1951$ & $\begin{array}{l}-0.004 \\
(0.007)\end{array}$ & $\begin{array}{l}-0.002 \\
(0.006)\end{array}$ & $\begin{array}{l}0.013 \\
(0.016)\end{array}$ & $\begin{array}{l}-0.007 \\
(0.009)\end{array}$ & $\begin{array}{l}0.011 \\
(0.014)\end{array}$ \\
\hline Palermo $\cdot 1947$ & $\begin{array}{l}0.011 \\
(0.012)\end{array}$ & $\begin{array}{l}0.008 \\
(0.010)\end{array}$ & $\begin{array}{l}-0.011 \\
(0.009)\end{array}$ & $\begin{array}{l}0.006 \\
(0.008)\end{array}$ & $\begin{array}{l}-0.015 \\
(0.011)\end{array}$ \\
\hline Palermo. 1948 & $\begin{array}{l}0.002 \\
(0.004)\end{array}$ & $\begin{array}{l}0.006 \\
(0.005)\end{array}$ & $\begin{array}{l}0.007 \\
(0.007)\end{array}$ & $\begin{array}{l}-0.005 \\
(0.004)\end{array}$ & $\begin{array}{l}0.008 \\
(0.006)\end{array}$ \\
\hline Palermo. 1949 & $\begin{array}{l}-0.005 \\
(0.006)\end{array}$ & $\begin{array}{l}-0.013 \\
(0.009)\end{array}$ & $\begin{array}{l}-0.007 \\
(0.009)\end{array}$ & $\begin{array}{l}0.008 \\
(0.006)\end{array}$ & $\begin{array}{l}0.009 \\
(0.011)\end{array}$ \\
\hline Palermo 1950 & $\begin{array}{l}0.014 \\
(0.011)\end{array}$ & $\begin{array}{l}0.005 \\
(0.007)\end{array}$ & $\begin{array}{l}0.004 \\
(0.008)\end{array}$ & $\begin{array}{l}-0.009 \\
(0.007)\end{array}$ & $\begin{array}{l}-0.002 \\
(0.002)\end{array}$ \\
\hline Palermo 1951 & $\begin{array}{l}-0.008 \\
(0.005)\end{array}$ & $\begin{array}{l}0.009 \\
(0.011)\end{array}$ & $\begin{array}{l}-0.005 \\
(0.006)\end{array}$ & $\begin{array}{l}0.003 \\
(0.004)\end{array}$ & $\begin{array}{l}0.003 \\
(0.004)\end{array}$ \\
\hline Observations & 4,678 & 4,678 & 4,678 & 4,678 & 4,678 \\
\hline$F$-statistic & 0.72 & 0.94 & 0.54 & 0.91 & 0.67 \\
\hline \multicolumn{6}{|c|}{ Panel C. Combined } \\
\hline Monza $\cdot 1947$ & $\begin{array}{l}-0.005 \\
(0.007)\end{array}$ & $\begin{array}{l}0.003 \\
(0.005)\end{array}$ & $\begin{array}{l}0.007 \\
(0.008)\end{array}$ & $\begin{array}{l}-0.009 \\
(0.007)\end{array}$ & $\begin{array}{l}-0.007 \\
(0.009)\end{array}$ \\
\hline Monza $\cdot 1948$ & $\begin{array}{l}0.008 \\
(0.009)\end{array}$ & $\begin{array}{l}0.007 \\
(0.006)\end{array}$ & $\begin{array}{l}-0.009 \\
(0.011)\end{array}$ & $\begin{array}{l}0.013 \\
(0.014)\end{array}$ & $\begin{array}{l}0.006 \\
(0.007)\end{array}$ \\
\hline Monza $\cdot 1949$ & $\begin{array}{l}-0.006 \\
(0.005)\end{array}$ & $\begin{array}{l}-0.009 \\
(0.009)\end{array}$ & $\begin{array}{l}0.004 \\
(0.006)\end{array}$ & $\begin{array}{l}-0.004 \\
(0.007)\end{array}$ & $\begin{array}{l}-0.005 \\
(0.005)\end{array}$ \\
\hline Monza $\cdot 1950$ & $\begin{array}{l}0.007 \\
(0.008)\end{array}$ & $\begin{array}{l}0.013 \\
(0.014)\end{array}$ & $\begin{array}{l}-0.003 \\
(0.005)\end{array}$ & $\begin{array}{l}0.005 \\
(0.006)\end{array}$ & $\begin{array}{l}0.004 \\
(0.007)\end{array}$ \\
\hline Monza $\cdot 1951$ & $\begin{array}{l}-0.004 \\
(0.006)\end{array}$ & $\begin{array}{l}0.005 \\
(0.007)\end{array}$ & $\begin{array}{l}0.013 \\
(0.012)\end{array}$ & $\begin{array}{l}0.002 \\
(0.002)\end{array}$ & $\begin{array}{l}0.010 \\
(0.011)\end{array}$ \\
\hline Vicenza $\cdot 1947$ & $\begin{array}{l}-0.003 \\
(0.004)\end{array}$ & $\begin{array}{l}-0.004 \\
(0.003)\end{array}$ & $\begin{array}{l}0.007 \\
(0.009)\end{array}$ & $\begin{array}{l}-0.004 \\
(0.005)\end{array}$ & $\begin{array}{l}-0.002 \\
(0.004)\end{array}$ \\
\hline Vicenza $\cdot 1948$ & $\begin{array}{l}0.002 \\
(0.003)\end{array}$ & $\begin{array}{l}0.005 \\
(0.005)\end{array}$ & $\begin{array}{l}-0.003 \\
(0.005)\end{array}$ & $\begin{array}{l}0.006 \\
(0.007)\end{array}$ & $\begin{array}{l}0.006 \\
(0.007)\end{array}$ \\
\hline Vicenza $\cdot 1949$ & $\begin{array}{l}0.011 \\
(0.015)\end{array}$ & $\begin{array}{l}0.008 \\
(0.011)\end{array}$ & $\begin{array}{l}0.009 \\
(0.010)\end{array}$ & $\begin{array}{l}-0.011 \\
(0.012)\end{array}$ & $\begin{array}{l}-0.004 \\
(0.008)\end{array}$ \\
\hline Vicenza $\cdot 1950$ & $\begin{array}{l}-0.009 \\
(0.008)\end{array}$ & $\begin{array}{l}-0.014 \\
(0.011)\end{array}$ & $\begin{array}{l}-0.012 \\
(0.011)\end{array}$ & $\begin{array}{l}0.002 \\
(0.006)\end{array}$ & $\begin{array}{l}0.012 \\
(0.020)\end{array}$ \\
\hline Vicenza $\cdot 1951$ & $\begin{array}{l}0.004 \\
(0.004)\end{array}$ & $\begin{array}{l}0.007 \\
(0.008)\end{array}$ & $\begin{array}{l}0.007 \\
(0.006)\end{array}$ & $\begin{array}{l}0.004 \\
(0.005)\end{array}$ & $\begin{array}{l}-0.007 \\
(0.009)\end{array}$ \\
\hline
\end{tabular}


Table A.8-: Continued

\begin{tabular}{|c|c|c|c|c|c|}
\hline & $\begin{array}{l}\text { Log Empl. } \\
\text { (1) }\end{array}$ & $\begin{array}{l}\text { Log Assets } \\
(2)\end{array}$ & $\begin{array}{l}\text { Log Sales } \\
(3)\end{array}$ & $\begin{array}{l}\text { Log Value Added } \\
(4)\end{array}$ & $\begin{array}{l}\text { Log TFPR } \\
\text { (5) }\end{array}$ \\
\hline Pisa $\cdot 1947$ & $\begin{array}{l}-0.005 \\
(0.006)\end{array}$ & $\begin{array}{l}0.003 \\
(0.005)\end{array}$ & $\begin{array}{l}-0.008 \\
(0.007)\end{array}$ & $\begin{array}{l}-0.008 \\
(0.009)\end{array}$ & $\begin{array}{l}-0.008 \\
(0.005)\end{array}$ \\
\hline Pisa $\cdot 1948$ & $\begin{array}{l}0.006 \\
(0.004)\end{array}$ & $\begin{array}{l}-0.006 \\
(0.007)\end{array}$ & $\begin{array}{l}0.001 \\
(0.001)\end{array}$ & $\begin{array}{l}0.006 \\
(0.004)\end{array}$ & $\begin{array}{l}-0.003 \\
(0.004)\end{array}$ \\
\hline Pisa $\cdot 1949$ & $\begin{array}{l}0.002 \\
(0.003)\end{array}$ & $\begin{array}{l}0.008 \\
(0.006)\end{array}$ & $\begin{array}{l}-0.005 \\
(0.006)\end{array}$ & $\begin{array}{l}-0.001 \\
(0.002)\end{array}$ & $\begin{array}{l}0.002 \\
(0.005)\end{array}$ \\
\hline Pisa $\cdot 1950$ & $\begin{array}{l}0.005 \\
(0.004)\end{array}$ & $\begin{array}{l}-0.003 \\
(0.005)\end{array}$ & $\begin{array}{l}0.009 \\
(0.010)\end{array}$ & $\begin{array}{l}0.012 \\
(0.008)\end{array}$ & $\begin{array}{l}0.006 \\
(0.004)\end{array}$ \\
\hline Pisa $\cdot 1951$ & $\begin{array}{l}0.012 \\
(0.013)\end{array}$ & $\begin{array}{l}0.004 \\
(0.006)\end{array}$ & $\begin{array}{l}-0.012 \\
(0.015)\end{array}$ & $\begin{array}{l}0.007 \\
(0.009)\end{array}$ & $\begin{array}{l}0.008 \\
(0.008)\end{array}$ \\
\hline Salerno $\cdot 1947$ & $\begin{array}{l}0.008 \\
(0.008)\end{array}$ & $\begin{array}{l}0.008 \\
(0.009)\end{array}$ & $\begin{array}{l}0.004 \\
(0.008)\end{array}$ & $\begin{array}{l}0.005 \\
(0.006)\end{array}$ & $\begin{array}{l}-0.001 \\
(0.002)\end{array}$ \\
\hline Salerno $\cdot 1948$ & $\begin{array}{l}-0.003 \\
(0.006)\end{array}$ & $\begin{array}{l}-0.011 \\
(0.013)\end{array}$ & $\begin{array}{l}-0.008 \\
(0.009)\end{array}$ & $\begin{array}{l}-0.004 \\
(0.006)\end{array}$ & $\begin{array}{l}0.003 \\
(0.004)\end{array}$ \\
\hline Salerno $\cdot 1949$ & $\begin{array}{l}0.005 \\
(0.004)\end{array}$ & $\begin{array}{l}-0.005 \\
(0.007)\end{array}$ & $\begin{array}{l}0.013 \\
(0.016)\end{array}$ & $\begin{array}{l}0.005 \\
(0.006)\end{array}$ & $\begin{array}{l}-0.005 \\
(0.008)\end{array}$ \\
\hline Salerno $\cdot 1950$ & $\begin{array}{l}0.006 \\
(0.007)\end{array}$ & $\begin{array}{l}0.006 \\
(0.005)\end{array}$ & $\begin{array}{l}-0.011 \\
(0.014)\end{array}$ & $\begin{array}{l}0.006 \\
(0.009)\end{array}$ & $\begin{array}{l}0.012 \\
(0.011)\end{array}$ \\
\hline Salerno $\cdot 1951$ & $\begin{array}{l}-0.009 \\
(0.009)\end{array}$ & $\begin{array}{l}0.009 \\
(0.012)\end{array}$ & $\begin{array}{l}0.007 \\
(0.006)\end{array}$ & $\begin{array}{l}-0.003 \\
(0.004)\end{array}$ & $\begin{array}{l}0.006 \\
(0.008)\end{array}$ \\
\hline Palermo $\cdot 1947$ & $\begin{array}{l}0.011 \\
(0.013)\end{array}$ & $\begin{array}{l}-0.007 \\
(0.006)\end{array}$ & $\begin{array}{l}-0.003 \\
(0.004)\end{array}$ & $\begin{array}{l}0.002 \\
(0.005)\end{array}$ & $\begin{array}{l}0.004 \\
(0.005)\end{array}$ \\
\hline Palermo. 1948 & $\begin{array}{l}-0.010 \\
(0.011)\end{array}$ & $\begin{array}{l}0.012 \\
(0.014)\end{array}$ & $\begin{array}{l}-0.002 \\
(0.002)\end{array}$ & $\begin{array}{l}-0.002 \\
(0.003)\end{array}$ & $\begin{array}{l}-0.003 \\
(0.002)\end{array}$ \\
\hline Palermo. 1949 & $\begin{array}{l}0.004 \\
(0.005)\end{array}$ & $\begin{array}{l}-0.014 \\
(0.017)\end{array}$ & $\begin{array}{l}0.008 \\
(0.011)\end{array}$ & $\begin{array}{l}0.004 \\
(0.006)\end{array}$ & $\begin{array}{l}0.002 \\
(0.005)\end{array}$ \\
\hline Palermo. 1950 & $\begin{array}{l}-0.008 \\
(0.008)\end{array}$ & $\begin{array}{l}0.007 \\
(0.009)\end{array}$ & $\begin{array}{l}-0.004 \\
(0.007)\end{array}$ & $\begin{array}{l}-0.005 \\
(0.007)\end{array}$ & $\begin{array}{l}-0.001 \\
(0.003)\end{array}$ \\
\hline Palermo· 1951 & $\begin{array}{l}0.015 \\
(0.016)\end{array}$ & $\begin{array}{l}-0.004 \\
(0.005)\end{array}$ & $\begin{array}{l}0.006 \\
(0.008)\end{array}$ & $\begin{array}{l}0.001 \\
(0.002)\end{array}$ & $\begin{array}{l}-0.004 \\
(0.006)\end{array}$ \\
\hline Observations & 6,238 & 6,238 & 6,238 & 6,238 & 6,238 \\
\hline$F$-statistic & 0.67 & 0.87 & 0.51 & 0.98 & 0.46 \\
\hline
\end{tabular}

Notes. OLS regressions predicting outcomes in the pre-Productivity Program period for 804 firms that applied for management transfer (Panel A), 1,178 firms that applied for technology transfer (Panel B), and 1,612 firms that applied for the combined management and technology transfers (Panel C). Thirty firms whose applications were rejected are excluded. Data are provided at the firm level. Standard errors are block-bootstrapped at the province level with 200 replications. Region x year dummies are included, but their coefficients are not reported. Monza, Vicenza, Pisa, Salerno, and Palermo are indicator variables for treatment provinces. All the dependent variables are expressed in logs. Employment is the number of employees per firm; Assets, Sales, and Value Added are in 2010 USD, reevaluated from 1951 to 2010 values at 1 lira $=30.884$ euros and exchanged at 0.780 euro $=$ USD 1 ; TFPR is the logarithm of total factor productivity revenue, estimated using the Ackerberg, Caves and Frazer (2006) method. The $F$ -statistics at the bottom of each panel test whether all the interaction terms between treatment provinces and the time trend are jointly zero. 
Table A.9-: Pre-Productivity Program Differences between Treated and Comparison Provinces, by Firm Application Date

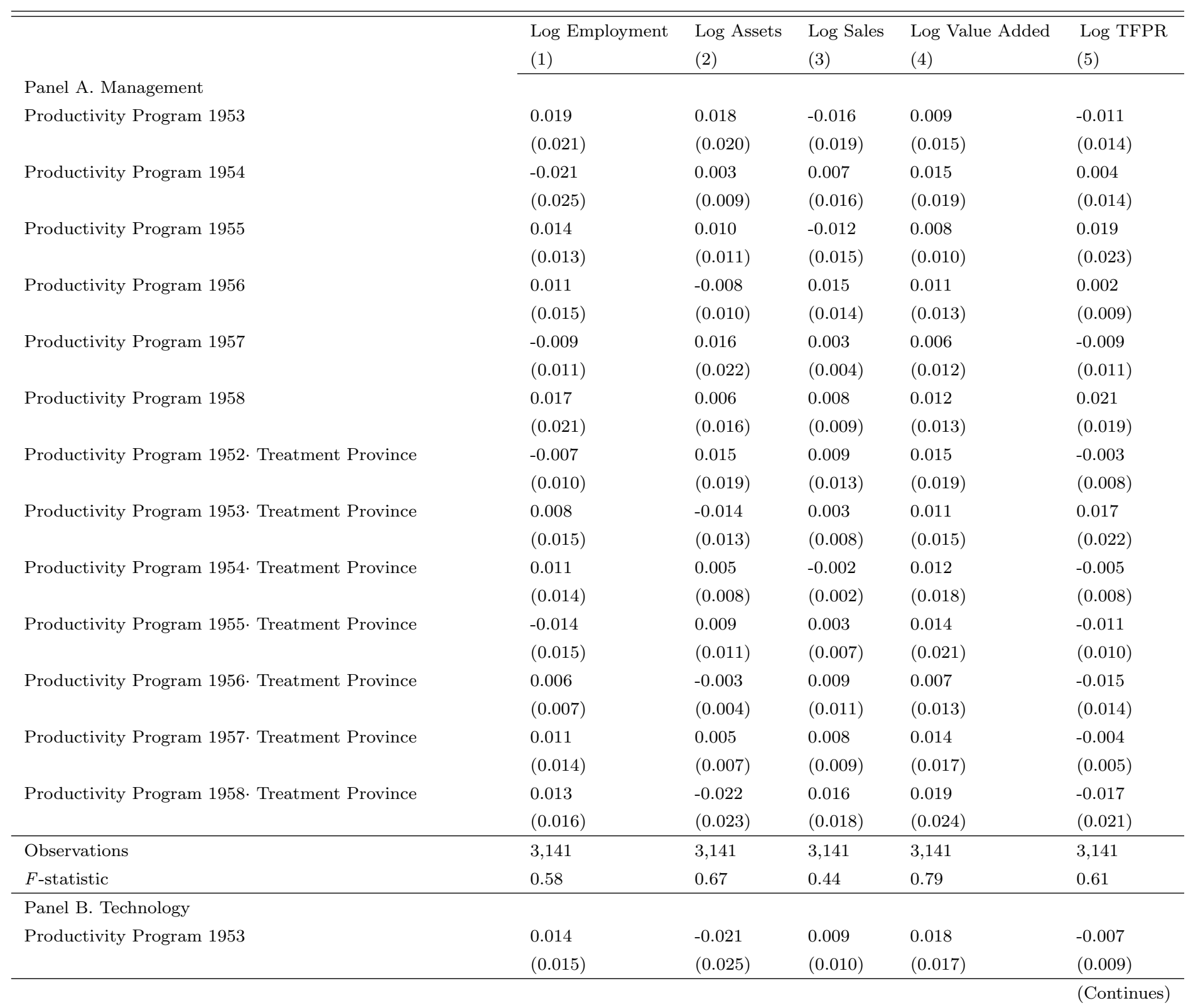


Table A.9-: Continued

\begin{tabular}{|c|c|c|c|c|c|}
\hline & $\begin{array}{l}\text { Log Employment } \\
\text { (1) }\end{array}$ & $\begin{array}{l}\text { Log Assets } \\
(2)\end{array}$ & $\begin{array}{l}\text { Log Sales } \\
(3)\end{array}$ & $\begin{array}{l}\text { Log Value Added } \\
(4)\end{array}$ & $\begin{array}{l}\text { Log TFPR } \\
(5)\end{array}$ \\
\hline \multirow[t]{2}{*}{ Productivity Program 1954} & 0.012 & 0.019 & -0.007 & 0.011 & -0.003 \\
\hline & $(0.015)$ & $(0.021)$ & $(0.011)$ & $(0.014)$ & $(0.006)$ \\
\hline \multirow[t]{2}{*}{ Productivity Program 1955} & 0.015 & 0.017 & -0.021 & 0.013 & 0.025 \\
\hline & $(0.021)$ & $(0.022)$ & $(0.025)$ & $(0.016)$ & $(0.031)$ \\
\hline \multirow[t]{2}{*}{ Productivity Program 1956} & -0.021 & 0.023 & 0.015 & 0.014 & 0.011 \\
\hline & $(0.028)$ & $(0.026)$ & $(0.019)$ & $(0.024)$ & $(0.014)$ \\
\hline \multirow[t]{2}{*}{ Productivity Program 1957} & 0.024 & -0.010 & -0.011 & 0.021 & 0.018 \\
\hline & $(0.023)$ & $(0.015)$ & $(0.013)$ & $(0.025)$ & $(0.022)$ \\
\hline \multirow[t]{2}{*}{ Productivity Program 1958} & 0.009 & 0.022 & 0.016 & -0.025 & 0.008 \\
\hline & $(0.010)$ & $(0.025)$ & $(0.015)$ & $(0.031)$ & $(0.018)$ \\
\hline \multirow[t]{2}{*}{ Productivity Program 1952. Treatment Province } & 0.013 & -0.016 & 0.012 & 0.009 & -0.017 \\
\hline & $(0.016)$ & $(0.021)$ & $(0.011)$ & $(0.009)$ & $(0.023)$ \\
\hline \multirow[t]{2}{*}{ Productivity Program 1953. Treatment Province } & -0.011 & 0.014 & -0.018 & 0.021 & 0.013 \\
\hline & $(0.021)$ & $(0.016)$ & $(0.023)$ & $(0.025)$ & $(0.014)$ \\
\hline \multirow[t]{2}{*}{ Productivity Program 1954. Treatment Province } & 0.008 & -0.017 & 0.014 & 0.016 & 0.011 \\
\hline & $(0.011)$ & $(0.024)$ & $(0.015)$ & $(0.023)$ & $(0.010)$ \\
\hline \multirow[t]{2}{*}{ Productivity Program 1955. Treatment Province } & 0.010 & 0.024 & 0.011 & 0.020 & 0.015 \\
\hline & $(0.012)$ & $(0.028)$ & $(0.013)$ & $(0.023)$ & $(0.018)$ \\
\hline \multirow[t]{2}{*}{ Productivity Program 1956. Treatment Province } & -0.015 & 0.013 & 0.021 & -0.016 & 0.019 \\
\hline & $(0.023)$ & $(0.018)$ & $(0.025)$ & $(0.019)$ & $(0.023)$ \\
\hline \multirow[t]{2}{*}{ Productivity Program 1957. Treatment Province } & 0.021 & -0.017 & -0.023 & 0.013 & 0.024 \\
\hline & $(0.023)$ & $(0.025)$ & $(0.026)$ & $(0.016)$ & $(0.029)$ \\
\hline \multirow[t]{2}{*}{ Productivity Program 1958. Treatment Province } & 0.016 & 0.019 & 0.010 & 0.014 & 0.008 \\
\hline & $(0.015)$ & $(0.022)$ & $(0.012)$ & $(0.018)$ & $(0.007)$ \\
\hline Observations & 4,678 & 4,678 & 4,678 & 4,678 & 4,678 \\
\hline$F$-statistic & 0.44 & 0.78 & 0.54 & 0.89 & 0.31 \\
\hline \multicolumn{6}{|l|}{ Panel C. Combined } \\
\hline \multirow[t]{2}{*}{ Productivity Program 1953} & 0.015 & 0.013 & -0.020 & 0.018 & 0.014 \\
\hline & $(0.021)$ & $(0.016)$ & $(0.019)$ & $(0.022)$ & $(0.017)$ \\
\hline \multirow[t]{2}{*}{ Productivity Program 1954} & 0.025 & -0.017 & 0.009 & 0.011 & 0.015 \\
\hline & $(0.029)$ & $(0.022)$ & $(0.011)$ & $(0.015)$ & $(0.019)$ \\
\hline
\end{tabular}


Table A.9-: Continued

\begin{tabular}{|c|c|c|c|c|c|}
\hline & $\begin{array}{l}\text { Log Employment } \\
\text { (1) }\end{array}$ & $\begin{array}{l}\text { Log Assets } \\
\text { (2) }\end{array}$ & $\begin{array}{l}\text { Log Sales } \\
(3)\end{array}$ & $\begin{array}{l}\text { Log Value Added } \\
(4)\end{array}$ & $\begin{array}{l}\text { Log TFPR } \\
\text { (5) }\end{array}$ \\
\hline \multirow[t]{2}{*}{ Productivity Program 1955} & 0.022 & -0.020 & 0.016 & 0.008 & -0.015 \\
\hline & $(0.025)$ & $(0.022)$ & $(0.019)$ & $(0.009)$ & $(0.019)$ \\
\hline \multirow[t]{2}{*}{ Productivity Program 1956} & 0.011 & -0.008 & 0.018 & -0.012 & 0.023 \\
\hline & $(0.013)$ & $(0.009)$ & $(0.025)$ & $(0.014)$ & $(0.028)$ \\
\hline \multirow[t]{2}{*}{ Productivity Program 1957} & 0.019 & 0.014 & -0.010 & 0.021 & 0.014 \\
\hline & $(0.023)$ & $(0.016)$ & $(0.016)$ & $(0.024)$ & $(0.013)$ \\
\hline \multirow[t]{2}{*}{ Productivity Program 1958} & 0.014 & -0.013 & 0.022 & 0.014 & 0.009 \\
\hline & $(0.018)$ & $(0.012)$ & $(0.023)$ & $(0.016)$ & $(0.008)$ \\
\hline \multirow[t]{2}{*}{ Productivity Program 1952. Treatment Province } & -0.017 & 0.013 & 0.018 & -0.021 & -0.024 \\
\hline & $(0.021)$ & $(0.017)$ & $(0.023)$ & $(0.25)$ & $(0.031)$ \\
\hline \multirow[t]{2}{*}{ Productivity Program 1953. Treatment Province } & -0.008 & 0.020 & 0.025 & -0.014 & 0.017 \\
\hline & $(0.009)$ & $(0.024)$ & $(0.031)$ & $(0.015)$ & $(0.024)$ \\
\hline \multirow[t]{2}{*}{ Productivity Program 1954. Treatment Province } & 0.023 & 0.011 & 0.020 & 0.008 & 0.013 \\
\hline & $(0.021)$ & $(0.015)$ & $(0.022)$ & $(0.011)$ & $(0.016)$ \\
\hline \multirow[t]{2}{*}{ Productivity Program 1955. Treatment Province } & -0.014 & 0.009 & -0.017 & 0.012 & 0.010 \\
\hline & $(0.019)$ & $(0.009)$ & $(0.021)$ & $(0.018)$ & $(0.009)$ \\
\hline \multirow[t]{2}{*}{ Productivity Program 1956. Treatment Province } & 0.011 & 0.017 & 0.020 & -0.009 & 0.021 \\
\hline & $(0.013)$ & $(0.023)$ & $(0.025)$ & $(0.011)$ & $(0.024)$ \\
\hline \multirow[t]{2}{*}{ Productivity Program 1957. Treatment Province } & -0.012 & 0.010 & 0.016 & 0.011 & 0.017 \\
\hline & $(0.015)$ & $(0.014)$ & $(0.022)$ & $(0.024)$ & $(0.021)$ \\
\hline \multirow[t]{2}{*}{ Productivity Program 1958. Treatment Province } & 0.013 & -0.008 & 0.019 & -0.013 & -0.007 \\
\hline & $(0.019)$ & $(0.010)$ & $(0.022)$ & $(0.015)$ & $(0.010)$ \\
\hline Observations & 6,238 & 6,238 & 6,238 & 6,238 & 6,238 \\
\hline$F$-statistic & 0.56 & 0.69 & 0.36 & 0.49 & 0.71 \\
\hline Pilot region & Yes & Yes & Yes & Yes & Yes \\
\hline Pilot region $\mathrm{x}$ time $\mathrm{FE}$ & No & No & No & No & No \\
\hline
\end{tabular}

Notes. Coefficients estimated from regressing each dependent variable on a full set of dummies for the year in which firms received/should have received the U.S. transfers and an interaction term between these dummies and an indicator for firms located in treatment provinces for 804 firms that applied for management transfer (Panel A), 1,178 firms that applied for technology transfer (Panel B), and 1,612 firms that applied for the combined management and technology transfers (Panel C). Thirty firms whose applications were rejected are excluded. The excluded year is 1952 . Standard errors are blockbootstrapped at province level with 200 replications. Employment is the total number of employees per firm; Assets, Sales, and Value Added are in 2010 USD, reevaluated from 1951 to 2010 values at 1 lira $=30.884$ euros and exchanged at 0.780 euro $=$ USD 1 ; TFPR is the logarithm of total factor productivity revenue, estimated using the Ackerberg, Caves and Frazer (2006) method. The $F$-statistics at the bottom of each panel test whether all the coefficients are jointly zero. 
Table A.10-: Pre-Productivity Program Differences in Time Trends between Treated and Comparison Provinces, in the Four Years before the Implementation of the Productivity Program

\begin{tabular}{|c|c|c|c|c|c|c|c|c|c|c|}
\hline & \multicolumn{2}{|c|}{ Log Employment } & \multicolumn{2}{|c|}{ Log Assets } & \multicolumn{2}{|c|}{ Log Sales } & \multicolumn{2}{|c|}{ Log Value Added } & \multicolumn{2}{|c|}{ Log TFPR } \\
\hline & $(1)$ & $(2)$ & (3) & $(4)$ & $(5)$ & $(6)$ & $(7)$ & $(8)$ & $(9)$ & $(10)$ \\
\hline \multicolumn{11}{|l|}{ Panel A. Management } \\
\hline \multirow[t]{2}{*}{ Treatment Province. $(\mathrm{t}-1)$} & 0.009 & 0.008 & 0.013 & 0.011 & 0.015 & 0.015 & 0.008 & 0.008 & 0.012 & 0.010 \\
\hline & $(0.011)$ & $(0.010)$ & $(0.015)$ & $(0.014)$ & $(0.019)$ & $(0.018)$ & $(0.010)$ & $(0.009)$ & $(0.015)$ & $(0.013)$ \\
\hline \multirow[t]{2}{*}{ Treatment Province $(\mathrm{t}-2)$} & 0.011 & 0.009 & -0.016 & -0.012 & 0.024 & 0.022 & -0.005 & -0.005 & 0.009 & 0.007 \\
\hline & $(0.012)$ & $(0.012)$ & $(0.019)$ & $(0.013)$ & $(0.029)$ & $(0.028)$ & $(0.006)$ & $(0.004)$ & $(0.012)$ & $(0.011)$ \\
\hline \multirow[t]{2}{*}{ Treatment Province $\cdot(t-3)$} & -0.010 & -0.010 & 0.022 & 0.020 & -0.013 & -0.012 & 0.006 & 0.005 & 0.009 & 0.08 \\
\hline & $(0.014)$ & $(0.012)$ & $(0.024)$ & $(0.021)$ & $(0.015)$ & $(0.014)$ & $(0.005)$ & $(0.005)$ & $(0.010)$ & $(0.009)$ \\
\hline \multirow[t]{2}{*}{ Treatment Province $\cdot(\mathrm{t}-4)$} & 0.016 & 0.013 & -0.012 & -0.011 & 0.013 & 0.011 & 0.011 & 0.010 & 0.021 & 0.018 \\
\hline & $(0.018)$ & $(0.015)$ & $(0.014)$ & $(0.013)$ & $(0.016)$ & $(0.015)$ & $(0.010)$ & $(0.008)$ & $(0.026)$ & $(0.024)$ \\
\hline Observations & 3,655 & 3,655 & 3,655 & 3,655 & 3,655 & 3,655 & 3,655 & 3,655 & 3,655 & 3,655 \\
\hline$F$-statistic & 0.77 & 0.85 & 0.59 & 0.63 & 0.42 & 0.49 & 0.55 & 0.62 & 0.59 & 0.71 \\
\hline \multicolumn{11}{|l|}{ Panel B. Technology } \\
\hline \multirow[t]{2}{*}{ Treatment Province $\cdot(\mathrm{t}-1)$} & 0.018 & 0.016 & 0.008 & 0.008 & 0.022 & 0.021 & 0.013 & 0.012 & 0.021 & 0.019 \\
\hline & $(0.022)$ & $(0.021)$ & $(0.009)$ & $(0.008)$ & $(0.026)$ & $(0.024)$ & $(0.018)$ & $(0.017)$ & $(0.024)$ & $(0.022)$ \\
\hline \multirow[t]{2}{*}{ Treatment Province. $(\mathrm{t}-2)$} & 0.012 & 0.012 & -0.019 & -0.015 & 0.011 & 0.009 & 0.020 & 0.018 & -0.024 & -0.021 \\
\hline & $(0.015)$ & $(0.013)$ & $(0.021)$ & $(0.020)$ & $(0.014)$ & $(0.012)$ & $(0.023)$ & $(0.022)$ & $(0.031)$ & $(0.027)$ \\
\hline \multirow[t]{2}{*}{ Treatment Province. $(\mathrm{t}-3)$} & 0.025 & 0.021 & 0.010 & 0.009 & 0.015 & 0.014 & 0.011 & 0.008 & 0.016 & 0.012 \\
\hline & $(0.031)$ & $(0.029)$ & $(0.011)$ & $(0.010)$ & $(0.019)$ & $(0.017)$ & $(0.014)$ & $(0.011)$ & $(0.019)$ & $(0.018)$ \\
\hline \multirow[t]{2}{*}{ Treatment Province. $(\mathrm{t}-4)$} & 0.017 & 0.016 & -0.021 & -0.019 & 0.008 & 0.006 & 0.018 & 0.018 & -0.009 & -0.006 \\
\hline & $(0.024)$ & $(0.022)$ & $(0.023)$ & $(0.020)$ & $(0.009)$ & $(0.008)$ & $(0.021)$ & $(0.021)$ & $(0.010)$ & $(0.008)$ \\
\hline Observations & 5,265 & 5,265 & 5,265 & 5,265 & 5,265 & 5,265 & 5,265 & 5,265 & 5,265 & 5,265 \\
\hline$F$-statistic & 0.89 & 0.91 & 0.45 & 0.61 & 0.56 & 0.73 & 0.42 & 0.56 & 0.69 & 0.78 \\
\hline
\end{tabular}


Table A.10-: Continued

\begin{tabular}{|c|c|c|c|c|c|c|c|c|c|c|}
\hline & \multicolumn{2}{|c|}{ Log Employment } & \multicolumn{2}{|c|}{ Log Assets } & \multicolumn{2}{|c|}{ Log Sales } & \multicolumn{2}{|c|}{ Log Value Added } & \multicolumn{2}{|c|}{ Log TFPR } \\
\hline & $(1)$ & $(2)$ & (3) & $(4)$ & $(5)$ & $(6)$ & $(7)$ & $(8)$ & (9) & $(10)$ \\
\hline \multicolumn{11}{|l|}{ Panel C. Combined } \\
\hline \multirow[t]{2}{*}{ Treatment Province $\cdot(\mathrm{t}-1)$} & 0.016 & 0.013 & 0.020 & 0.018 & 0.009 & 0.008 & -0.017 & -0.015 & 0.011 & 0.011 \\
\hline & $(0.022)$ & $(0.020)$ & $(0.027)$ & $(0.022)$ & $(0.010)$ & $(0.007)$ & $(0.025)$ & $(0.025)$ & $(0.014)$ & $(0.013)$ \\
\hline \multirow[t]{2}{*}{ Treatment Province $\cdot(\mathrm{t}-2)$} & 0.023 & 0.020 & 0.015 & 0.014 & -0.008 & -0.007 & 0.009 & 0.008 & 0.019 & 0.018 \\
\hline & $(0.026)$ & $(0.025)$ & $(0.016)$ & $(0.016)$ & $(0.012)$ & $(0.012)$ & $(0.008)$ & $(0.008)$ & $(0.022)$ & $(0.022)$ \\
\hline \multirow[t]{2}{*}{ Treatment Province $(\mathrm{t}-3)$} & -0.017 & -0.015 & 0.019 & 0.016 & 0.025 & 0.022 & 0.013 & 0.012 & -0.007 & -0.007 \\
\hline & $(0.020)$ & $(0.017)$ & $(0.025)$ & $(0.021)$ & $(0.029)$ & $(0.027)$ & $(0.018)$ & $(0.017)$ & $(0.009)$ & $(0.007)$ \\
\hline \multirow[t]{2}{*}{ Treatment Province $\cdot(\mathrm{t}-4)$} & 0.008 & 0.007 & 0.023 & 0.019 & -0.011 & -0.010 & 0.018 & 0.014 & 0.023 & 0.022 \\
\hline & $(0.007)$ & $(0.007)$ & $(0.028)$ & $(0.027)$ & $(0.015)$ & $(0.014)$ & $(0.022)$ & $(0.019)$ & $(0.032)$ & $(0.029)$ \\
\hline Observations & 7,340 & 7,340 & 7,340 & 7,340 & 7,340 & 7,340 & 7,340 & 7,340 & 7,340 & 7,340 \\
\hline$F$-statistic & 0.59 & 0.73 & 0.67 & 0.91 & 0.56 & 0.69 & 0.071 & 0.98 & 0.43 & 0.55 \\
\hline Pilot region & Yes & Yes & Yes & Yes & Yes & Yes & Yes & Yes & Yes & Yes \\
\hline Pilot region $\mathrm{x}$ time $\mathrm{FE}$ & No & Yes & No & Yes & No & Yes & No & Yes & No & Yes \\
\hline
\end{tabular}

Notes. OLS regressions predicting outcomes in the pre-Productivity Program period for 731 firms that applied for management transfer (Panel A), 1,053 firms that applied for technology transfer (Panel B), and 1,468 firms that applied for the combined management and technology transfers (Panel C). The sample is restricted to firms that survived until the intervention year. Data are provided at the firm level. The trend is allowed to vary freely for each year before the implementation of the Productivity Program. Year dummies are included, but their coefficients are not reported. Standard errors are block-bootstrapped at the province level with 200 replications. Employment is the total number of employees per firm; Assets, Sales, and Value Added are in 2010 USD, reevaluated from 1951 to 2010 values at 1 lira $=30.884$ euros and exchanged at 0.780 euro $=$ USD 1 ; TFPR is the logarithm of total factor productivity revenue, estimated using the Ackerberg, Caves and Frazer (2006) method. The $F$-statistics at the bottom of each panel test whether all the interaction terms between treatment provinces and the year dummy variables are jointly zero. 
Table A.11—: Firms That Exited the Market before Implementation of the Productivity Program

\begin{tabular}{|c|c|c|c|c|c|c|c|c|c|}
\hline & \multicolumn{3}{|c|}{ A. Management } & \multicolumn{3}{|c|}{ B. Technology } & \multicolumn{3}{|c|}{ C. Combined } \\
\hline & \multicolumn{2}{|c|}{ Treatment Provinces } & \multirow{2}{*}{ Diff $p$-value } & \multicolumn{2}{|c|}{ Treatment Provinces } & \multirow{2}{*}{ Diff $p$-value } & \multicolumn{2}{|c|}{ Treatment Provinces } & \multirow{2}{*}{ Diff $p$-value } \\
\hline & Yes & No & & Yes & No & & Yes & No & \\
\hline & Mean & Mean & & Mean & Mean & & Mean & Mean & \\
\hline & $(1)$ & $(2)$ & $(3)$ & $(4)$ & $(5)$ & $(6)$ & $(7)$ & $(8)$ & (9) \\
\hline Plants per firm & 1.12 & 1.15 & 0.809 & 1.33 & 1.29 & 0.432 & 1.21 & 1.28 & 0.340 \\
\hline Employees per firm & 39.85 & 37.65 & 0.567 & 34.51 & 38.95 & 0.489 & 33.45 & 31.21 & 0.435 \\
\hline Current assets ( $\mathrm{k}$ in 2010 USD) & $405,671.33$ & $420,983.12$ & 0.482 & $567,893.36$ & $542,142.59$ & 0.501 & $606,093.23$ & $587,784.30$ & 0.483 \\
\hline Annual sales (k in 2010 USD) & 203,567 & $234,402.34$ & 0.453 & $245,682.32$ & $287,671.11$ & 0.444 & $324,591.34$ & $301,298.35$ & 0.348 \\
\hline Value added ( $\mathrm{k}$ in $2010 \mathrm{USD}$ ) & 80.94 & 85.93 & 0.521 & 90.83 & 94.84 & 0.536 & 101.34 & 106.79 & 0.210 \\
\hline Age & 11.23 & 12.56 & 0.322 & 10.09 & 11.38 & 0.439 & 12.37 & 10.76 & 0.398 \\
\hline Productivity (log TFPR) & 2.02 & 2.05 & 0.492 & 2.12 & 2.10 & 0.321 & 2.09 & 2.14 & 0.394 \\
\hline Export & 0.11 & 0.13 & 0.671 & 0.11 & 0.10 & 0.702 & 0.09 & 0.11 & 0.475 \\
\hline Family-managed & 0.55 & 0.57 & 0.459 & 0.52 & 0.56 & 0.540 & 0.55 & 0.51 & 0.555 \\
\hline $\mathrm{N}$ & 15 & 58 & & 18 & 107 & & 44 & 100 & \\
\hline Ratio (\%) & 10.27 & 8.81 & & 7.73 & 11.32 & & 11.40 & 8.15 & \\
\hline
\end{tabular}

Notes. Balancing tests for firms that closed down before the implementation of the Productivity Program. Data are provided at the firm level. Columns $1,2,4,5,7$, and 8 report the mean, respectively, in treatment and comparison provinces. Columns 3, 6 , and 9 report the $p$-value of the mean difference. Plants per firm reports the number of plants per firm; Employees per firm reports the number of employees per firm; Current assets ( $k$ in 2010 USD), Annual sales ( $k$ in 2010 USD), and Value added ( $k$ in 2010 USD) are in 2010 USD, reevaluated from 1951 to 2010 values at 1 lira=30.884 euros and exchanged at 0.780 euro=USD 1; Productivity $(\log T F P R)$ is the logarithm of firm productivity, estimated using the Ackerberg, Caves and Frazer (2006) method; Export and Family-managed are indicator variables that equal one if, respectively, a firm exported and was family-managed. 
Table A.12-: Cox Survival Model Estimation of Firm Shutdown Hazard

\begin{tabular}{|c|c|c|c|c|c|c|c|c|}
\hline & \multicolumn{8}{|c|}{ Shut-Down Hazard Ratio } \\
\hline & \multicolumn{4}{|c|}{ Proportional hazard ratio $(1-4)$} & \multicolumn{4}{|c|}{ Different hazard ratio for $t \geq 7(5-8)$} \\
\hline & (1) & $(2)$ & (3) & $(4)$ & (5) & $(6)$ & $(7)$ & $(8)$ \\
\hline \multicolumn{9}{|l|}{ A. Management } \\
\hline \multirow[t]{2}{*}{ Treatment Province } & 0.294 & 0.292 & 0.289 & 0.276 & 0.621 & 0.620 & 0.618 & 0.615 \\
\hline & $(0.085)$ & $(0.084)$ & $(0.080)$ & $(0.079)$ & $(0.148)$ & $(0.146)$ & $(0.143)$ & $(0.138)$ \\
\hline \multirow[t]{2}{*}{ Treatment Province, $t \geq 7$} & & & & & 0.413 & 0.409 & 0.404 & 0.401 \\
\hline & & & & & $(0.132)$ & $(0.130)$ & $(0.127)$ & $(0.126)$ \\
\hline Observations & 731 & 731 & 731 & 731 & 731 & 731 & 731 & 731 \\
\hline Failures & 193 & 193 & 193 & 193 & 193 & 193 & 193 & 193 \\
\hline \multicolumn{9}{|l|}{ B. Technology } \\
\hline \multirow[t]{2}{*}{ Treatment Province } & 0.407 & 0.404 & 0.399 & 0.388 & 0.723 & 0.721 & 0.717 & 0.715 \\
\hline & $(0.076)$ & $(0.074)$ & $(0.071)$ & $(0.068)$ & $(0.155)$ & $(0.151)$ & $(0.149)$ & $(0.145)$ \\
\hline \multirow[t]{2}{*}{ Treatment Province, $t \geq 7$} & & & & & 0.591 & 0.589 & 0.585 & 0.581 \\
\hline & & & & & $(0.132)$ & $(0.129)$ & $(0.125)$ & $(0.123)$ \\
\hline Observations & 1,035 & 1,035 & 1,035 & 1,035 & 1,035 & 1,035 & 1,035 & 1,035 \\
\hline Failures & 305 & 305 & 305 & 305 & 305 & 305 & 305 & 305 \\
\hline \multicolumn{9}{|l|}{ C. Combined } \\
\hline \multirow[t]{2}{*}{ Treatment Province } & 0.163 & 0.160 & 0.157 & 0.151 & 0.744 & 0.739 & 0.734 & 0.729 \\
\hline & $(0.037)$ & $(0.033)$ & $(0.030)$ & $(0.025)$ & $(0.031)$ & $(0.030)$ & $(0.028)$ & $(0.026)$ \\
\hline \multirow[t]{2}{*}{ Treatment Province, $t \geq 7$} & & & & & 0.311 & 0.308 & 0.302 & 0.298 \\
\hline & & & & & $(0.025)$ & $(0.021)$ & $(0.020)$ & $(0.018)$ \\
\hline Observations & 1,468 & 1,468 & 1,468 & 1,468 & 1,468 & 1,468 & 1,468 & 1,468 \\
\hline Failures & 386 & 386 & 386 & 386 & 386 & 386 & 386 & 386 \\
\hline Pilot region controls & Yes & Yes & Yes & Yes & Yes & Yes & Yes & Yes \\
\hline Calendar year controls & No & Yes & Yes & Yes & No & Yes & Yes & Yes \\
\hline Industry controls & No & No & Yes & Yes & No & No & Yes & Yes \\
\hline Pre-Productivity Program controls & No & No & No & Yes & No & No & No & Yes \\
\hline
\end{tabular}

Notes. Shutdown hazard ratio estimated from the Cox survival model $h(t)=h_{0}(t) \exp \left(\beta \operatorname{Exp}_{\operatorname{Prov}}+\lambda_{r}\right)$, where $h(t)$ is the hazard of shutdown $t$ years after the U.S. intervention, Treated Province $i$ is an indicator variable for firms located in treatment provinces, and $\lambda_{r}$ is pilot region fixed effects, for 731 firms that applied for management transfer (Panel A), 1,053 firms that applied for technology transfer (Panel B), and and 1,468 firms that applied for the combined management and technology transfers (Panel C). Data are provided at firm level. Columns 1-4 report estimates of a proportional hazard ratio, constant over time; columns 5-8 report estimates in which the hazard ratio is allowed to change seven years after the Productivity Program. 
Table A.13-: Sales, Employment, and TFPR Growth Rates in Italy and in Firms Eligible for the Productivity Program, 1950-1970

\begin{tabular}{lcllll}
\hline \hline & $\begin{array}{l}\text { Italy } \\
(1)\end{array}$ & $\begin{array}{l}\text { Management } \\
(2)\end{array}$ & $\begin{array}{l}\text { Technology } \\
(3)\end{array}$ & $\begin{array}{l}\text { Combined } \\
(4)\end{array}$ & $\begin{array}{l}\text { Did Not Apply } \\
(5)\end{array}$ \\
\cline { 2 - 6 } Real GDP/Sales & & & & \\
1950-1955 & 6.45 & 4.79 & 4.23 & 4.98 & 4.21 \\
$1955-1960$ & 5.23 & 4.51 & 4.12 & 4.73 & 4.04 \\
$1960-1965$ & 6.37 & 4.23 & 3.08 & 4.21 & 2.99 \\
$1965-1970$ & 5.80 & 3.23 & 2.96 & 3.45 & 2.54 \\
\hline Employment & & & & & \\
$1950-1955$ & 3.49 & 3.55 & 3.12 & 4.30 & 3.07 \\
$1955-1960$ & 2.12 & 3.21 & 3.07 & 3.59 & 2.49 \\
$1960-1965$ & 2.00 & 2.99 & 2.78 & 3.01 & 1.95 \\
$1965-1970$ & 1.95 & 2.08 & 2.43 & 2.21 & 1.97 \\
\hline TFPR & & & & & \\
$1950-1955$ & 3.57 & 3.55 & 2.41 & 3.78 & 2.02 \\
$1955-1960$ & 2.94 & 2.45 & 2.03 & 2.98 & 1.80 \\
$1960-1965$ & 2.49 & 2.33 & 1.98 & 2.57 & 1.55 \\
$1965-1970$ & 1.97 & 2.14 & 1.82 & 2.27 & 1.55 \\
\hline \hline
\end{tabular}

Notes. Average annual growth rates (\%) of Italian real GDP and firm Sales, and Employment and TFPR of all Italian manufacturing firms; of eligible firms that applied for the management transfer, the technology transfer, and the combined management and technology transfers and did not receive U.S. assistance; and of eligible firms that did not apply between 1950-1955, 1955-1960, 1960-1965, 1965-1970. Italian growth rates are from the Historical Archive of the Bank of Italy (ASBI), accessed in February 2014. 
Table A.14-: Lee's Tightened Bounds

\begin{tabular}{|c|c|c|c|c|c|c|c|c|c|}
\hline & \multicolumn{3}{|c|}{ A. Management } & \multicolumn{3}{|c|}{ B. Technology } & \multicolumn{3}{|c|}{ C. Combined } \\
\hline & $\begin{array}{l}\text { Log Sales } \\
(1)\end{array}$ & $\begin{array}{l}\text { Log Employees } \\
(2)\end{array}$ & $\begin{array}{l}\text { Log TFPR } \\
(3)\end{array}$ & $\begin{array}{l}\text { Log Sales } \\
(4)\end{array}$ & $\begin{array}{l}\text { Log Employees } \\
(5)\end{array}$ & $\begin{array}{l}\text { Log TFPR } \\
(6)\end{array}$ & $\begin{array}{l}\text { Log Sales } \\
(7)\end{array}$ & $\begin{array}{l}\text { Log Employees } \\
(8)\end{array}$ & $\begin{array}{l}\text { Log TFPR } \\
(9)\end{array}$ \\
\hline Upper Bound Year1 & $\begin{array}{l}0.065 \\
(0.015)\end{array}$ & $\begin{array}{l}0.015 \\
(0.010)\end{array}$ & $\begin{array}{l}0.115 \\
(0.034)\end{array}$ & $\begin{array}{l}0.009 \\
(0.010)\end{array}$ & $\begin{array}{l}0.020 \\
(0.014)\end{array}$ & $\begin{array}{l}0.022 \\
(0.024)\end{array}$ & $\begin{array}{l}0.099 \\
(0.017)\end{array}$ & $\begin{array}{l}0.046 \\
(0.015)\end{array}$ & $\begin{array}{l}0.207 \\
(0.034)\end{array}$ \\
\hline Upper Bound Year5 & $\begin{array}{l}0.126 \\
(0.029)\end{array}$ & $\begin{array}{l}0.079 \\
(0.022)\end{array}$ & $\begin{array}{l}0.207 \\
(0.067)\end{array}$ & $\begin{array}{l}0.054 \\
(0.020)\end{array}$ & $\begin{array}{l}0.047 \\
(0.022)\end{array}$ & $\begin{array}{l}0.092 \\
(0.031)\end{array}$ & $\begin{array}{l}0.287 \\
(0.067)\end{array}$ & $\begin{array}{l}0.168 \\
(0.056)\end{array}$ & $\begin{array}{l}0.367 \\
(0.078)\end{array}$ \\
\hline Upper Bound Year10 & $\begin{array}{l}0.229 \\
(0.042)\end{array}$ & $\begin{array}{l}0.255 \\
(0.060)\end{array}$ & $\begin{array}{l}0.289 \\
(0.084)\end{array}$ & $\begin{array}{l}0.087 \\
(0.036)\end{array}$ & $\begin{array}{l}0.099 \\
(0.044)\end{array}$ & $\begin{array}{l}0.130 \\
(0.061)\end{array}$ & $\begin{array}{l}0.374 \\
(0.098)\end{array}$ & $\begin{array}{l}0.371 \\
(0.084)\end{array}$ & $\begin{array}{l}0.519 \\
(0.089)\end{array}$ \\
\hline Upper Bound Year15 & $\begin{array}{l}0.382 \\
(0.089)\end{array}$ & $\begin{array}{l}0.359 \\
(0.101)\end{array}$ & $\begin{array}{l}0.399 \\
(0.098)\end{array}$ & $\begin{array}{l}0.084 \\
(0.039)\end{array}$ & $\begin{array}{l}0.095 \\
(0.048)\end{array}$ & $\begin{array}{l}0.128 \\
(0.061)\end{array}$ & $\begin{array}{l}0.511 \\
(0.103)\end{array}$ & $\begin{array}{l}0.525 \\
(0.116)\end{array}$ & $\begin{array}{l}0.682 \\
(0.109)\end{array}$ \\
\hline Observations & 13,902 & 13,902 & 13,902 & 20,213 & 20,213 & 20,213 & 27,870 & 27,870 & 27,870 \\
\hline Number of firms & 731 & 731 & 731 & 1,053 & 1,053 & 1,053 & 1,468 & 1,468 & 1,468 \\
\hline
\end{tabular}

Notes. Lee (2009)'s tightened bounds calculated for coefficients from equation 1 for firms that applied for management transfer (Panel A), firms that applied for technology transfer (Panel B), and firms that applied for the combined management and technology transfers (Panel C). Data are provided at the firm level. The dependent variables are logged deflated Sales, converted from 1951 Italian lira to 2010 euro and exchanged at 0.780 euro $=$ USD 1 (columns 1, 4, and 7); logged Employees, reporting the number of employees per firm (columns 2, 5, and 8); and logged TFPR, estimated using the Ackerberg, Caves and Frazer (2006) method (columns 3, 6, and 9). Standard errors are block-bootstrapped at the province level with 200 replications. 
Table A.15-: Comparison of the Effects of the Productivity Program over Time

\begin{tabular}{|c|c|c|c|c|c|c|}
\hline & \multicolumn{2}{|c|}{ Log Sales (1-2) } & \multicolumn{2}{|c|}{ Log Employment (3-4) } & \multicolumn{2}{|c|}{ Log TFPR (5-6) } \\
\hline & (1) & $(2)$ & (3) & (4) & $(5)$ & (6) \\
\hline \multicolumn{7}{|l|}{ A. Management } \\
\hline Year1 = Year5 & 2.98 & 2.77 & 3.41 & 2.85 & 2.91 & 3.11 \\
\hline Year5 = Year10 & 3.01 & 2.93 & 2.65 & 2.77 & 2.74 & 2.61 \\
\hline Year10 = Year15 & 2.68 & 2.82 & 2.89 & 2.91 & 2.67 & 2.92 \\
\hline \multicolumn{7}{|l|}{ B. Technology } \\
\hline Year1 = Year5 & 2.65 & 2.83 & 2.98 & 3.01 & 2.76 & 2.67 \\
\hline Year5 = Year10 & 2.42 & 2.24 & 2.11 & 2.45 & 2.37 & 2.38 \\
\hline Year10 = Year15 & 1.13 & 1.08 & 1.45 & 1.22 & 1.37 & 1.19 \\
\hline \multicolumn{7}{|l|}{ C. Combined } \\
\hline Year1 = Year5 & 2.65 & 2.92 & 2.97 & 2.94 & 2.76 & 2.95 \\
\hline Year5 = Year10 & 2.72 & 2.88 & 2.61 & 2.89 & 2.89 & 2.80 \\
\hline Year10 = Year15 & 2.76 & 2.67 & 2.67 & 2.78 & 2.98 & 3.04 \\
\hline \multicolumn{7}{|l|}{ D. Management $=$ Technology } \\
\hline Year1 Management = Technology & & 16.35 & & 13.58 & & 17.46 \\
\hline Year5 Management = Technology & & 17.60 & & 12.77 & & 16.90 \\
\hline Year10 Management = Technology & & 15.59 & & 13.89 & & 12.32 \\
\hline Year15 Management = Technology & & 13.29 & & 16.54 & & 18.55 \\
\hline \multicolumn{7}{|l|}{ E. Comparison across Transfers } \\
\hline Year1 Combined = Mgmt.+Tech. & & 17.56 & & 15.91 & & 18.29 \\
\hline Year5 Combined = Mgmt.+Tech. & & 12.77 & & 14.66 & & 17.72 \\
\hline Year10 Combined = Mgmt. + Tech. & & 16.39 & & 17.62 & & 18.66 \\
\hline Year15 Combined = Mgmt.+Tech. & & 13.36 & & 16.32 & & 15.32 \\
\hline Sample & Balanced & Matched & Balanced & Matched & Balanced & Matched \\
\hline Firm FE & Yes & Yes & Yes & Yes & Yes & Yes \\
\hline Year FE & Yes & Yes & Yes & Yes & Yes & Yes \\
\hline
\end{tabular}

Notes. Panels A-C report the $t$-tests of the null hypothesis of equality of the coefficients estimated from equation 1 between one and five, five and ten, and ten and fifteen years after the Productivity Program, respectively, for firms that applied for management transfer (Panel A), firms that applied for technology transfer (Panel B), and firms that applied for the combined management and technology transfers (Panel C). Panels D and E report, respectively, the $F$-statistics of the null hypothesis of equality between the coefficients on management and technology transfers, and between the coefficients on the combined management and technology transfers and the sum of coefficients on management and technology transfers one, five, ten, and fifteen years after the Productivity Program, estimated from equation 2. Data are provided at the firm level. The dependent variables are logged deflated Sales converted from 1951 Italian lira to 2010 euro and exchanged at 0.780 euro $=$ USD 1 (columns 1-2); logged Employment, reporting the number of employees per firm (columns 3-4); and logged TFPR, estimated using the Ackerberg, Caves and Frazer (2006) method (columns 5-6). 
Table A.16-: Heterogeneity Effects: by Productivity Levels

\begin{tabular}{|c|c|c|c|c|c|c|c|c|c|}
\hline & \multicolumn{3}{|c|}{ A. Management } & \multicolumn{3}{|c|}{ B. Technology } & \multicolumn{3}{|c|}{ C. Combined } \\
\hline & $\begin{array}{l}\text { Log Sales } \\
(1)\end{array}$ & $\begin{array}{l}\text { Log Employees } \\
(2)\end{array}$ & $\begin{array}{l}\text { Log TFPR } \\
(3)\end{array}$ & $\begin{array}{l}\text { Log Sales } \\
(4)\end{array}$ & $\begin{array}{l}\text { Log Employees } \\
\text { (5) }\end{array}$ & $\begin{array}{l}\text { Log TFPR } \\
(6)\end{array}$ & $\begin{array}{l}\text { Log Sales } \\
\text { (7) }\end{array}$ & $\begin{array}{l}\text { Log Employees } \\
\text { (8) }\end{array}$ & $\begin{array}{l}\text { Log TFPR } \\
(9)\end{array}$ \\
\hline \multicolumn{10}{|c|}{ I. Below National Industry Mean } \\
\hline \multirow[t]{2}{*}{ Year1AfterPP } & 0.065 & 0.010 & 0.152 & 0.005 & 0.008 & 0.015 & 0.105 & 0.053 & 0.217 \\
\hline & $(0.017)$ & $(0.008)$ & $(0.025)$ & $(0.004)$ & $(0.009)$ & $(0.013)$ & $(0.027)$ & $(0.012)$ & $(0.022)$ \\
\hline \multirow[t]{2}{*}{ Year15AfterPP } & 0.367 & 0.337 & 0.443 & 0.051 & 0.055 & 0.083 & 0.483 & 0.529 & 0.651 \\
\hline & $(0.062)$ & $(0.071)$ & $(0.068)$ & $(0.030)$ & $(0.030)$ & $(0.042)$ & $(0.073)$ & $(0.081)$ & $(0.092)$ \\
\hline \multicolumn{10}{|c|}{ II. Above National Industry Mean } \\
\hline \multirow[t]{2}{*}{ Year1AfterPP } & 0.047 & 0.005 & 0.135 & 0.010 & 0.018 & 0.027 & 0.079 & 0.029 & 0.186 \\
\hline & $(0.015)$ & $(0.006)$ & $(0.034)$ & $(0.006)$ & $(0.014)$ & $(0.012)$ & $(0.021)$ & $(0.007)$ & $(0.053)$ \\
\hline \multirow[t]{2}{*}{ Year15AfterPP } & 0.341 & 0.288 & 0.386 & 0.082 & 0.095 & 0.121 & 0.378 & 0.479 & 0.574 \\
\hline & $(0.067)$ & $(0.073)$ & $(0.081)$ & $(0.025)$ & $(0.027)$ & $(0.036)$ & $(0.068)$ & $(0.073)$ & $(0.087)$ \\
\hline Sample & Balanced & Balanced & Balanced & Balanced & Balanced & Balanced & Balanced & Balanced & Balanced \\
\hline Firm FE & Yes & Yes & Yes & Yes & Yes & Yes & Yes & Yes & Yes \\
\hline Year FE & Yes & Yes & Yes & Yes & Yes & Yes & Yes & Yes & Yes \\
\hline
\end{tabular}

Notes. OLS estimation of equation 1 for 538 firms that chose management transfer (columns 1-3), 748 firms that chose technology transfer (columns 4-6), and 1,082 firms that chose the combined management and technology transfers (columns 7-9) and survived in the 15 years after the Productivity Program, stratifying the sample by firm productivity level compared to the national industry average. Industries below the national mean are food, wood, and minerals. Industries above the national mean are textile, machinery, and chemicals. The dependent variables are logged (deflated) Sales converted from 1951 Italian lira to 2010 euro and exchanged at 0.780 euro = USD 1 (columns 1, 4, and 7); logged Employees, reporting the number of employees per firm (columns 2, 5, and 8); and logged TFPR, estimated using the Ackerberg, Caves and Frazer (2006) method (columns 3, 6, and 9). Data are provided at the firm level. Standard errors are block-bootstrapped at the province level with 200 replications. 
Table A.17-: Heterogeneity Effects: by 1951 Firm Size

\begin{tabular}{|c|c|c|c|c|c|c|c|c|c|}
\hline & \multicolumn{3}{|c|}{ A. Management } & \multicolumn{3}{|c|}{ B. Technology } & \multicolumn{3}{|c|}{ C. Combined } \\
\hline & $\begin{array}{l}\text { Log Sales } \\
\text { (1) }\end{array}$ & $\begin{array}{l}\text { Log Employees } \\
\text { (2) }\end{array}$ & $\begin{array}{l}\text { Log TFPR } \\
\text { (3) }\end{array}$ & $\begin{array}{l}\text { Log Sales } \\
(4)\end{array}$ & $\begin{array}{l}\text { Log Employees } \\
\text { (5) }\end{array}$ & $\begin{array}{l}\text { Log TFPR } \\
\text { (6) }\end{array}$ & $\begin{array}{l}\text { Log Sales } \\
(7)\end{array}$ & $\begin{array}{l}\text { Log Employees } \\
\text { (8) }\end{array}$ & $\begin{array}{l}\text { Log TFPR } \\
(9)\end{array}$ \\
\hline \multicolumn{10}{|c|}{ I. Fewer than 30 employees } \\
\hline Year1AfterPP & $\begin{array}{l}0.040 \\
(0.019)\end{array}$ & $\begin{array}{l}0.006 \\
(0.007)\end{array}$ & $\begin{array}{l}0.103 \\
(0.027)\end{array}$ & $\begin{array}{l}0.005 \\
(0.007)\end{array}$ & $\begin{array}{l}0.006 \\
(0.005)\end{array}$ & $\begin{array}{l}0.012 \\
(0.015)\end{array}$ & $\begin{array}{l}0.072 \\
(0.019)\end{array}$ & $\begin{array}{l}0.031 \\
(0.007)\end{array}$ & $\begin{array}{l}0.189 \\
(0.029)\end{array}$ \\
\hline Year15AfterPP & $\begin{array}{l}0.389 \\
(0.073)\end{array}$ & $\begin{array}{l}0.345 \\
(0.065)\end{array}$ & $\begin{array}{l}0.441 \\
(0.072)\end{array}$ & $\begin{array}{l}0.048 \\
(0.025)\end{array}$ & $\begin{array}{l}0.057 \\
(0.029)\end{array}$ & $\begin{array}{l}0.094 \\
(0.041)\end{array}$ & $\begin{array}{l}0.484 \\
(0.081)\end{array}$ & $\begin{array}{l}0.525 \\
(0.087)\end{array}$ & $\begin{array}{l}0.678 \\
(0.097)\end{array}$ \\
\hline \multicolumn{10}{|c|}{ II. 30-49 employees } \\
\hline Year1AfterPP & $\begin{array}{l}0.041 \\
(0.020)\end{array}$ & $\begin{array}{l}0.005 \\
(0.006)\end{array}$ & $\begin{array}{l}0.125 \\
(0.031)\end{array}$ & $\begin{array}{l}0.004 \\
(0.005)\end{array}$ & $\begin{array}{l}0.008 \\
(0.009)\end{array}$ & $\begin{array}{l}0.014 \\
(0.015)\end{array}$ & $\begin{array}{l}0.075 \\
(0.020)\end{array}$ & $\begin{array}{l}0.024 \\
(0.006)\end{array}$ & $\begin{array}{l}0.177 \\
(0.032)\end{array}$ \\
\hline Year15AfterPP & $\begin{array}{l}0.361 \\
(0.078)\end{array}$ & $\begin{array}{l}0.322 \\
(0.062)\end{array}$ & $\begin{array}{l}0.433 \\
(0.078)\end{array}$ & $\begin{array}{l}0.057 \\
(0.027)\end{array}$ & $\begin{array}{l}0.062 \\
(0.032)\end{array}$ & $\begin{array}{l}0.099 \\
(0.044)\end{array}$ & $\begin{array}{l}0.431 \\
(0.097)\end{array}$ & $\begin{array}{l}0.505 \\
(0.086)\end{array}$ & $\begin{array}{l}0.663 \\
(0.092)\end{array}$ \\
\hline \multicolumn{10}{|c|}{ III. 50-99 employees } \\
\hline Year1AfterPP & $\begin{array}{l}0.063 \\
(0.023)\end{array}$ & $\begin{array}{l}0.010 \\
(0.009)\end{array}$ & $\begin{array}{l}0.153 \\
(0.035)\end{array}$ & $\begin{array}{l}0.010 \\
(0.006)\end{array}$ & $\begin{array}{l}0.016 \\
(0.010)\end{array}$ & $\begin{array}{l}0.023 \\
(0.013)\end{array}$ & $\begin{array}{l}0.095 \\
(0.021)\end{array}$ & $\begin{array}{l}0.043 \\
(0.012)\end{array}$ & $\begin{array}{l}0.209 \\
(0.035)\end{array}$ \\
\hline \multicolumn{10}{|c|}{ IV. 100 employees or more } \\
\hline Year1AfterPP & $\begin{array}{l}0.078 \\
(0.027)\end{array}$ & $\begin{array}{l}0.013 \\
(0.008)\end{array}$ & $\begin{array}{l}0.161 \\
(0.037)\end{array}$ & $\begin{array}{l}0.016 \\
(0.009)\end{array}$ & $\begin{array}{l}0.019 \\
(0.016)\end{array}$ & $\begin{array}{l}0.025 \\
(0.015)\end{array}$ & $\begin{array}{l}0.108 \\
(0.025)\end{array}$ & $\begin{array}{l}0.051 \\
(0.017)\end{array}$ & $\begin{array}{l}0.219 \\
(0.038)\end{array}$ \\
\hline Year15AfterPP & $\begin{array}{l}0.212 \\
(0.079)\end{array}$ & $\begin{array}{l}0.249 \\
(0.065)\end{array}$ & $\begin{array}{l}0.300 \\
(0.073)\end{array}$ & $\begin{array}{l}0.082 \\
(0.026)\end{array}$ & $\begin{array}{l}0.091 \\
(0.032)\end{array}$ & $\begin{array}{l}0.125 \\
(0.035)\end{array}$ & $\begin{array}{l}0.395 \\
(0.091)\end{array}$ & $\begin{array}{l}0.442 \\
(0.083)\end{array}$ & $\begin{array}{l}0.531 \\
(0.089)\end{array}$ \\
\hline Sample & Balanced & Balanced & Balanced & Balanced & Balanced & Balanced & Balanced & Balanced & Balanced \\
\hline Firm FE & Yes & Yes & Yes & Yes & Yes & Yes & Yes & Yes & Yes \\
\hline Year FE & Yes & Yes & Yes & Yes & Yes & Yes & Yes & Yes & Yes \\
\hline
\end{tabular}

Notes. OLS estimation of equation 1 for 538 firms that chose management transfer (columns 1-3), 748 firms that chose technology transfer (columns 4-6), and 1,082 firms that chose the combined management and technology transfers (columns 7-9) and survived in the 15 years after the Productivity Program, stratifying the sample by firm size. The dependent variables are logged (deflated) Sales converted from 1951 Italian lira to 2010 euro and exchanged at 0.780 euro = USD 1 (columns 1, 4, and 7); logged Employees, reporting the number of employees per firm (columns 2,5, and 8); and logged TFPR, estimated using the Ackerberg, Caves and Frazer (2006) method (columns 3, 6, and 9). Data are provided at the firm level. Standard errors are block-bootstrapped at the province level with 200 replications. 
Table A.18-: Heterogeneity Effects: by Region

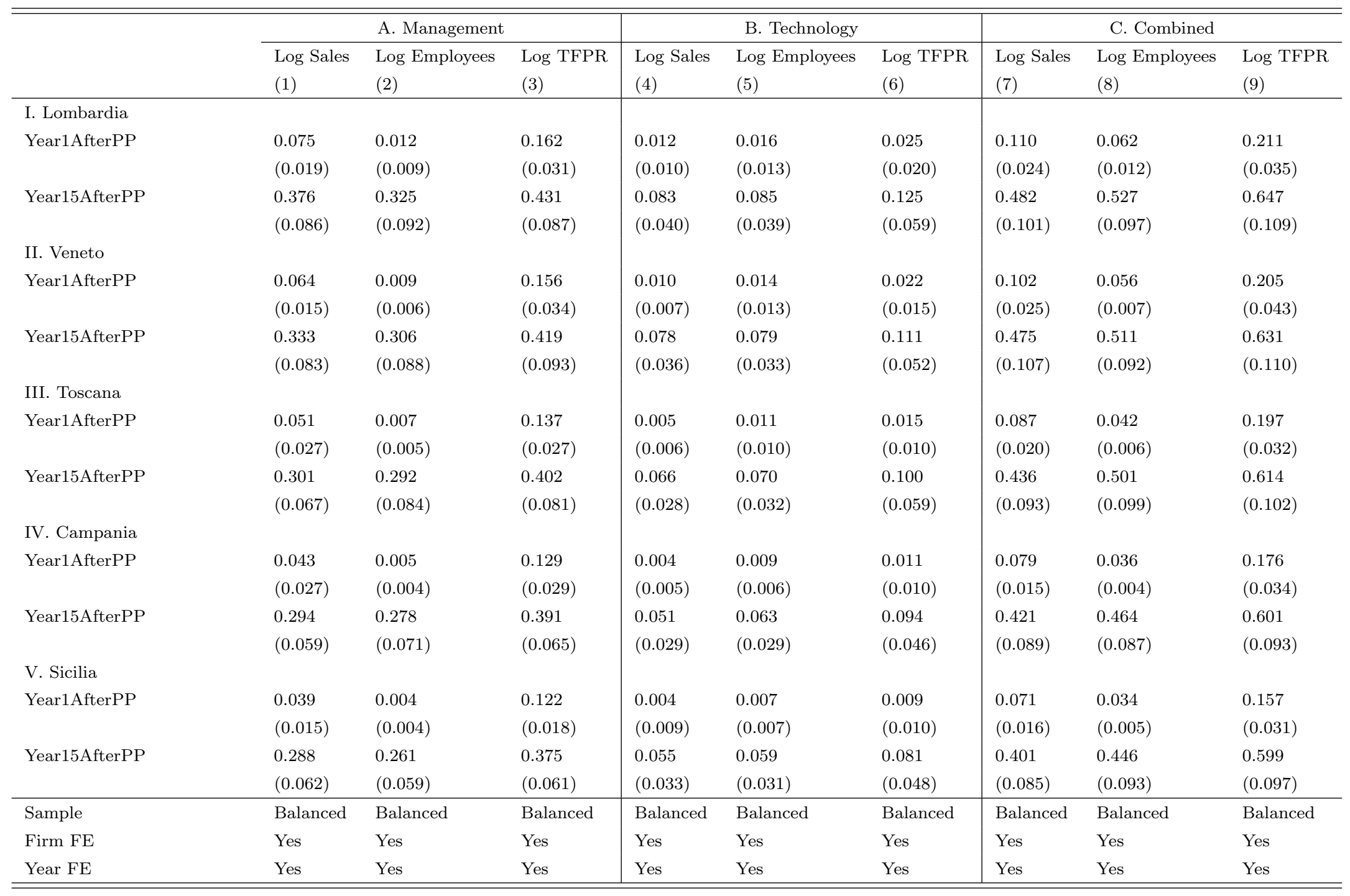

Notes. OLS estimation of eq. 1 for 538 firms that chose management transfer (columns 1-3), 748 firms that chose tech transfer (columns 4-6), and 1,082 firms that chose the combined transfers (columns 7-9) and survived in the 15 years after the Productivity Program, stratifying the sample by region. The dependent variables are logged (deflated) Sales converted from 1951 Italian lira to 2010 euro and exchanged at 0.780 euro=USD 1 (columns 1, 4, and 7);

logged Employees, reporting the number of employees per firm (columns 2, 5, and 8); and logged TFPR, estimated using the Ackerberg, Caves and Frazer (2006) method (columns 3, 6, and 9). Data are provided at the firm level. Std. errors are block-bootstrapped at the province level with 200 replications. 
Table A.19-: Heterogeneity Effects: by Industry Growth Rate

\begin{tabular}{|c|c|c|c|c|c|c|c|c|c|}
\hline & \multicolumn{3}{|c|}{ A. Management } & \multicolumn{3}{|c|}{ B. Technology } & \multicolumn{3}{|c|}{ C. Combined } \\
\hline & $\begin{array}{l}\text { Log Sales } \\
\text { (1) }\end{array}$ & $\begin{array}{l}\text { Log Employees } \\
\text { (2) }\end{array}$ & $\begin{array}{l}\text { Log TFPR } \\
\text { (3) }\end{array}$ & $\begin{array}{l}\text { Log Sales } \\
\text { (4) }\end{array}$ & $\begin{array}{l}\text { Log Employees } \\
\text { (5) }\end{array}$ & $\begin{array}{l}\text { Log TFPR } \\
(6)\end{array}$ & $\begin{array}{l}\text { Log Sales } \\
\text { (7) }\end{array}$ & $\begin{array}{l}\text { Log Employees } \\
\text { (8) }\end{array}$ & $\begin{array}{l}\text { Log TFPR } \\
\text { (9) }\end{array}$ \\
\hline \multicolumn{10}{|c|}{ I. Above the mean } \\
\hline \multirow[t]{2}{*}{ Year1AfterPP } & 0.047 & 0.005 & 0.129 & 0.008 & 0.017 & 0.013 & 0.086 & 0.036 & 0.181 \\
\hline & $(0.021)$ & $(0.005)$ & $(0.029)$ & $(0.006)$ & $(0.015)$ & $(0.012)$ & $(0.019)$ & $(0.007)$ & $(0.029)$ \\
\hline \multirow[t]{2}{*}{ Year15AfterPP } & 0.300 & 0.307 & 0.434 & 0.059 & 0.085 & 0.115 & 0.493 & 0.519 & 0.636 \\
\hline & $(0.064)$ & $(0.078)$ & $(0.085)$ & $(0.029)$ & $(0.036)$ & $(0.048)$ & $(0.108)$ & $(0.097)$ & $(0.088)$ \\
\hline \multicolumn{10}{|c|}{ II. Below the mean } \\
\hline \multirow[t]{2}{*}{ Year1AfterPP } & 0.064 & 0.010 & 0.152 & 0.006 & 0.011 & 0.024 & 0.095 & 0.047 & 0.213 \\
\hline & $(0.023)$ & $(0.007)$ & $(0.028)$ & $(0.007)$ & $(0.014)$ & $(0.020)$ & $(0.019)$ & $(0.011)$ & $(0.049)$ \\
\hline \multirow[t]{2}{*}{ Year15AfterPP } & 0.369 & 0.295 & 0.388 & 0.079 & 0.065 & 0.089 & 0.394 & 0.477 & 0.609 \\
\hline & $(0.066)$ & $(0.087)$ & $(0.093)$ & $(0.034)$ & $(0.032)$ & $(0.044)$ & $(0.103)$ & $(0.099)$ & $(0.110)$ \\
\hline Sample & Balanced & Balanced & Balanced & Balanced & Balanced & Balanced & Balanced & Balanced & Balanced \\
\hline Firm FE & Yes & Yes & Yes & Yes & Yes & Yes & Yes & Yes & Yes \\
\hline
\end{tabular}

Notes. OLS estimation of equation 1 for 538 firms that chose management transfer (columns 1-3), 748 firms that chose technology transfer (columns 4-6), and 1,082 firms that chose the combined management and technology transfers (columns 7-9) and survived in the 15 years after the Productivity Program, stratifying the sample by industry growth rate. The dependent variables are logged (deflated) Sales converted from 1951 Italian lira to 2010 euro and exchanged at 0.780 euro $=$ USD 1 (columns 1, 4, and 7); logged Employees, reporting the number of employees per firm (columns 2, 5, and 8); and logged TFPR, estimated using the Ackerberg, Caves and Frazer (2006) method (columns 3, 6, and 9). Data are provided at the firm level. Standard errors are block-bootstrapped at the province level with 200 replications. 
Table A.20-: Heterogeneity Effects: by Year of Participation in the Productivity Program

\begin{tabular}{|c|c|c|c|c|c|c|c|c|c|}
\hline & \multicolumn{3}{|c|}{ A. Management } & \multicolumn{3}{|c|}{ B. Technology } & \multicolumn{3}{|c|}{ C. Combined } \\
\hline & $\begin{array}{l}\text { Log Sales } \\
(1)\end{array}$ & $\begin{array}{l}\text { Log Employees } \\
(2)\end{array}$ & $\begin{array}{l}\text { Log TFPR } \\
(3)\end{array}$ & $\begin{array}{l}\text { Log Sales } \\
(4)\end{array}$ & $\begin{array}{l}\text { Log Employees } \\
(5)\end{array}$ & $\begin{array}{l}\text { Log TFPR } \\
(6)\end{array}$ & $\begin{array}{l}\text { Log Sales } \\
(7)\end{array}$ & $\begin{array}{l}\text { Log Employees } \\
(8)\end{array}$ & $\begin{array}{l}\text { Log TFPR } \\
(9)\end{array}$ \\
\hline \multicolumn{10}{|l|}{ I. 1952} \\
\hline Year1AfterPP & $\begin{array}{l}0.060 \\
(0.020)\end{array}$ & $\begin{array}{l}0.008 \\
(0.008)\end{array}$ & $\begin{array}{l}0.142 \\
(0.030)\end{array}$ & $\begin{array}{l}0.009 \\
(0.010)\end{array}$ & $\begin{array}{l}0.016 \\
(0.013)\end{array}$ & $\begin{array}{l}0.024 \\
(0.015)\end{array}$ & $\begin{array}{l}0.065 \\
(0.020)\end{array}$ & $\begin{array}{l}0.038 \\
(0.011)\end{array}$ & $\begin{array}{l}0.195 \\
(0.034)\end{array}$ \\
\hline Year15AfterPP & $\begin{array}{l}0.335 \\
(0.062)\end{array}$ & $\begin{array}{l}0.306 \\
(0.089)\end{array}$ & $\begin{array}{l}0.401 \\
(0.091)\end{array}$ & $\begin{array}{l}0.063 \\
(0.025)\end{array}$ & $\begin{array}{l}0.077 \\
(0.036)\end{array}$ & $\begin{array}{l}0.105 \\
(0.046)\end{array}$ & $\begin{array}{l}0.454 \\
(0.094)\end{array}$ & $\begin{array}{l}0.495 \\
(0.099)\end{array}$ & $\begin{array}{l}0.628 \\
(0.103)\end{array}$ \\
\hline \multicolumn{10}{|l|}{ II. 1953} \\
\hline Year1AfterPP & $\begin{array}{l}0.061 \\
(0.015)\end{array}$ & $\begin{array}{l}0.009 \\
(0.008)\end{array}$ & $\begin{array}{l}0.139 \\
(0.034)\end{array}$ & $\begin{array}{l}0.005 \\
(0.006)\end{array}$ & $\begin{array}{l}0.014 \\
(0.017)\end{array}$ & $\begin{array}{l}0.017 \\
(0.019)\end{array}$ & $\begin{array}{l}0.067 \\
(0.013)\end{array}$ & $\begin{array}{l}0.035 \\
(0.008)\end{array}$ & $\begin{array}{l}0.198 \\
(0.037)\end{array}$ \\
\hline Year15AfterPP & $\begin{array}{l}0.333 \\
(0.071)\end{array}$ & $\begin{array}{l}0.301 \\
(0.065)\end{array}$ & $\begin{array}{l}0.409 \\
(0.068)\end{array}$ & $\begin{array}{l}0.071 \\
(0.035)\end{array}$ & $\begin{array}{l}0.082 \\
(0.040)\end{array}$ & $\begin{array}{l}0.109 \\
(0.050)\end{array}$ & $\begin{array}{l}0.456 \\
(0.088)\end{array}$ & $\begin{array}{l}0.499 \\
(0.091)\end{array}$ & $\begin{array}{l}0.623 \\
(0.094)\end{array}$ \\
\hline \multicolumn{10}{|l|}{ III. 1954} \\
\hline Year1AfterPP & $\begin{array}{l}0.059 \\
(0.022)\end{array}$ & $\begin{array}{l}0.011 \\
(0.009)\end{array}$ & $\begin{array}{l}0.141 \\
(0.034)\end{array}$ & $\begin{array}{l}0.007 \\
(0.009)\end{array}$ & $\begin{array}{l}0.011 \\
(0.012)\end{array}$ & $\begin{array}{l}0.021 \\
(0.016)\end{array}$ & $\begin{array}{l}0.071 \\
(0.017)\end{array}$ & $\begin{array}{l}0.042 \\
(0.008)\end{array}$ & $\begin{array}{l}0.199 \\
(0.029)\end{array}$ \\
\hline \multicolumn{10}{|l|}{ IV. 1955} \\
\hline Year1AfterPP & $\begin{array}{l}0.058 \\
(0.015)\end{array}$ & $\begin{array}{l}0.012 \\
(0.008)\end{array}$ & $\begin{array}{l}0.138 \\
(0.024)\end{array}$ & $\begin{array}{l}0.008 \\
(0.009)\end{array}$ & $\begin{array}{l}0.012 \\
(0.014)\end{array}$ & $\begin{array}{l}0.016 \\
(0.011)\end{array}$ & $\begin{array}{l}0.072 \\
(0.013)\end{array}$ & $\begin{array}{l}0.043 \\
(0.010)\end{array}$ & $\begin{array}{l}0.191 \\
(0.054)\end{array}$ \\
\hline Year15AfterPP & $\begin{array}{l}0.335 \\
(0.049)\end{array}$ & $\begin{array}{l}0.309 \\
(0.056)\end{array}$ & $\begin{array}{l}0.411 \\
(0.052)\end{array}$ & $\begin{array}{l}0.066 \\
(0.036)\end{array}$ & $\begin{array}{l}0.078 \\
(0.039)\end{array}$ & $\begin{array}{l}0.111 \\
(0.049)\end{array}$ & $\begin{array}{l}0.456 \\
(0.108)\end{array}$ & $\begin{array}{l}0.494 \\
(0.089)\end{array}$ & $\begin{array}{l}0.619 \\
(0.104)\end{array}$ \\
\hline \multicolumn{10}{|l|}{ V. 1956} \\
\hline Year1AfterPP & $\begin{array}{l}0.057 \\
(0.016)\end{array}$ & $\begin{array}{l}0.009 \\
(0.006)\end{array}$ & $\begin{array}{l}0.140 \\
(0.033)\end{array}$ & $\begin{array}{l}0.009 \\
(0.009)\end{array}$ & $\begin{array}{l}0.019 \\
(0.017)\end{array}$ & $\begin{array}{l}0.020 \\
(0.010)\end{array}$ & $\begin{array}{l}0.068 \\
(0.017)\end{array}$ & $\begin{array}{l}0.044 \\
(0.007)\end{array}$ & $\begin{array}{l}0.197 \\
(0.029)\end{array}$ \\
\hline Year15AfterPP & $\begin{array}{l}0.334 \\
(0.081)\end{array}$ & $\begin{array}{l}0.295 \\
(0.079)\end{array}$ & $\begin{array}{l}0.395 \\
(0.088)\end{array}$ & $\begin{array}{l}0.072 \\
(0.039)\end{array}$ & $\begin{array}{l}0.081 \\
(0.042) \\
\end{array}$ & $\begin{array}{l}0.112 \\
(0.049)\end{array}$ & $\begin{array}{l}0.458 \\
(0.092)\end{array}$ & $\begin{array}{l}0.496 \\
(0.086)\end{array}$ & $\begin{array}{l}0.617 \\
(0.109)\end{array}$ \\
\hline $\begin{array}{l}\text { Sample } \\
\text { Firm FE }\end{array}$ & $\begin{array}{l}\text { Balanced } \\
\text { Yes }\end{array}$ & $\begin{array}{l}\text { Balanced } \\
\text { Yes }\end{array}$ & $\begin{array}{l}\text { Balanced } \\
\text { Yes }\end{array}$ & $\begin{array}{l}\text { Balanced } \\
\text { Yes }\end{array}$ & $\begin{array}{l}\text { Balanced } \\
\text { Yes }\end{array}$ & $\begin{array}{l}\text { Balanced } \\
\text { Yes }\end{array}$ & $\begin{array}{l}\text { Balanced } \\
\text { Yes }\end{array}$ & $\begin{array}{l}\text { Balanced } \\
\text { Yes }\end{array}$ & $\begin{array}{l}\text { Balanced } \\
\text { Yes }\end{array}$ \\
\hline
\end{tabular}


Table A.20-: Continued

\begin{tabular}{|c|c|c|c|c|c|c|c|c|c|}
\hline & \multicolumn{3}{|c|}{ A. Management } & \multicolumn{3}{|c|}{ B. Technology } & \multicolumn{3}{|c|}{ C. Combined } \\
\hline & $\begin{array}{l}\text { Log Sales } \\
(1)\end{array}$ & $\begin{array}{l}\text { Log Employees } \\
\text { (2) }\end{array}$ & $\begin{array}{l}\text { Log TFPR } \\
\text { (3) }\end{array}$ & $\begin{array}{l}\text { Log Sales } \\
(4)\end{array}$ & $\begin{array}{l}\text { Log Employees } \\
\text { (5) }\end{array}$ & $\begin{array}{l}\text { Log TFPR } \\
(6)\end{array}$ & $\begin{array}{l}\text { Log Sales } \\
(7)\end{array}$ & $\begin{array}{l}\text { Log Employees } \\
(8)\end{array}$ & $\begin{array}{l}\text { Log TFPR } \\
\text { (9) }\end{array}$ \\
\hline \multicolumn{10}{|l|}{ VI. 1957} \\
\hline Year1AfterPP & $\begin{array}{l}0.061 \\
(0.021)\end{array}$ & $\begin{array}{l}0.008 \\
(0.005)\end{array}$ & $\begin{array}{l}0.142 \\
(0.034)\end{array}$ & $\begin{array}{l}0.010 \\
(0.008)\end{array}$ & $\begin{array}{l}0.018 \\
(0.015)\end{array}$ & $\begin{array}{l}0.018 \\
(0.014)\end{array}$ & $\begin{array}{l}0.073 \\
(0.020)\end{array}$ & $\begin{array}{l}0.038 \\
(0.007)\end{array}$ & $\begin{array}{l}0.195 \\
(0.039)\end{array}$ \\
\hline Year15AfterPP & $\begin{array}{l}0.339 \\
(0.087)\end{array}$ & $\begin{array}{l}0.299 \\
(0.092)\end{array}$ & $\begin{array}{l}0.408 \\
(0.099)\end{array}$ & $\begin{array}{l}0.063 \\
(0.029)\end{array}$ & $\begin{array}{l}0.082 \\
(0.042)\end{array}$ & $\begin{array}{l}0.107 \\
(0.045)\end{array}$ & $\begin{array}{l}0.452 \\
(0.088)\end{array}$ & $\begin{array}{l}0.498 \\
(0.099)\end{array}$ & $\begin{array}{l}0.619 \\
(0.112)\end{array}$ \\
\hline \multicolumn{10}{|l|}{ VII. 1958} \\
\hline Year1AfterPP & $\begin{array}{l}0.060 \\
(0.023)\end{array}$ & $\begin{array}{l}0.009 \\
(0.008)\end{array}$ & $\begin{array}{l}0.141 \\
(0.032)\end{array}$ & $\begin{array}{l}0.009 \\
(0.006)\end{array}$ & $\begin{array}{l}0.013 \\
(0.015)\end{array}$ & $\begin{array}{l}0.019 \\
(0.012)\end{array}$ & $\begin{array}{l}0.071 \\
(0.019)\end{array}$ & $\begin{array}{l}0.036 \\
(0.007)\end{array}$ & $\begin{array}{l}0.193 \\
(0.029)\end{array}$ \\
\hline Year15AfterPP & $\begin{array}{l}0.338 \\
(0.066) \\
\end{array}$ & $\begin{array}{l}0.301 \\
(0.076) \\
\end{array}$ & $\begin{array}{l}0.399 \\
(0.078) \\
\end{array}$ & $\begin{array}{l}0.068 \\
(0.033) \\
\end{array}$ & $\begin{array}{l}0.078 \\
(0.035) \\
\end{array}$ & $\begin{array}{l}0.105 \\
(0.056) \\
\end{array}$ & $\begin{array}{l}0.451 \\
(0.112) \\
\end{array}$ & $\begin{array}{l}0.499 \\
(0.103) \\
\end{array}$ & $\begin{array}{l}0.620 \\
(0.129) \\
\end{array}$ \\
\hline Sample & Balanced & Balanced & Balanced & Balanced & Balanced & Balanced & Balanced & Balanced & Balanced \\
\hline Firm FE & Yes & Yes & Yes & Yes & Yes & Yes & Yes & Yes & Yes \\
\hline Year FE & Yes & Yes & Yes & Yes & Yes & Yes & Yes & Yes & Yes \\
\hline
\end{tabular}

Notes. OLS estimation of equation 1 for 538 firms that chose management transfer (columns 1-3), 748 firms that chose technology transfer (columns 4-6), and 1,082 firms that chose the combined management and technology transfers (columns 7-9) and survived in the 15 years after the Productivity Program, stratifying the sample by the year of participation in the Productivity Program. The dependent variables are logged (deflated) Sales converted from 1951 Italian lira to 2010 euro and exchanged at 0.780 euro = USD 1 (columns 1, 4, and 7); logged Employees, reporting the number of employees per firm (columns 2, 5, and 8); and logged TFPR, estimated using the Ackerberg, Caves and Frazer (2006) method (columns 3,6, and 9). Data are provided at the firm level. Standard errors are block-bootstrapped at the province level with 200 replications. 
Table A.21-: Effects of the Productivity Program on Exports and Imports

\begin{tabular}{|c|c|c|c|c|}
\hline & \multicolumn{2}{|c|}{ Exports } & \multicolumn{2}{|c|}{ Imports } \\
\hline & $\begin{array}{l}\text { Prob(Export) } \\
\text { (1) }\end{array}$ & $\begin{array}{l}\text { Exports } \\
(2)\end{array}$ & $\begin{array}{l}\text { Prob(Import) } \\
(3)\end{array}$ & $\begin{array}{l}\text { Log } \frac{\text { Imports }}{\text { Inputs }} \\
\text { (4) }\end{array}$ \\
\hline \multicolumn{5}{|l|}{ A. Management } \\
\hline Year1AfterPP & $\begin{array}{l}0.024 \\
(0.009)\end{array}$ & $\begin{array}{l}0.015 \\
(0.004)\end{array}$ & $\begin{array}{l}0.011 \\
(0.004)\end{array}$ & $\begin{array}{l}0.005 \\
(0.008)\end{array}$ \\
\hline Year5AfterPP & $\begin{array}{l}0.155 \\
(0.026)\end{array}$ & $\begin{array}{l}0.075 \\
(0.014)\end{array}$ & $\begin{array}{l}0.084 \\
(0.031)\end{array}$ & $\begin{array}{l}0.022 \\
(0.011)\end{array}$ \\
\hline Year10AfterPP & $\begin{array}{l}0.221 \\
(0.039)\end{array}$ & $\begin{array}{l}0.121 \\
(0.044)\end{array}$ & $\begin{array}{l}0.096 \\
(0.033)\end{array}$ & $\begin{array}{l}0.045 \\
(0.017)\end{array}$ \\
\hline Year15AfterPP & $\begin{array}{l}0.290 \\
(0.044)\end{array}$ & $\begin{array}{l}0.155 \\
(0.051)\end{array}$ & $\begin{array}{l}0.151 \\
(0.049)\end{array}$ & $\begin{array}{l}0.074 \\
(0.022)\end{array}$ \\
\hline Observations & 10,760 & 1,400 & 10,760 & 2,160 \\
\hline Number of firms & 538 & 70 & 538 & 108 \\
\hline \multicolumn{5}{|l|}{ B. Technology } \\
\hline Year1AfterPP & $\begin{array}{l}0.013 \\
(0.006)\end{array}$ & $\begin{array}{l}0.004 \\
(0.006)\end{array}$ & $\begin{array}{l}0.008 \\
(0.006)\end{array}$ & $\begin{array}{l}0.002 \\
(0.003)\end{array}$ \\
\hline Year5AfterPP & $\begin{array}{l}0.026 \\
(0.012)\end{array}$ & $\begin{array}{l}0.046 \\
(0.053)\end{array}$ & $\begin{array}{l}0.011 \\
(0.008)\end{array}$ & $\begin{array}{l}0.015 \\
(0.009)\end{array}$ \\
\hline Year10AfterPP & $\begin{array}{l}0.047 \\
(0.008)\end{array}$ & $\begin{array}{l}0.037 \\
(0.044)\end{array}$ & $\begin{array}{l}0.017 \\
(0.010)\end{array}$ & $\begin{array}{l}0.028 \\
(0.014)\end{array}$ \\
\hline Year15AfterPP & $\begin{array}{l}0.051 \\
(0.013)\end{array}$ & $\begin{array}{l}0.041 \\
(0.047)\end{array}$ & $\begin{array}{l}0.022 \\
(0.013)\end{array}$ & $\begin{array}{l}0.033 \\
(0.018)\end{array}$ \\
\hline Observations & 14,960 & 1,800 & 14,960 & 3,280 \\
\hline Number of firms & 748 & 90 & 748 & 164 \\
\hline C. Combined & & & & \\
\hline Year1AfterPP & $\begin{array}{l}0.033 \\
(0.010)\end{array}$ & $\begin{array}{l}0.045 \\
(0.011)\end{array}$ & $\begin{array}{l}0.016 \\
(0.006)\end{array}$ & $\begin{array}{l}0.029 \\
(0.012)\end{array}$ \\
\hline Year5AfterPP & $\begin{array}{l}0.172 \\
(0.031)\end{array}$ & $\begin{array}{l}0.122 \\
(0.031)\end{array}$ & $\begin{array}{l}0.093 \\
(0.031)\end{array}$ & $\begin{array}{l}0.044 \\
(0.016)\end{array}$ \\
\hline Year10AfterPP & $\begin{array}{l}0.275 \\
(0.041)\end{array}$ & $\begin{array}{l}0.156 \\
(0.036)\end{array}$ & $\begin{array}{l}0.105 \\
(0.037)\end{array}$ & $\begin{array}{l}0.059 \\
(0.020)\end{array}$ \\
\hline Year15AfterPP & $\begin{array}{l}0.315 \\
(0.056)\end{array}$ & $\begin{array}{l}0.191 \\
(0.041)\end{array}$ & $\begin{array}{l}0.162 \\
(0.045)\end{array}$ & $\begin{array}{l}0.098 \\
(0.033)\end{array}$ \\
\hline Observations & 21,640 & 3,020 & 21,640 & 1,840 \\
\hline Number of firms & 1,082 & 151 & 1,082 & 92 \\
\hline Firm FE & Yes & Yes & Yes & Yes \\
\hline Year FE & Yes & Yes & Yes & Yes \\
\hline
\end{tabular}

Notes. Coefficients estimated for 538 firms that applied for management transfer (Panel A), 748 firms that applied for technology transfer (Panel B), and 1,082 firms that applied for the combined management and technology transfers (Panel C) and survived in the 15 years after the Productivity Program. Columns 2 and 4 report the coefficients estimated from equation 1 for firms that were, respectively, exporters and importers in 1951. The dependent variables are Prob(Export), an indicator variable that equals one if a firm exported; logged deflated Exports converted from 1951 Italian lira to 2010 euro and exchanged at 0.780 euro=USD 1; Prob(Import), an indicator variable that equals one if a firm imported; and logged Imports/Inputs, converted from 1951 Italian lira to 2010 euro and exchanged at 0.780 euro $=$ USD 1. Standard errors are block-bootstrapped at the province level with 200 replications. 
Table A.22-: Effects of the Productivity Program on Firms That Did Not Export

\begin{tabular}{|c|c|c|c|}
\hline & $\begin{array}{l}\text { Log Sales } \\
\text { (1) }\end{array}$ & $\begin{array}{l}\text { Log Employment } \\
(2)\end{array}$ & $\begin{array}{l}\text { Log TFPR } \\
(3)\end{array}$ \\
\hline \multicolumn{4}{|l|}{ A. Management } \\
\hline \multirow[t]{2}{*}{ Year1AfterPP } & 0.049 & 0.005 & 0.095 \\
\hline & $(0.013)$ & $(0.009)$ & $(0.020)$ \\
\hline \multirow[t]{2}{*}{ Year5AfterPP } & 0.087 & 0.047 & 0.165 \\
\hline & $(0.020)$ & $(0.016)$ & $(0.021)$ \\
\hline \multirow[t]{2}{*}{ Year10AfterPP } & 0.122 & 0.194 & 0.232 \\
\hline & $(0.028)$ & $(0.028)$ & $(0.031)$ \\
\hline \multirow[t]{2}{*}{ Year15AfterPP } & 0.211 & 0.287 & 0.302 \\
\hline & $(0.035)$ & $(0.037)$ & $(0.041)$ \\
\hline Number of firms & 175 & 175 & 175 \\
\hline Observations & 3,500 & 3,500 & 3,500 \\
\hline \multicolumn{4}{|l|}{ B. Technology } \\
\hline \multirow[t]{2}{*}{ Year1AfterPP } & 0.005 & 0.007 & 0.013 \\
\hline & $(0.004)$ & $(0.010)$ & $(0.011)$ \\
\hline \multirow[t]{2}{*}{ Year5AfterPP } & 0.034 & 0.025 & 0.062 \\
\hline & $(0.013)$ & $(0.011)$ & $(0.015)$ \\
\hline \multirow[t]{2}{*}{ Year10AfterPP } & 0.062 & 0.067 & 0.094 \\
\hline & $(0.030)$ & $(0.027)$ & $(0.031)$ \\
\hline \multirow[t]{2}{*}{ Year15AfterPP } & 0.059 & 0.070 & 0.089 \\
\hline & $(0.028)$ & $(0.033)$ & $(0.036)$ \\
\hline Number of firms & 362 & 362 & 362 \\
\hline Observations & 7,240 & 7,240 & 7,240 \\
\hline \multicolumn{4}{|l|}{ C. Combined } \\
\hline \multirow[t]{2}{*}{ Year1AfterPP } & 0.065 & 0.024 & 0.151 \\
\hline & $(0.021)$ & $(0.009)$ & $(0.022)$ \\
\hline \multirow[t]{2}{*}{ Year5AfterPP } & 0.186 & 0.137 & 0.265 \\
\hline & $(0.024)$ & $(0.022)$ & $(0.029)$ \\
\hline \multirow[t]{2}{*}{ Year10AfterPP } & 0.211 & 0.212 & 0.346 \\
\hline & $(0.030)$ & $(0.028)$ & $(0.036)$ \\
\hline \multirow[t]{2}{*}{ Year15AfterPP } & 0.376 & 0.326 & 0.421 \\
\hline & $(0.033)$ & $(0.043)$ & $(0.051)$ \\
\hline Number of firms & 368 & 368 & 368 \\
\hline Observations & 7,360 & 7,360 & 7,360 \\
\hline Firm FE & Yes & Yes & Yes \\
\hline Year FE & Yes & Yes & Yes \\
\hline
\end{tabular}

Notes: Coefficients estimated from equation 1 for 175 firms that applied for management transfer (Panel A), 362 firms that applied for technology transfer (Panel B), and 368 firms that applied for both transfers (Panel C) that did not start exporting after the Productivity Program. Data are provided at the firm level. The dependent variables are logged deflated Sales converted from 1951 Italian lira to 2010 euro and exchanged at 0.780 euro = USD 1; logged Employment, reporting the number of employees per firm; and logged TFPR, estimated using the Ackerberg, Caves and Frazer (2006) method. Standard errors are block-bootstrapped at the province level with 200 replications. 
Table A.23-: Rate of Adoption of U.S. Managerial Practices as Reported by U.S. Technical Reports

\begin{tabular}{lccc|ccc}
\hline \hline & \multicolumn{3}{c|}{ Management } & \multicolumn{3}{c}{ Combined } \\
\cline { 2 - 7 } & Year1 & Year2 & Year3 & Year1 & Year2 & Year3 \\
& $(1)$ & $(2)$ & $(3)$ & $(4)$ & $(5)$ & $(6)$ \\
\hline Managerial Practices & & & & & \\
1) Human Resource Training & & & & & & \\
1a) Training for Leaders & $59 \%$ & $78 \%$ & $90 \%$ & $55 \%$ & $71 \%$ & $87 \%$ \\
1b) Training for Rest of Workers & $73 \%$ & $85 \%$ & $95 \%$ & $75 \%$ & $90 \%$ & $97 \%$ \\
1c) Introduction of Bonuses & $68 \%$ & $81 \%$ & $89 \%$ & $65 \%$ & $77 \%$ & $85 \%$ \\
& & & & & & \\
2) Marketing & & & & & & \\
2a) Market Research and Branding & $65 \%$ & $75 \%$ & $88 \%$ & $70 \%$ & $77 \%$ & $89 \%$ \\
2b) Advertising Campaigns & $79 \%$ & $88 \%$ & $98 \%$ & $73 \%$ & $86 \%$ & $99 \%$ \\
3) Factory Operations & & & & & & \\
3a) Maintenance of Machines & & & & & & \\
3b) Maintenance of Safety & $65 \%$ & $79 \%$ & $87 \%$ & $68 \%$ & $75 \%$ & $86 \%$ \\
4) Production Planning & $71 \%$ & $82 \%$ & $92 \%$ & $70 \%$ & $87 \%$ & $95 \%$ \\
Sales and Orders Management & & & & & & \\
\hline Number of firms & $75 \%$ & $87 \%$ & $95 \%$ & $74 \%$ & $90 \%$ & $97 \%$ \\
\hline \hline
\end{tabular}

Notes. Percentage of firms that adopted U.S. managerial practices 1, 2, and 3 years after the Productivity Program in treatment provinces for firms that chose management transfer (columns 1-6) and firms that chose the combined management and technology transfers (columns 7-12). Data are from the reports compiled by U.S. experts who visited participating plants in the three years after the program. The U.S. managerial practices are based on the Training Within Industry (TWI) method, described in Section 1. 
Table A.24-: Effects of the Productivity Program on Real Wages

\begin{tabular}{lccc}
\hline \hline & \multicolumn{3}{c}{ Log Real Wages (1-3) } \\
\cline { 2 - 4 } & Management & Technology & Combined \\
Year1AfterPP & $(1)$ & $(2)$ & $(3)$ \\
\cline { 2 - 4 } & 0.065 & 0.008 & 0.141 \\
Year5AfterPP & $(0.020)$ & $(0.010)$ & $(0.035)$ \\
& 0.117 & 0.065 & 0.205 \\
Year10AfterPP & $(0.030)$ & $(0.015)$ & $(0.045)$ \\
& 0.198 & 0.089 & 0.253 \\
Year15AfterPP & $(0.045)$ & $(0.064)$ & $(0.055)$ \\
& 0.278 & 0.085 & 0.291 \\
Observations & $(0.059)$ & $(0.061)$ & $(0.068)$ \\
Number of firms & 10,760 & 14,960 & 21,640 \\
\hline Firm FE & 538 & 748 & 1,082 \\
Year FE & Yes & Yes & Yes \\
Sample & Yes & Yes & Yes \\
\hline \hline
\end{tabular}

Notes. Coefficients estimated from equation 1 on 538 firms that chose management transfer (column 1), 748 firms that chose technology transfer (column 2), and 1,082 firms that chose the combined management and technology transfers (column 3) and survived in the 15 years after the Productivity Program. Data are provided at the firm level. The dependent variables are real Wages, converted from 1951 Italian lira to 2010 euro and exchanged at 0.780 euro $=$ USD 1 (columns 1-3). Standard errors are block-bootstrapped at the province level with 200 replications. 
Table A.25-: Heterogeneity Effects: by Access to Credit

\begin{tabular}{|c|c|c|c|c|c|c|c|c|c|}
\hline & \multicolumn{3}{|c|}{ A. Management } & \multicolumn{3}{|c|}{ B. Technology } & \multicolumn{3}{|c|}{ C. Combined } \\
\hline & $\begin{array}{l}\text { Log Sales } \\
(1)\end{array}$ & $\begin{array}{l}\text { Log Employees } \\
(2)\end{array}$ & $\begin{array}{l}\text { Log TFPR } \\
(3)\end{array}$ & $\begin{array}{l}\text { Log Sales } \\
(4)\end{array}$ & $\begin{array}{l}\text { Log Employees } \\
\text { (5) }\end{array}$ & $\begin{array}{l}\text { Log TFPR } \\
(6)\end{array}$ & $\begin{array}{l}\text { Log Sales } \\
(7)\end{array}$ & $\begin{array}{l}\text { Log Employees } \\
(8)\end{array}$ & $\begin{array}{l}\text { Log TFPR } \\
(9)\end{array}$ \\
\hline \multicolumn{10}{|c|}{ I. High Access to Credit } \\
\hline Year1AfterPP & $\begin{array}{l}0.055 \\
(0.011)\end{array}$ & $\begin{array}{l}0.005 \\
(0.004)\end{array}$ & $\begin{array}{l}0.153 \\
(0.029)\end{array}$ & $\begin{array}{l}0.007 \\
(0.008)\end{array}$ & $\begin{array}{l}0.017 \\
(0.013)\end{array}$ & $\begin{array}{l}0.019 \\
(0.017)\end{array}$ & $\begin{array}{l}0.087 \\
(0.015)\end{array}$ & $\begin{array}{l}0.035 \\
(0.006)\end{array}$ & $\begin{array}{l}0.215 \\
(0.039)\end{array}$ \\
\hline Year15AfterPP & $\begin{array}{l}0.369 \\
(0.076)\end{array}$ & $\begin{array}{l}0.326 \\
(0.051)\end{array}$ & $\begin{array}{l}0.445 \\
(0.062)\end{array}$ & $\begin{array}{l}0.067 \\
(0.030)\end{array}$ & $\begin{array}{l}0.069 \\
(0.029)\end{array}$ & $\begin{array}{l}0.117 \\
(0.049)\end{array}$ & $\begin{array}{l}0.404 \\
(0.102)\end{array}$ & $\begin{array}{l}0.520 \\
(0.088)\end{array}$ & $\begin{array}{l}0.657 \\
(0.105)\end{array}$ \\
\hline \multicolumn{10}{|c|}{ II. Low Access to Credit } \\
\hline Year1AfterPP & $\begin{array}{l}0.062 \\
(0.013)\end{array}$ & $\begin{array}{l}0.009 \\
(0.006)\end{array}$ & $\begin{array}{l}0.128 \\
(0.025)\end{array}$ & $\begin{array}{l}0.006 \\
(0.008)\end{array}$ & $\begin{array}{l}0.011 \\
(0.010)\end{array}$ & $\begin{array}{l}0.022 \\
(0.014)\end{array}$ & $\begin{array}{l}0.093 \\
(0.018)\end{array}$ & $\begin{array}{l}0.053 \\
(0.007)\end{array}$ & $\begin{array}{l}0.169 \\
(0.038)\end{array}$ \\
\hline Year15AfterPP & $\begin{array}{l}0.307 \\
(0.081)\end{array}$ & $\begin{array}{l}0.289 \\
(0.049)\end{array}$ & $\begin{array}{l}0.375 \\
(0.051)\end{array}$ & $\begin{array}{l}0.073 \\
(0.035)\end{array}$ & $\begin{array}{l}0.085 \\
(0.039)\end{array}$ & $\begin{array}{l}0.095 \\
(0.042)\end{array}$ & $\begin{array}{l}0.479 \\
(0.105)\end{array}$ & $\begin{array}{l}0.471 \\
(0.099)\end{array}$ & $\begin{array}{l}0.593 \\
(0.114)\end{array}$ \\
\hline Sample & Balanced & Balanced & Balanced & Balanced & Balanced & Balanced & Balanced & Balanced & Balanced \\
\hline Firm FE & Yes & Yes & Yes & Yes & Yes & Yes & Yes & Yes & Yes \\
\hline Year FE & Yes & Yes & Yes & Yes & Yes & Yes & Yes & Yes & Yes \\
\hline$F$-statistic Year1 & 47.9 & 48.9 & 41.7 & 47.5 & 53.6 & 42.7 & 33.3 & 55.8 & 42.3 \\
\hline$F$-statistic Year15 & 35.4 & 47.6 & 32.9 & 30.7 & 41.2 & 53.5 & 45.9 & 38.0 & 51.3 \\
\hline
\end{tabular}

Notes. OLS estimation of equation 1 for 538 firms that chose management transfer (columns 1-3), 748 firms that chose technology transfer (columns 4-6), and 1,082 firms that chose the combined management and technology transfers (columns 7-9) and survived in the 15 years after the Productivity Program, stratifying the sample by access to credit. The dependent variables are logged (deflated) Sales converted from 1951 Italian lira to 2010 euro and exchanged at 0.780 euro = USD 1 (columns 1, 4, and 7); logged Employees, reporting the number of employees per firm (columns 2, 5, and 8); and logged TFPR, estimated using the Ackerberg, Caves and Frazer (2006) method (columns 3, 6, and 9). Data are provided at the firm level. Standard errors are block-bootstrapped at the province level with 200 replications. 
Table A.26-: Multinomial Logit, U.S. Transfers Choice

\begin{tabular}{ll|l|l}
\hline \hline & \multicolumn{3}{c}{ Choice of U.S. Transfer } \\
\cline { 2 - 4 } & Management & Technology & \multicolumn{2}{l}{ Combined } \\
& $(1)$ & $(2)$ & $(3)$ \\
\hline Plants per firm & 0.012 & 0.027 & 0.033 \\
Employees per firm & $(0.006)$ & $(0.009)$ & $(0.011)$ \\
& 0.008 & 0.017 & 0.028 \\
Annual sales (k USD) & $(0.003)$ & $(0.003)$ & $(0.009)$ \\
& 0.015 & 0.013 & 0.022 \\
Productivity (TFPR) & $(0.004)$ & $(0.005)$ & $(0.008)$ \\
Age & 0.021 & 0.016 & 0.025 \\
Export & $(0.006)$ & $(0.004)$ & $(0.008)$ \\
Family-managed & -0.009 & -0.011 & -0.008 \\
Treatment Province & $(0.011)$ & $(0.012)$ & $(0.013)$ \\
& 0.009 & 0.018 & 0.031 \\
Observations & $(0.008)$ & $(0.010)$ & $(0.017)$ \\
Pilot region FE & -0.151 & -0.127 & -0.176 \\
Sector FE & $(0.032)$ & $(0.025)$ & $(0.034)$ \\
\hline \hline
\end{tabular}

Notes. Marginal effects estimated from the multinomial logit model of equation 3, where the choice is either applying for the management transfer, the technology transfer, or the combined management and technology transfers, or not applying, used as baseline. Thirty firms whose applications were rejected are excluded. Plants per firm is the total number of plants per firm; Employees per firm is the number of employees per firm; Annual sales is in 2010 USD, reevaluated from 1951 to 2010 values at 1 lira $=30.884$ euros and exchanged at 0.780 euro $=$ USD 1; Productivity (log TFPR) is the logarithm of firm productivity, estimated using the Ackerberg, Caves and Frazer (2006) method; Export and Familymanaged are indicator variables that equal one if, respectively, a firm exported and was family-managed; treatment province is an indicator for firms located in a treatment province. Data are provided at the firm level. denotes $1 \%$, denotes $5 \%$, and denotes $10 \%$ significance. 
Table A.27-: Spillover Effects on Firms That Did Not Receive U.S. Transfers

\begin{tabular}{|c|c|c|c|c|c|c|c|c|c|c|c|c|}
\hline & \multicolumn{3}{|c|}{ Probability of Shutdown } & \multicolumn{3}{|c|}{ Log Sales } & \multicolumn{3}{|c|}{ Log Employment } & \multicolumn{3}{|c|}{ Log TFPR } \\
\hline & $(1)$ & $(2)$ & $(3)$ & $(4)$ & $(5)$ & $(6)$ & $(7)$ & $(8)$ & $(9)$ & $(10)$ & $(11)$ & $(12)$ \\
\hline Manag.PostPP.Different & $\begin{array}{l}0.002 \\
(0.004)\end{array}$ & $\begin{array}{l}0.002 \\
(0.003)\end{array}$ & $\begin{array}{l}0.002 \\
(0.003)\end{array}$ & $\begin{array}{l}-0.004 \\
(0.006)\end{array}$ & $\begin{array}{l}-0.003 \\
(0.005)\end{array}$ & $\begin{array}{l}-0.002 \\
(0.002)\end{array}$ & $\begin{array}{l}0.006 \\
(0.009)\end{array}$ & $\begin{array}{l}0.005 \\
(0.008)\end{array}$ & $\begin{array}{l}0.003 \\
(0.005)\end{array}$ & $\begin{array}{l}0.009 \\
(0.014)\end{array}$ & $\begin{array}{l}0.007 \\
(0.013)\end{array}$ & $\begin{array}{l}0.007 \\
(0.012)\end{array}$ \\
\hline Tech·PostPP·Different & $\begin{array}{l}0.001 \\
(0.004)\end{array}$ & $\begin{array}{l}-0.001 \\
(0.004)\end{array}$ & $\begin{array}{l}-0.001 \\
(0.004)\end{array}$ & $\begin{array}{l}0.005 \\
(0.007)\end{array}$ & $\begin{array}{l}0.005 \\
(0.007)\end{array}$ & $\begin{array}{l}0.003 \\
(0.004)\end{array}$ & $\begin{array}{c}-0.011 \\
(0.015)\end{array}$ & $\begin{array}{l}-0.010 \\
(0.013)\end{array}$ & $\begin{array}{l}-0.010 \\
(0.010)\end{array}$ & $\begin{array}{l}0.002 \\
(0.005)\end{array}$ & $\begin{array}{l}0.002 \\
(0.004)\end{array}$ & $\begin{array}{l}0.001 \\
(0.03)\end{array}$ \\
\hline Combined·PostPP·Different & $\begin{array}{l}0.004 \\
(0.006)\end{array}$ & $\begin{array}{l}0.003 \\
(0.005)\end{array}$ & $\begin{array}{l}0.003 \\
(0.005)\end{array}$ & $\begin{array}{l}-0.006 \\
(0.008)\end{array}$ & $\begin{array}{l}-0.006 \\
(0.008)\end{array}$ & $\begin{array}{l}-0.004 \\
(0.006)\end{array}$ & $\begin{array}{l}0.014 \\
(0.017)\end{array}$ & $\begin{array}{l}0.012 \\
(0.015)\end{array}$ & $\begin{array}{l}0.009 \\
(0.011)\end{array}$ & $\begin{array}{l}0.008 \\
(0.012)\end{array}$ & $\begin{array}{l}0.007 \\
(0.010)\end{array}$ & $\begin{array}{l}0.007 \\
(0.007)\end{array}$ \\
\hline Manag.PostPP.Samet & $\begin{array}{l}0.012 \\
(0.007)\end{array}$ & $\begin{array}{l}0.012 \\
(0.006)\end{array}$ & $\begin{array}{l}0.002 \\
(0.005)\end{array}$ & $\begin{array}{l}-0.032 \\
(0.019)\end{array}$ & $\begin{array}{l}-0.029 \\
(0.017)\end{array}$ & $\begin{array}{l}0.001 \\
(0.007)\end{array}$ & $\begin{array}{l}-0.007 \\
(0.012)\end{array}$ & $\begin{array}{l}-0.007 \\
(0.011)\end{array}$ & $\begin{array}{l}-0.003 \\
(0.010)\end{array}$ & $\begin{array}{l}-0.017 \\
(0.008)\end{array}$ & $\begin{array}{l}-0.015 \\
(0.007)\end{array}$ & $\begin{array}{l}-0.006 \\
(0.006)\end{array}$ \\
\hline Techn·PostPP·Same & $\begin{array}{l}0.015 \\
(0.009)\end{array}$ & $\begin{array}{l}0.015 \\
(0.009)\end{array}$ & $\begin{array}{l}0.001 \\
(0.007)\end{array}$ & $\begin{array}{l}-0.024 \\
(0.014)\end{array}$ & $\begin{array}{l}-0.021 \\
(0.012)\end{array}$ & $\begin{array}{l}-0.002 \\
(0.006)\end{array}$ & $\begin{array}{c}-0.004 \\
(0.009)\end{array}$ & $\begin{array}{l}-0.004 \\
(0.008)\end{array}$ & $\begin{array}{l}-0.002 \\
(0.004)\end{array}$ & $\begin{array}{c}-0.013 \\
(0.006)\end{array}$ & $\begin{array}{l}-0.012 \\
(0.006)\end{array}$ & $\begin{array}{c}-0.002 \\
(0.005)\end{array}$ \\
\hline Combined·PostPP.Same & $\begin{array}{l}0.014 \\
(0.008)\end{array}$ & $\begin{array}{l}0.014 \\
(0.008)\end{array}$ & $\begin{array}{l}0.002 \\
(0.007)\end{array}$ & $\begin{array}{l}-0.035 \\
(0.020)\end{array}$ & $\begin{array}{l}-0.028 \\
(0.017)\end{array}$ & $\begin{array}{l}-0.011 \\
(0.012)\end{array}$ & $\begin{array}{l}-0.005 \\
(0.008)\end{array}$ & $\begin{array}{l}-0.005 \\
(0.007)\end{array}$ & $\begin{array}{l}-0.002 \\
(0.006)\end{array}$ & $\begin{array}{l}-0.022 \\
(0.010)\end{array}$ & $\begin{array}{l}-0.019 \\
(0.008)\end{array}$ & $\begin{array}{l}-0.009 \\
(0.007)\end{array}$ \\
\hline Observations & 105,400 & 105,400 & 105,400 & 73,780 & 73,780 & 73,780 & 73,780 & 73,780 & 73,780 & 73,780 & 73,780 & 73,780 \\
\hline Radius (km) & 5 & 10 & 20 & 5 & 10 & 20 & 5 & 10 & 20 & 5 & 10 & 20 \\
\hline Panel & Unbal. & Unbal. & Unbal. & Bal. & Bal. & Bal. & Bal. & Bal. & Bal. & Bal. & Bal. & Bal. \\
\hline Firm FE & Yes & Yes & Yes & Yes & Yes & Yes & Yes & Yes & Yes & Yes & Yes & Yes \\
\hline Year FE & Yes & Yes & Yes & Yes & Yes & Yes & Yes & Yes & Yes & Yes & Yes & Yes \\
\hline
\end{tabular}

Notes. Coefficients estimated from equation 4 for 5,270 firms that did not received Productivity Program transfers. In columns 1-3, the samples include all the firms; in columns 4-12, only firms that survived in the 15 years after the Productivity Program. Manag · PostPP · Same, Tech · PostPP · Same, Combined · PostPP - Same are the count of firms that received, respectively, management transfer, technology transfer or the combined management and technology transfers, operating in the same industry and located in the radius of $x$ km from firm $i$, where $x$ is within 5,10 , or $20 \mathrm{~km}$ of a non-participating firm; Manag.PostPP.Different, Tech ·PostPP.Different, and Combined PostPP.Different are the count of firms that received, respectively, management transfer, technology transfer or the combined management and technology transfers, operating in a different industry and located in the radius of $x$ km from firm $i$, where $x$ is within 5, 10, or $20 \mathrm{~km}$ of a non-participating firm. Data are provided at the firm level. The dependent variables are Probability of Shutdown, an indicator for firms that shut down in year $t$; Log Sales, reported in 2010 USD, reevaluated from 1951 to 2010 values at 1 lira $=30.884$ euros and exchanged at 0.780 euro = USD 1; Log Employment, reporting the logged number of employees per firm; and log TFPR, the logarithm of firm productivity, estimated using the Ackerberg, Caves and Frazer (2006) method. Standard errors are block-bootstrapped at the province level with 200 replications. 
Table A.28-: Effects of the Productivity Program on Sales, Employment, and TFPR Controlling for Spillover Effects

\begin{tabular}{|c|c|c|c|c|c|c|c|c|c|}
\hline & \multicolumn{3}{|c|}{ A. Management (1-3) } & \multicolumn{3}{|c|}{ B. Technology $(4-6)$} & \multicolumn{3}{|c|}{ C. Combined (7-9) } \\
\hline & Log Sales & Log Employees & Log TFPR & Log Sales & Log Employees & Log TFPR & Log Sales & Log Employees & Log TFPR \\
\hline & $(1)$ & (2) & (3) & (4) & $(5)$ & (6) & $(7)$ & (8) & (9) \\
\hline \multirow[t]{2}{*}{ Year1AfterPP } & 0.057 & 0.005 & 0.136 & 0.006 & 0.009 & 0.018 & 0.086 & 0.034 & 0.182 \\
\hline & $(0.013)$ & $(0.006)$ & $(0.025)$ & $(0.007)$ & $(0.010)$ & $(0.012)$ & $(0.023)$ & $(0.009)$ & $(0.0232)$ \\
\hline \multirow[t]{2}{*}{ Year5AfterPP } & 0.101 & 0.058 & 0.195 & 0.036 & 0.029 & 0.069 & 0.241 & 0.159 & 0.302 \\
\hline & $(0.026)$ & $(0.020)$ & $(0.038)$ & $(0.013)$ & $(0.014)$ & $(0.019)$ & $(0.033)$ & $(0.032)$ & $(0.0342)$ \\
\hline \multirow[t]{2}{*}{ Year10AfterPP } & 0.186 & 0.193 & 0.265 & 0.063 & 0.067 & 0.096 & 0.279 & 0.346 & 0.455 \\
\hline & $(0.035)$ & $(0.038)$ & $(0.053)$ & $(0.029)$ & $(0.031)$ & $(0.042)$ & $(0.047)$ & $(0.038)$ & $(0.057)$ \\
\hline \multirow[t]{2}{*}{ Year15AfterPP } & 0.313 & 0.278 & 0.468 & 0.060 & 0.069 & 0.095 & 0.428 & 0.473 & 0.597 \\
\hline & $(0.051)$ & $(0.045)$ & $(0.056)$ & $(0.030)$ & $(0.033)$ & $(0.047)$ & $(0.061)$ & $(0.057)$ & $(0.059)$ \\
\hline Observations & 10,760 & 10,760 & 10,760 & 14,960 & 14,960 & 14,960 & 21,640 & 21,640 & 21,640 \\
\hline Number of firms & 538 & 538 & 538 & 748 & 748 & 748 & 1,082 & 1,082 & 1,082 \\
\hline Firm FE & Yes & Yes & Yes & Yes & Yes & Yes & Yes & Yes & Yes \\
\hline Year FE & Yes & Yes & Yes & Yes & Yes & Yes & Yes & Yes & Yes \\
\hline Sample & Balanced & Balanced & Balanced & Balanced & Balanced & Balanced & Balanced & Balanced & Balanced \\
\hline
\end{tabular}

Notes. Coefficients estimated from equation 1 on firms that survived for 15 years after the program, excluding firms within 5 kilometers of a firm that participated in the Productivity Program. Data are provided at the firm level. The dependent variables are logged deflated Sales, converted from 1951 Italian lira to 2010 euro and exchanged at 0.780 euro = USD 1 (columns 1-4); logged Employees, reporting the number of employees per firm (columns 5-8); and logged TFPR, estimated using the Ackerberg, Caves and Frazer (2006) method (columns 9-12). Standard errors are block-bootstrapped at the province level with 200 replications. 


\section{B. Data Collection}

The data collection targeted the population of firms eligible to apply for the Productivity Program in 1951. The process comprised three phases.

Phase 1: Locating the data. Between September and November 2013, I contacted four Italian historical archives: Confindustria Historical Archive (ASC), the Central Archives of the States (ACS), the Historical Archive of Istituto Mobiliare Italiano (ASI-IMI), and the Bank of Italy Historical Archive (ASBI), all located in Rome, Italy. These archives confirmed that they owned the data I needed and granted me access to it.

Phase 2: Collecting the data. Between December 2013 and March 2014, I visited the four archives, proceeding in three steps. First, I used firm registries at ASC to obtain the list of 6,065 firms that were eligible to apply for the Productivity Program in 1951. These firms were manufacturing companies, with 15 to 250 employees, were required to compile a balance sheet, and were located in five Italian regions: Lombardia, Veneto, Toscana, Campania, and Sicilia. Second, for each of them, I photographed the balance sheets and the statement of profits and losses from 1946 to 1973, which are stored at ASC. Finally, I linked these firms with the application records, stored at ACS and ASI-IMI. I was able to take pictures of 60 percent of the application records. For the remaining 40 percent, I was not allowed to take pictures due to archive regulations, so I manually copied them. I also visited the ASBI to obtain institutional data, such as the series of interest rates, GDP, and industries deflators. The ASBI provided this material on a DVD.

Phase 3: Digitizing the data. Between April and December 2014, I digitized the photographic copies with the help of freelancers hired on a popular online marketplace. To test the quality of the freelancers, I prepared a guideline document and tested their ability to transcribe the data into Excel spreadsheets. I hired only freelancers who made zero mistakes in this phase. To ensure quality of the data, I had two freelancers digitizing the same data. This tactic sped up the search for potential mistakes. In particular, I checked all the data by comparing the work of the two freelancers. For each difference I found, I manually checked the original document and fixed the mistake. In addition, I randomly checked 10 percent of the digitized data for which there were no differences. Finally, I manually matched the eligible firms with the application records, using firm name, headquarters address, and municipality as identifiers. 


\section{Description of Primary Sources}

The main source of data for this paper is firm balance sheets. According to 1942 Italian civil code, firms with at least $2010 \$ 150,000$ in annual revenues had to compile a balance sheet. This was one of the eligibility criteria for firms to participate in the Productivity Program. Italian balance sheets are composed of three parts: the stato patrimoniale, the conto economico, and the nota integrativa. The stato patrimoniale is the statement of assets and liabilities. The conto economico is the statement of profits and losses. The nota integrativa is a note attached to the balance sheets that provides additional data that, given their qualitative or extra-accounting nature, are not reported in the other documents. Appendix Table C.1 contains a list and definition of all the variables used in the paper and their sources. 
Table C.1-: List and Definition of Variables and Their Sources

\begin{tabular}{|c|c|c|}
\hline Variable & Definition & Source \\
\hline Sales & Operating Revenues & Conto Economico \\
\hline Employment & Number of Employees & Nota Integrativa \\
\hline Productivity (TFPR) & Total Factor Productivity Revenue & Author's Calculation (see Appendix E.1) \\
\hline Revenues & Gross Income & Conto Economico \\
\hline Value Added & Difference between firm gross income and intermediate inputs & Author's Calculation (see Appendix E.1) \\
\hline Profits & Difference between value added and taxes & Author's Calculation \\
\hline Intermediate Inputs & Sum of costs of raw materials & Conto Economico \\
\hline Capital & Firm capital stock & Author's Calculation (see Appendix E.1) \\
\hline Import & Values of imported goods & Conto Economico \\
\hline Export & Values of exported goods & Conto Economico \\
\hline Wages & Total wages paid to workers & Conto Economico \\
\hline Investments & Difference between fixed gross asset at time $t$ and time $t-1$ & Author's Calculation (see Appendix E.1) \\
\hline Loans & Firm loans with banks & Stato Patrimoniale \\
\hline Fixed Gross Asset & Value of land, buildings, and machines owned by the firm & Stato Patrimoniale \\
\hline Managers & Numbers and names of managers & Nota Integrativa \\
\hline Professionally-managed firm & Firm with no family representative or kin formally involved in its governance & Nota Integrativa \\
\hline Return-on-Assets (ROA) & Ratio between profits and capital & Author's Calculation \\
\hline
\end{tabular}




\section{Robustness Checks}

\section{A. Alternative Specifications}

To be consistent with the event study presented in Section 4.1 and to exploit the variation at the province level, I also compare the outcomes of firms located in treatment provinces with those of firms located in comparison provinces in the same pilot region and that applied for the same transfer in 1951 via the following equation:

(D.1) outcome $_{\text {isprt }}=\alpha+\beta$ Treat $_{p}+\sum_{\tau=-5}^{15} \delta_{\tau}\left(\right.$ Treat $\left._{p} \cdot \operatorname{PostPP}_{\tau}\right)+\lambda_{r}+\zeta_{s}+\nu_{t}+\epsilon_{\text {isprt }}$

where the dependent variable, outcome isprt $_{\text {, }}$, is one of the key performance metrics of logged (deflated) sales, number of employees, and TFPR of firm $i$ operating in industry $s$, located in province $p$ in region $r$ at time $t . \alpha$ is a constant term; Treat $_{p}$ is an indicator that equals one if firm $i$ is located in a treatment province; $\mathrm{PostPP}_{\tau}$ is an indicator for each year $t$, after firm $i$ received the Productivity Program assistance, from 5 years before to 15 years after the program. Pilot region fixed effects $\lambda_{r}$ control for variation in outcomes across regions that are constant over time; industry fixed effects $\zeta_{s}$ control for variation in outcomes across manufacturing industries; time fixed effects $\nu_{t}$ control for variation in outcomes over time that is common across all Italian regions. $\epsilon_{i s p r t}$ is the error term. Each $\delta_{\tau}$ coefficient captures the effects of the Productivity Program $\tau$ years after its implementation.

Since comparison firms never got treated, I need to assign them a "treatment" year. The Productivity Program was implemented between 1952 and 1958. Firms in treatment provinces were ranked based on their application's submission date, and they received U.S. transfers in the order in which their applications were received. Since I can also observe the application date for firms in comparison provinces, I assume that these firms would have received U.S. assistance in the same year as the firms in treatment provinces that applied at the same time. For instance, if firms in treatment provinces that applied between February 1 and February 20, 1951, received the U.S. assistance in 1953, I assume that firms in comparison provinces that submitted an application between February 1 and February 20, 1951, would have received the U.S. assistance in 1953 as well. I show that the distribution of application dates and the resulting distribution of treatment years for treatment and comparison provinces is balanced (Appendix Figures D.1 and D.2).

The estimates are consistent with the main results presented in Section 4 . The management transfer and the combined management and technology transfers were large and continued to grow for the 15 years after the program, while the effects of technology transfer reached a plateau after 10 years (Appendix Table 
D.1). In terms of magnitude, they are larger than the main results in the paper because I am not using firm fixed effects. The estimation with firm fixed effects are comparable with the main results.

In all the specifications presented so far, to follow each firm for the same number of years before and after the Productivity Program, I restrict the years of data from 5 years before to 15 years after the U.S. intervention. However, I collected data from 1946 to 1973 for all eligible firms, unless they exit the market. I therefore run two additional specifications: a specification in which I keep years from 5 before to 15 after the Productivity Program for treated firms and all the data I have for comparison firms, and a specification in which I keep all the data I have for both treated and comparison firms.

The results, prensented in Appendix Table D.2, are larger than those on the balanced sample I use in the main specification of the paper (Table 3, columns 1, 5, and 9, Panels A-C). This is consistent with the idea that the balanced sample, which conditions on survival, is the most conservative way to estimate the treatment effects. In fact, comparison firms that survived even without participating in the Productivity Program were likely to perform better than comparison firms that failed. The estimates using years from 5 before to 15 after the Productivity Program for treated firms and all the data for comparison firms are substantially equivalent to the estimates on the unbalanced sample (Appendix Table D.2, columns 1, 3, and 5, Panels A-C vs Table 3, columns 3, 7, and 11, Panels A-C). In fact, conceptually, using more data for the comparison firms, but not for the treated firms, should only affect the estimation of year fixed effects, and not of the treatment coefficients, that are estimated for the treated firms only. The estimates using all years of data for both the treated and the comparison firms allows me to estimate additional coefficients up to 21 years after the Productivity Program. The results, comparable to the estimates on the unbalanced sample, confirm that the effects of management and the combined management and technology transfers are persistent over time, and that the effects of the technology transfer flatten out after 10 years (Appendix Table D.2, columns 2, 4, and 6, Panels A-C). I conclude that my results are not driven by restricting the sample from 5 years before to 15 years after the U.S. intervention and are robust to the inclusion of the additional years.

\section{B. Selection of Treatment Provinces}

A possible threat to the identification strategy of this paper is that the selection of firms participating in the program was not random. If firms that eventually took part in the Productivity Program had been randomly selected, I simply could have compared treated and comparison firms in the post-Productivity Program period. The estimates I obtained excluding the pre-program period are essentially the same as the difference-in-differences coefficients, confirming that the province selection after the budget cut was plausibly exogenous (Appendix Table D.3). 


\section{Robustness to Reporting Effects}

Sales, employment, and TFPR come from firm balance sheets. Given the selfreported nature of the balance sheet data, concern arises about both misreporting and changes in reporting behavior caused by the Productivity Program. These effects are unlikely to be a major factor in this research, for a number of reasons. First, from technical reports compiled by the U.S. experts visiting Italian plants, it seems that firm performance improved due to changes in management practices and production technology rather than changes in accounting methods. Second, survivorship and employment that are not subject to reporting errors follow the same dynamics as sales and TFPR. Third, the Productivity Program did not organize specific sessions for reporting and accounting, and I do not observe changes in the balance-sheet structure of receiving firms after the program.

Another concern is the Hawthorne effects. Simply being part of the Productivity Program may have improved firm performance. For instance, study-trip participants or employees could have been more motivated or have worked harder during this time. Although the data do not allow me to rule out this possibility, Hawthorne-type effects should dissipate in the long run and therefore cannot explain why the impact of the Productivity Program persisted over time.

\section{Price Effects}

The improved performance of firms receiving U.S. transfers may have enabled them to increase their market power, and therefore charge higher prices.

I derive a firm-level markups estimation by using the method pioneered by De Loecker and Warzynski (2012). ${ }^{1}$ This approach computes markup without relying on market-level demand information; but it requires only standard firm-level data. The main assumptions are that at least one input is variable (therefore, not subject to adjustment costs) and that firms minimize costs. The intuition is as follows: Under cost minimization, the output elasticity of a variable factor of production is equal to its expenditure share in total revenue only when price equals marginal cost of production. Under any form of imperfect competition, however, the relevant markup drives a wedge between the input's revenue share and its output elasticity. Therefore, the markup is calculated via $\mu_{i t}=\underbrace{\left(\frac{\partial Q_{i t}(\cdot)}{\partial X_{i t}} \cdot \frac{X_{i t}}{Q_{i t}}\right)}_{\text {output elasticity }} / \underbrace{\left(\frac{p_{i t}^{X} X_{i t}}{p_{i t}^{Q} Q_{i t}}\right)}_{\text {revenue share }}$, where $\mu_{i t}$ is the markup of firm $i$ at time $t$, $Q_{i t}$ is firm output, $X_{i t}$ is the variable input, $p_{i t}^{X} X$ is the expenditure on input $X$, and $p_{i t}^{Q} Q$ is total revenue. I estimate the output elasticity from the production function, using the Ackerberg, Caves and Frazer (2006) method, where the vari-

\footnotetext{
${ }^{1}$ To compute markups directly, I would need to observe output, which is not reported in balance sheets.
} 
able input is labor, ${ }^{2}$ and the production output is proxied by deflated revenues. ${ }^{3}$ I calculate the revenue share of labor from balance sheets.

I estimate equation 1 using markup as the dependent variable. In the first five years after the U.S. intervention, the markup remains almost unchanged, then it increases over time, but at a very slow pace (Appendix Table D.4). I then reestimate equation 1 controlling for markup variation over time. One year after the Productivity Program, the estimates are very close in magnitude to those from equation 1 for all the transfers and all the outcomes (Appendix Table D.5). Between five and fifteen years after the U.S. intervention, the estimates that control for markup are smaller than the estimates from equation 1 . The pattern over time, however, is fully consistent. Therefore, the change in market power of firms that participated in the Productivity Program is correlated with its long-run effects, but cannot entirely explain its pattern over time, suggesting that there were productivity improvements.

\section{E. Inverse Probability of Treatment Weighting (IPTW)}

Firms that participated in the Productivity Program could decide the transfer they wanted to receive. As a result, it is likely that businesses that chose the management transfer were different from companies that chose the technology or the combined management and technology transfers.

I employ the inverse probability of treatment weighting (IPTW) using the propensity score to create a synthetic sample in which the distribution of firm covariates is independent from the U.S. intervention they chose. Specifically, I first estimate the propensity score $\hat{p}_{i j}$, the predicted probability of choosing a given U.S. intervention, ${ }^{4}$ as a function of firm covariates (size, assets, sales, productivity, exports, and family ownership in 1951) using a multinomial logit. Second, I weight each observation by the inverse of its propensity score. ${ }^{5}$ Third, I estimate equation 2.

The difference in the weighted means using the inverse propensity score weights among the different interventions and the treatment and comparison groups are

\footnotetext{
${ }^{2}$ I chose labor as the variable input because during the 1950 s and the 1960 s in Italy small and mediumsize firms had little exposure to the unions and were fairly flexible in their management of employees (Zamagni, 1997).

${ }^{3}$ I use deflated revenues, instead of physical output, in computing the output elasticity, which is potentially subject to the omitted price variable bias discussed in Klette and Griliches (1996). This, if anything, might downward-bias the estimates of the markup. However, under Cobb-Douglas technology, the output elasticity reduces to a constant; therefore, the bias induced by unobserved prices impacts only the level of markup but not how it changes over time, which is the outcome of interest in this context. Additional details can be found in De Loecker and Warzynski (2012).

${ }^{4}$ The predicted probability of choosing a given U.S. intervention is $\hat{p}_{i, j=M A N}$ for firms that chose the management transfer; $\hat{p}_{i, j=T E C}$ for firms that chose the technology transfer; and $\left(1-\hat{p}_{i, j=M A N}-\right.$ $\hat{p}_{i, j=T E C}$ ) for firms that chose the combined management and technology transfers.

$5 \frac{1}{\hat{p}_{i, M A N}}$ for firms that chose the management transfer; $\frac{1}{\hat{p}_{i, T E C}}$ for firms that chose the technology transfer; and $\frac{1}{\left(1-\hat{p}_{i, M A N}-\hat{p}_{i, T E C}\right)}$ for firms that chose the combined management and technology transfers.
} 
very balanced (Appendix Table D.6). None of the covariates are statistically significantly different at 1 percent. 


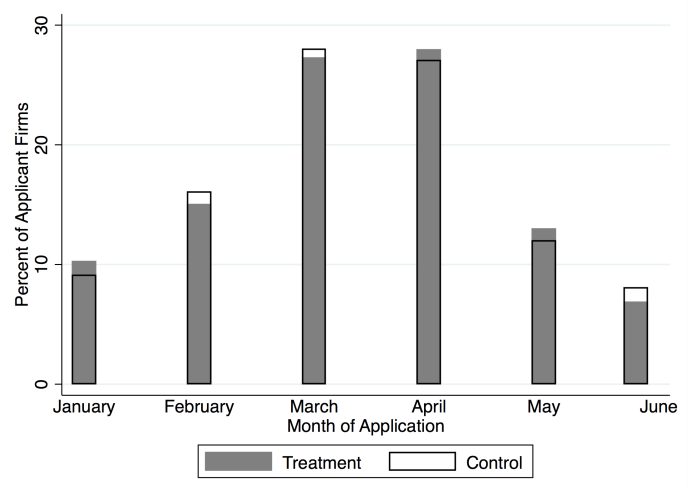

Panel A: Management

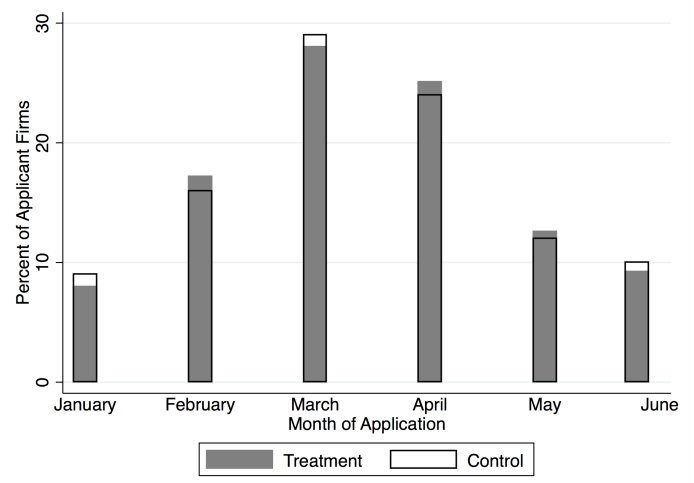

Panel B: Technology

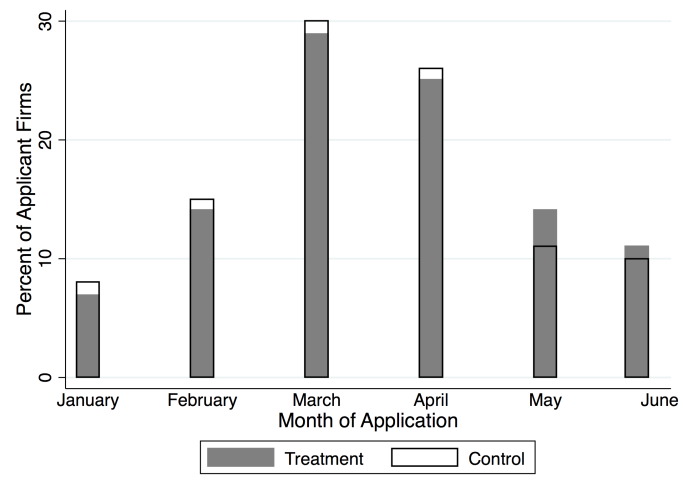

Panel C: Combined

Notes. Distribution of application months for 731 firms that applied for management transfer (Panel A), 1,052 firms that applied for technology transfer (Panel B), and 1,468 firms that applied for the combined management and technology transfers (Panel C). In each panel, the gray bar is for the treated firms, the white bar for the comparison firms.

Figure D.1. : Distribution of Application Months between Treated and Comparison Firms 


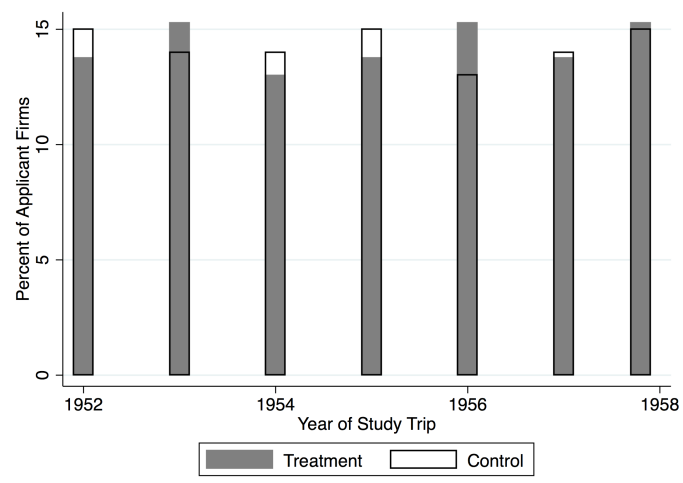

Panel A: Management

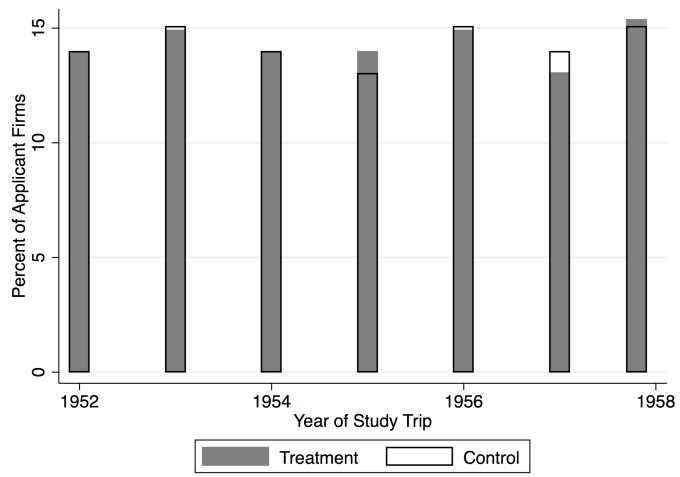

Panel B: Technology

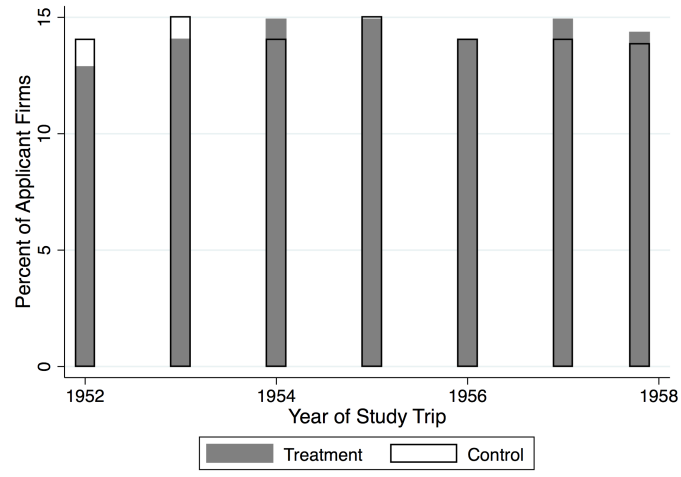

Panel C: Combined

Notes. Distribution of years in which firms participated in the Productivity Program for 731 firms that applied for management transfer (Panel A), 1,052 firms that applied for technology transfer (Panel B), and 1,468 firms that applied for the combined management and technology transfers (Panel C). In each panel, the gray bar is for the treated firms, the white bar for the comparison firms.

Figure D.2. : Distribution of Treatment Years between Treated and Comparison Firms 
Table D.1-: Effects of the Productivity Program on Sales, Employment, and TFPR

\begin{tabular}{|c|c|c|c|c|c|c|c|c|c|}
\hline & \multicolumn{3}{|c|}{ Log Sales $(1-3)$} & \multicolumn{3}{|c|}{ Log Employees (4-6) } & \multicolumn{3}{|c|}{ Log TFPR (7-9) } \\
\hline & $(1)$ & $(2)$ & $(3)$ & $(4)$ & $(5)$ & $(6)$ & $(7)$ & $(8)$ & $(9)$ \\
\hline \multicolumn{10}{|l|}{ A. Management } \\
\hline \multirow[t]{2}{*}{ Year1AfterPP } & 0.070 & 0.063 & 0.073 & 0.011 & 0.008 & 0.012 & 0.153 & 0.146 & 0.159 \\
\hline & $(0.014)$ & $(0.011)$ & $(0.010)$ & $(0.015)$ & $(0.011)$ & $(0.013)$ & $(0.031)$ & $(0.027)$ & $(0.027)$ \\
\hline \multirow[t]{2}{*}{ Year5AfterPP } & 0.125 & 0.119 & 0.142 & 0.069 & 0.067 & 0.076 & 0.221 & 0.215 & 0.234 \\
\hline & $(0.025)$ & $(0.022)$ & $(0.027)$ & $(0.019)$ & $(0.016)$ & $(0.023)$ & $(0.037)$ & $(0.032)$ & $(0.039)$ \\
\hline \multirow[t]{2}{*}{ Year10AfterPP } & 0.208 & 0.205 & 0.235 & 0.219 & 0.209 & 0.257 & 0.312 & 0.303 & 0.341 \\
\hline & $(0.031)$ & $(0.029)$ & $(0.045)$ & $(0.046)$ & $(0.038)$ & $(0.051)$ & $(0.051)$ & $(0.049)$ & $(0.055)$ \\
\hline \multirow[t]{2}{*}{ Year15AfterPP } & 0.354 & 0.344 & 0.406 & 0.326 & 0.312 & 0.384 & 0.421 & 0.414 & 0.473 \\
\hline & $(0.049)$ & $(0.043)$ & $(0.061)$ & $(0.054)$ & $(0.047)$ & $(0.073)$ & $(0.065)$ & $(0.044)$ & $(0.079)$ \\
\hline Observations & 10,760 & 10,760 & 13,902 & 10,760 & 10,760 & 13,902 & 10,760 & 10,760 & 13,902 \\
\hline Number of firms & 538 & 538 & 731 & 538 & 538 & 731 & 538 & 538 & 731 \\
\hline \multicolumn{10}{|l|}{ B. Technology } \\
\hline \multirow[t]{2}{*}{ Year1AfterPP } & 0.013 & 0.009 & 0.015 & 0.018 & 0.013 & 0.021 & 0.028 & 0.023 & 0.032 \\
\hline & $(0.019)$ & $(0.014)$ & $(0.016)$ & $(0.022)$ & $(0.017)$ & $(0.025)$ & $(0.038)$ & $(0.027)$ & $(0.033)$ \\
\hline \multirow[t]{2}{*}{ Year5AfterPP } & 0.051 & 0.047 & 0.058 & 0.041 & 0.037 & 0.047 & 0.083 & 0.079 & 0.091 \\
\hline & $(0.017)$ & $(0.014)$ & $(0.015)$ & $(0.018)$ & $(0.016)$ & $(0.023)$ & $(0.022)$ & $(0.019)$ & $(0.025)$ \\
\hline \multirow[t]{2}{*}{ Year10AfterPP } & 0.081 & 0.075 & 0.094 & 0.084 & 0.082 & 0.095 & 0.111 & 0.115 & 0.121 \\
\hline & $(0.030)$ & $(0.027)$ & $(0.034)$ & $(0.039)$ & $(0.036)$ & $(0.042)$ & $(0.037)$ & $(0.030)$ & $(0.047)$ \\
\hline \multirow[t]{2}{*}{ Year15AfterPP } & 0.079 & 0.076 & 0.084 & 0.085 & 0.080 & 0.095 & 0.108 & 0.102 & 0.122 \\
\hline & $(0.039)$ & $(0.033)$ & $(0.042)$ & $(0.043)$ & $(0.040)$ & $(0.044)$ & $(0.038)$ & $(0.031)$ & $(0.045)$ \\
\hline Observations & 14,960 & 14,960 & 20,213 & 14,960 & 14,960 & 20,213 & 14,960 & 14,960 & 20,213 \\
\hline Number of firms & 748 & 748 & 1,053 & 748 & 748 & 1,053 & 748 & 748 & 1,053 \\
\hline Sample & Balanced & Balanced & Unbalanced & Balanced & Balanced & Unbalanced & Balanced & Balanced & Unbalanced \\
\hline Pilot region $\mathrm{FE}$ & Yes & No & Yes & Yes & No & Yes & Yes & No & Yes \\
\hline Time FE & Yes & Yes & Yes & Yes & Yes & Yes & Yes & Yes & Yes \\
\hline Industry FE & Yes & No & Yes & Yes & No & Yes & Yes & No & Yes \\
\hline Firm FE & No & Yes & No & No & Yes & No & No & Yes & No \\
\hline
\end{tabular}


Table D.1-: Continued

\begin{tabular}{|c|c|c|c|c|c|c|c|c|c|}
\hline & \multicolumn{3}{|c|}{ Log Sales (1-3) } & \multicolumn{3}{|c|}{ Log Employees (4-6) } & \multicolumn{3}{|c|}{ Log TFPR (7-9) } \\
\hline & (1) & $(2)$ & (3) & (4) & $(5)$ & (6) & $(7)$ & $(8)$ & (9) \\
\hline \multicolumn{10}{|l|}{ C. Combined } \\
\hline \multirow[t]{2}{*}{ Year1AfterPP } & 0.092 & 0.087 & 0.094 & 0.049 & 0.044 & 0.051 & 0.208 & 0.199 & 0.212 \\
\hline & $(0.019)$ & $(0.015)$ & $(0.017)$ & $(0.017)$ & $(0.015)$ & $(0.014)$ & $(0.044)$ & $(0.041)$ & $(0.053)$ \\
\hline \multirow[t]{2}{*}{ Year5AfterPP } & 0.252 & 0.244 & 0.279 & 0.185 & 0.181 & 0.197 & 0.351 & 0.347 & 0.358 \\
\hline & $(0.021)$ & $(0.023)$ & $(0.025)$ & $(0.043)$ & $(0.038)$ & $(0.059)$ & $(0.044)$ & $(0.040)$ & $(0.049)$ \\
\hline \multirow[t]{2}{*}{ Year10AfterPP } & 0.310 & 0.290 & 0.369 & 0.389 & 0.374 & 0.429 & 0.505 & 0.500 & 0.533 \\
\hline & $(0.039)$ & $(0.034)$ & $(0.034)$ & $(0.056)$ & $(0.054)$ & $(0.068)$ & $(0.067)$ & $(0.063)$ & $(0.071)$ \\
\hline \multirow[t]{2}{*}{ Year15AfterPP } & 0.470 & 0.459 & 0.602 & 0.530 & 0.513 & 0.591 & 0.653 & 0.645 & 0.725 \\
\hline & $(0.058)$ & $(0.062)$ & $(0.059)$ & $(0.081)$ & $(0.075)$ & $(0.085)$ & $(0.055)$ & $(0.049)$ & $(0.085)$ \\
\hline Observations & 21,640 & 21,640 & 27,870 & 21,640 & 21,640 & 27,870 & 21,640 & 21,640 & 27,870 \\
\hline Number of firms & 1,082 & 1,082 & 1,468 & 1,082 & 1,082 & 1,468 & 1,082 & 1,082 & 1,468 \\
\hline Sample & Balanced & Balanced & Unbalanced & Balanced & Balanced & Unbalanced & Balanced & Balanced & Unbalanced \\
\hline Pilot region FE & Yes & No & Yes & Yes & No & Yes & Yes & No & Yes \\
\hline Time FE & Yes & Yes & Yes & Yes & Yes & Yes & Yes & Yes & Yes \\
\hline Industry FE & Yes & No & Yes & Yes & No & Yes & Yes & No & Yes \\
\hline Firm FE & No & Yes & No & No & Yes & No & No & Yes & No \\
\hline
\end{tabular}

Notes. Coefficients estimated from equation D.1 for firms that applied for management transfer (Panel A), firms that applied for technology transfer (Panel B) and firms that applied for the combined management and technology transfers (Panel C). In columns 1-2, 4-5, and 7-8, the samples include only firms that survived in the 15 years after the Productivity Program; in columns 3, 7, and 11, equation D.1 is estimated on the unbalanced panel (i.e., including firms that exited the market over time) from 5 years before to 15 years after the Productivity Program. If a firm exits the market in year $t$, missing values are imputed for the dependent variables starting on year $t+1$. Data are provided at the firm level. The dependent variables are logged deflated Sales, converted from 1951 Italian lira to 2010 euro and exchanged at 0.780 euro = USD 1 (columns 1-3); logged Employees, reporting the number of employees per firm (columns 4-6); and logged TFPR, estimated using the Ackerberg, Caves and Frazer (2006) method (columns 7-9). Standard errors are block-bootstrapped at the province level with 200 replications. 
Table D.2-: Effects of the Productivity Program on Sales, Employment, and TFPR

\begin{tabular}{|c|c|c|c|c|c|c|}
\hline & \multicolumn{2}{|c|}{ Log Sales (1-2) } & \multicolumn{2}{|c|}{ Log Employees (3-4) } & \multicolumn{2}{|c|}{ Log TFPR (5-6) } \\
\hline & (1) & $(2)$ & $(3)$ & $(4)$ & $(5)$ & $(6)$ \\
\hline \multicolumn{7}{|l|}{ A. Management } \\
\hline \multirow[t]{2}{*}{ Year1AfterPP } & 0.065 & 0.069 & 0.015 & 0.018 & 0.115 & 0.121 \\
\hline & $(0.024)$ & $(0.026)$ & $(0.014)$ & $(0.019)$ & $(0.030)$ & $(0.033)$ \\
\hline \multirow[t]{2}{*}{ Year5AfterPP } & 0.125 & 0.138 & 0.083 & 0.091 & 0.202 & 0.212 \\
\hline & $(0.036)$ & $(0.041)$ & $(0.028)$ & $(0.031)$ & $(0.051)$ & $(0.054)$ \\
\hline \multirow[t]{2}{*}{ Year10AfterPP } & 0.223 & 0.239 & 0.258 & 0.268 & 0.306 & 0.318 \\
\hline & $(0.053)$ & $(0.059)$ & $(0.048)$ & $(0.055)$ & $(0.065)$ & $(0.071)$ \\
\hline \multirow[t]{2}{*}{ Year15AfterPP } & 0.383 & 0.402 & 0.354 & 0.366 & 0.387 & 0.398 \\
\hline & $(0.070)$ & $(0.079)$ & $(0.061)$ & $(0.074)$ & $(0.085)$ & $(0.094)$ \\
\hline \multirow[t]{2}{*}{ Year21AfterPP } & & 0.433 & & 0.391 & & 0.443 \\
\hline & & $(0.088)$ & & $(0.092)$ & & $(0.109)$ \\
\hline Observations & 15,678 & 17,530 & 15,678 & 17,530 & 15,678 & 17,530 \\
\hline Number of firms & 731 & 731 & 731 & 731 & 731 & 731 \\
\hline \multicolumn{7}{|l|}{ B. Technology } \\
\hline \multirow[t]{2}{*}{ Year1AfterPP } & 0.010 & 0.011 & 0.021 & 0.024 & 0.027 & 0.031 \\
\hline & $(0.012)$ & $(0.015)$ & $(0.015)$ & $(0.016)$ & $(0.020)$ & $(0.023)$ \\
\hline \multirow[t]{2}{*}{ Year5AfterPP } & 0.052 & 0.062 & 0.055 & 0.061 & 0.085 & 0.092 \\
\hline & $(0.018)$ & $(0.024)$ & $(0.020)$ & $(0.023)$ & $(0.028)$ & $(0.030)$ \\
\hline \multirow[t]{2}{*}{ Year10AfterPP } & 0.091 & 0.098 & 0.091 & 0.102 & 0.128 & 0.136 \\
\hline & $(0.039)$ & $(0.045)$ & $(0.046)$ & $(0.050)$ & $(0.055)$ & $(0.064)$ \\
\hline \multirow[t]{2}{*}{ Year15AfterPP } & 0.089 & 0.093 & 0.090 & 0.099 & 0.129 & 0.130 \\
\hline & $(0.051)$ & $(0.055)$ & $(0.044)$ & $(0.047)$ & $(0.058)$ & $(0.061)$ \\
\hline \multirow[t]{2}{*}{ Year21AfterPP } & & 0.090 & & 0.095 & & 0.128 \\
\hline & & $(0.062)$ & & $(0.054)$ & & $(0.069)$ \\
\hline Observations & 23,782 & 26,946 & 23,782 & 26,946 & 23,782 & 26,946 \\
\hline Number of firms & 1,053 & 1,053 & 1,053 & 1,053 & 1,053 & 1,053 \\
\hline \multicolumn{7}{|l|}{ C. Combined } \\
\hline \multirow[t]{2}{*}{ Year1AfterPP } & 0.099 & 0.105 & 0.046 & 0.056 & 0.205 & 0.219 \\
\hline & $(0.030)$ & $(0.033)$ & $(0.015)$ & $(0.020)$ & $(0.044)$ & $(0.047)$ \\
\hline \multirow[t]{2}{*}{ Year5AfterPP } & 0.278 & 0.286 & 0.203 & 0.219 & 0.358 & 0.381 \\
\hline & $(0.055)$ & $(0.061)$ & $(0.055)$ & $(0.060)$ & $(0.058)$ & $(0.065)$ \\
\hline \multirow[t]{2}{*}{ Year10AfterPP } & 0.372 & 0.391 & 0.402 & 0.420 & 0.512 & 0.528 \\
\hline & $(0.067)$ & $(0.071)$ & $(0.066)$ & $(0.075)$ & $(0.071)$ & $(0.080)$ \\
\hline \multirow[t]{2}{*}{ Year15AfterPP } & 0.504 & 0.529 & 0.546 & 0.559 & 0.674 & 0.685 \\
\hline & $(0.111)$ & $(0.121)$ & $(0.071)$ & $(0.078)$ & $(0.094)$ & $(0.101)$ \\
\hline \multirow[t]{2}{*}{ Year21AfterPP } & & 0.561 & & 0.589 & & 0.710 \\
\hline & & $(0.133)$ & & $(0.090)$ & & $(0.109)$ \\
\hline Observations & 34,588 & 37,010 & 34,588 & 37,010 & 34,588 & 37,010 \\
\hline Number of firms & 1,468 & 1,468 & 1,468 & 1,468 & 1,468 & 1,468 \\
\hline
\end{tabular}

Notes. Columns 1, 3, and 5 report the coefficients from equation 1 estimated by including data from 5 years before to 15 years after for treated firms and all the years of data available for comparison firms. Columns 2, 4, and 6 report the coefficients from equation 1 estimated by including all the available years of data for both treated and comparison firms. The dependent variables are logged deflated Sales, converted from 1951 Italian lira to 2010 euro and exchanged at 0.780 euro=USD 1 (columns 1-2); logged Employment, reporting the number of employees per firm (columns 3-4); and logged TFPR, estimated using the Ackerberg, Caves and Frazer (2006) method (columns 5-6). Standard errors are block-bootstrapped at the province level with 200 replications. 
Table D.3-: Effects of the Productivity Program on Sales, Employment, and TFPR (Using Only Post Productivity Program Data)

\begin{tabular}{|c|c|c|c|c|c|c|c|c|c|}
\hline & \multicolumn{3}{|c|}{ A. Management } & \multicolumn{3}{|c|}{ B. Technology } & \multicolumn{3}{|c|}{ C. Combined } \\
\hline & $\begin{array}{l}\text { Log sales } \\
(1)\end{array}$ & $\begin{array}{l}\text { Log employees } \\
(2)\end{array}$ & $\begin{array}{l}\text { Log TFPR } \\
(3)\end{array}$ & $\begin{array}{l}\text { Log sales } \\
(4)\end{array}$ & $\begin{array}{l}\text { Log employees } \\
(5)\end{array}$ & $\begin{array}{l}\text { Log TFPR } \\
(6)\end{array}$ & $\begin{array}{l}\text { Log sales } \\
(7)\end{array}$ & $\begin{array}{l}\text { Log employees } \\
(8)\end{array}$ & $\begin{array}{l}\text { Log TFPR } \\
(9)\end{array}$ \\
\hline \multicolumn{10}{|l|}{ A. Management } \\
\hline \multirow{2}{*}{ Year1AfterPP } & 0.062 & 0.010 & 0.135 & 0.011 & 0.015 & 0.025 & 0.087 & 0.035 & 0.203 \\
\hline & $(0.011)$ & $(0.014)$ & $(0.030)$ & $(0.017)$ & $(0.019)$ & $(0.034)$ & $(0.021)$ & $(0.015)$ & $(0.041)$ \\
\hline \multirow[t]{2}{*}{ Year5AfterPP } & 0.113 & 0.065 & 0.200 & 0.049 & 0.039 & 0.079 & 0.238 & 0.170 & 0.328 \\
\hline & $(0.020)$ & $(0.021)$ & $(0.041)$ & $(0.015)$ & $(0.017)$ & $(0.020)$ & $(0.024)$ & $(0.040)$ & $(0.039)$ \\
\hline \multirow[t]{2}{*}{ Year10AfterPP } & 0.188 & 0.202 & 0.278 & 0.077 & 0.080 & 0.117 & 0.287 & 0.333 & 0.449 \\
\hline & $(0.033)$ & $(0.049)$ & $(0.046)$ & $(0.027)$ & $(0.040)$ & $(0.032)$ & $(0.043)$ & $(0.052)$ & $(0.062)$ \\
\hline \multirow[t]{2}{*}{ Year15AfterPP } & 0.328 & 0.308 & 0.385 & 0.073 & 0.081 & 0.109 & 0.431 & 0.525 & 0.607 \\
\hline & $(0.051)$ & $(0.057)$ & $(0.060)$ & $(0.037)$ & $(0.041)$ & $(0.042)$ & $(0.062)$ & $(0.077)$ & $(0.049)$ \\
\hline Observations & 8,070 & 8,070 & 8,070 & 11,220 & 11,220 & 11,220 & 16,230 & 16,230 & 16,230 \\
\hline Number of firms & 538 & 538 & 538 & 748 & 748 & 748 & 1,082 & 1,082 & 1,082 \\
\hline Sample & Balanced & Balanced & Balanced & Balanced & Balanced & Balanced & Balanced & Balanced & Balanced \\
\hline Firm FE & Yes & Yes & Yes & Yes & Yes & Yes & Yes & Yes & Yes \\
\hline Year FE & Yes & Yes & Yes & Yes & Yes & Yes & Yes & Yes & Yes \\
\hline
\end{tabular}

Notes. Coefficients estimated from equation 1 using only post Productivity Program years. Data are provided at the firm level. The dependent variables are logged deflated Sales, converted from 1951 Italian lira to 2010 euro and exchanged at 0.780 euro=USD 1 (columns 1-4); logged Employment, reporting the number of employees per firm (columns 5-8); and logged TFPR, estimated using the Ackerberg, Caves and Frazer (2006) method (columns 9-12). Standard errors are block-bootstrapped at the province level with 200 replications. 
Table D.4-: Effects of the Productivity Program on Markups

\begin{tabular}{ll|l|l}
\hline \hline & A.Management & B. Technology & C. Combined \\
\cline { 2 - 4 } & $\begin{array}{l}\text { Log Markups } \\
(1)\end{array}$ & $\begin{array}{l}\text { Log Markups } \\
(2)\end{array}$ & $\begin{array}{l}\text { Log Markups } \\
(3)\end{array}$ \\
\hline Year1AfterPP & 0.007 & 0.002 & 0.005 \\
& $(0.005)$ & $(0.002)$ & $(0.004)$ \\
Year5AfterPP & 0.009 & 0.007 & 0.008 \\
& $(0.006)$ & $(0.005)$ & $(0.007)$ \\
Year10AfterPP & 0.015 & 0.010 & 0.017 \\
& $(0.007)$ & $(0.005)$ & $(0.008)$ \\
Year15AfterPP & 0.018 & 0.014 & 0.019 \\
& $(0.008)$ & $(0.008)$ & $(0.009)$ \\
\hline Observations & 10,760 & 14,960 & 21,640 \\
Number of firms & 538 & 748 & 1,082 \\
Sample & Balanced & Balanced & Balanced \\
\hline Firm FE & Yes & Yes & Yes \\
Year FE & Yes & Yes & Yes \\
\hline \hline
\end{tabular}

Notes. Coefficients estimated from equation 1 for 538 firms that applied for management transfer (Panel A), 748 firms that applied for technology transfer (Panel B), and 1,082 firms that applied for the combined management and technology transfers (Panel C) and survived in the 15 years after the Productivity Program. Data are provided at the firm level. The dependent variable is log markup estimated as described in Appendix D. Standard errors are block-bootstrapped at the province level with 200 replications. 
Table D.5-: Effects of the Productivity Program after Controlling for Variation in Markups

\begin{tabular}{|c|c|c|c|c|c|c|c|c|c|}
\hline & \multicolumn{3}{|c|}{ A.Management } & \multicolumn{3}{|c|}{ B. Technology } & \multicolumn{3}{|c|}{ C. Combined } \\
\hline & $\begin{array}{l}\text { Log Sales } \\
(1)\end{array}$ & $\begin{array}{l}\text { Log Employment } \\
(2)\end{array}$ & $\begin{array}{l}\text { Log TFPR } \\
(3)\end{array}$ & $\begin{array}{l}\text { Log Sales } \\
(4)\end{array}$ & $\begin{array}{l}\text { Log Employment } \\
(5)\end{array}$ & $\begin{array}{l}\log \text { TFPR } \\
(6)\end{array}$ & $\begin{array}{l}\text { Log Sales } \\
(7)\end{array}$ & $\begin{array}{l}\text { Log Employment } \\
(8)\end{array}$ & $\begin{array}{l}\text { Log TFPR } \\
(9)\end{array}$ \\
\hline Year1AfterPP & $\begin{array}{l}0.058 \\
(0.012)\end{array}$ & $\begin{array}{l}0.009 \\
(0.015)\end{array}$ & $\begin{array}{l}0.131 \\
(0.031)\end{array}$ & $\begin{array}{l}0.006 \\
(0.009)\end{array}$ & $\begin{array}{l}0.012 \\
(0.017)\end{array}$ & $\begin{array}{l}0.015 \\
(0.023)\end{array}$ & $\begin{array}{l}0.086 \\
(0.016)\end{array}$ & $\begin{array}{l}0.038 \\
(0.020)\end{array}$ & $\begin{array}{l}0.180 \\
(0.043)\end{array}$ \\
\hline Year5AfterPP & $\begin{array}{l}0.107 \\
(0.016)\end{array}$ & $\begin{array}{l}0.054 \\
(0.017)\end{array}$ & $\begin{array}{l}0.195 \\
(0.032)\end{array}$ & $\begin{array}{l}0.037 \\
(0.012)\end{array}$ & $\begin{array}{l}0.035 \\
(0.021)\end{array}$ & $\begin{array}{l}0.068 \\
(0.025)\end{array}$ & $\begin{array}{l}0.235 \\
(0.049)\end{array}$ & $\begin{array}{l}0.157 \\
(0.041)\end{array}$ & $\begin{array}{l}0.313 \\
(0.042)\end{array}$ \\
\hline Year10AfterPP & $\begin{array}{l}0.184 \\
(0.044)\end{array}$ & $\begin{array}{l}0.189 \\
(0.040)\end{array}$ & $\begin{array}{l}0.279 \\
(0.051)\end{array}$ & $\begin{array}{l}0.059 \\
(0.017)\end{array}$ & $\begin{array}{l}0.064 \\
(0.028)\end{array}$ & $\begin{array}{l}0.101 \\
(0.046)\end{array}$ & $\begin{array}{l}0.288 \\
(0.065)\end{array}$ & $\begin{array}{l}0.331 \\
(0.058)\end{array}$ & $\begin{array}{l}0.428 \\
(0.061)\end{array}$ \\
\hline Year15AfterPP & $\begin{array}{l}0.304 \\
(0.059)\end{array}$ & $\begin{array}{l}0.281 \\
(0.054)\end{array}$ & $\begin{array}{l}0.378 \\
(0.055)\end{array}$ & $\begin{array}{l}0.058 \\
(0.028)\end{array}$ & $\begin{array}{l}0.068 \\
(0.030)\end{array}$ & $\begin{array}{l}0.097 \\
(0.039)\end{array}$ & $\begin{array}{l}0.417 \\
(0.106)\end{array}$ & $\begin{array}{l}0.457 \\
(0.099)\end{array}$ & $\begin{array}{l}0.555 \\
(0.054)\end{array}$ \\
\hline Observations & 10,760 & 10,760 & 10,760 & 14,960 & 14,960 & 14,960 & 21,640 & 21,640 & 21,640 \\
\hline Number of firms & 538 & 538 & 538 & 748 & 748 & 748 & 1,082 & 1,082 & 1,082 \\
\hline Sample & Balanced & Balanced & Balanced & Balanced & Balanced & Balanced & Balanced & Balanced & Balanced \\
\hline Firm FE & Yes & Yes & Yes & Yes & Yes & Yes & Yes & Yes & Yes \\
\hline Year FE & Yes & Yes & Yes & Yes & Yes & Yes & Yes & Yes & Yes \\
\hline
\end{tabular}

Notes. Coefficients estimated from equation 1 for 538 firms that applied for management transfer (Panel A), 748 firms that applied for technology transfer (Panel B), and 1,082 firms that applied for the combined management and technology transfers (Panel C) and survived in the 15 years after the Productivity Program. Data are provided at the firm level. The dependent variables are logged deflated Sales converted from 1951 Italian lira to 2010 euro and exchanged at 0.780 euro = USD 1 (columns 1, 4, and 7); logged Employment, reporting the number of employees per firm (columns 2, 5, and 8); and logged TFPR, estimated using the Ackerberg, Caves and Frazer (2006) method (columns 3, 6, and 9). Standard errors are block-bootstrapped at the province level with 200 replications. denotes $1 \%$, denotes $5 \%$, and denotes $10 \%$ significance. 
Table D.6 - Verifying Balance in Terms of Firms' Characteristics and Outcomes with IPTW

\begin{tabular}{|c|c|c|c|c|c|c|c|c|c|c|}
\hline & \multicolumn{3}{|c|}{ Management (1-3) } & \multicolumn{3}{|c|}{ Technology (4-6) } & \multicolumn{3}{|c|}{ Combined (7-9) } & \multirow{3}{*}{$\begin{array}{l}\text { All Transfers } \\
F \text {-statistics } \\
\text { Equality } \\
\text { All Means } \\
\end{array}$} \\
\hline & \multicolumn{2}{|c|}{$\begin{array}{c}\text { Treated } \\
\text { Provinces }\end{array}$} & \multirow[t]{2}{*}{ Difference } & \multicolumn{2}{|c|}{$\begin{array}{c}\text { Treated } \\
\text { Provinces }\end{array}$} & Difference & \multicolumn{2}{|c|}{$\begin{array}{c}\text { Treated } \\
\text { Provinces }\end{array}$} & Difference & \\
\hline & Yes & No & & Yes & No & & Yes & No & & \\
\hline & (1) & $(2)$ & (3) & (4) & (5) & (6) & (7) & (8) & (9) & $(10)$ \\
\hline Employees per firm & $\begin{array}{l}40.92 \\
(33.45)\end{array}$ & $\begin{array}{l}39.89 \\
(34.98)\end{array}$ & $\begin{array}{l}1.03 \\
(4.37)\end{array}$ & $\begin{array}{l}40.02 \\
(35.87)\end{array}$ & $\begin{array}{l}39.22 \\
(37.41)\end{array}$ & $\begin{array}{l}0.80 \\
(1.44)\end{array}$ & $\begin{array}{l}39.43 \\
(39.61)\end{array}$ & $\begin{array}{l}40.67 \\
(37.23)\end{array}$ & $\begin{array}{l}-1.24 \\
(3.75)\end{array}$ & 0.567 \\
\hline Current assets (k USD) & $\begin{array}{l}1,833.45 \\
(2,738.26)\end{array}$ & $\begin{array}{l}1,836.41 \\
(2,889.44)\end{array}$ & $\begin{array}{l}-2.96 \\
(3.46)\end{array}$ & $\begin{array}{l}1,831.87 \\
(2,809.31)\end{array}$ & $\begin{array}{l}1,834.89 \\
(2,983.29)\end{array}$ & $\begin{array}{l}-3.02 \\
(4.39)\end{array}$ & $\begin{array}{l}1,835.89 \\
(2,578.10)\end{array}$ & $\begin{array}{l}1,832.38 \\
(2,493.1)\end{array}$ & $\begin{array}{l}3.51 \\
(15.93)\end{array}$ & 0.732 \\
\hline Annual sales (k USD) & $\begin{array}{l}915.69 \\
(1,342.28)\end{array}$ & $\begin{array}{l}913.91 \\
(1,904.39)\end{array}$ & $\begin{array}{l}1.78 \\
(1.90)\end{array}$ & $\begin{array}{l}911.90 \\
(1,509.29)\end{array}$ & $\begin{array}{l}914.39 \\
(1,432.91)\end{array}$ & $\begin{array}{l}-2.49 \\
(3.48)\end{array}$ & $\begin{array}{l}915.22 \\
(1,783.91)\end{array}$ & $\begin{array}{l}918.65 \\
(1,678.01)\end{array}$ & $\begin{array}{l}-3.43 \\
(4.39)\end{array}$ & 0.804 \\
\hline Productivity (log TFPR) & $\begin{array}{l}2.65 \\
(0.49)\end{array}$ & $\begin{array}{l}2.58 \\
(0.46)\end{array}$ & $\begin{array}{l}0.07 \\
(0.09)\end{array}$ & $\begin{array}{l}2.51 \\
(0.42)\end{array}$ & $\begin{array}{l}2.56 \\
(0.41)\end{array}$ & $\begin{array}{l}-0.05 \\
(0.08)\end{array}$ & $\begin{array}{l}2.60 \\
(0.49)\end{array}$ & $\begin{array}{l}2.66 \\
(0.50)\end{array}$ & $\begin{array}{l}-0.06 \\
(0.07)\end{array}$ & 0.421 \\
\hline Export & $\begin{array}{l}0.14 \\
(0.37)\end{array}$ & $\begin{array}{l}0.13 \\
(0.39)\end{array}$ & $\begin{array}{l}0.02 \\
(0.08)\end{array}$ & $\begin{array}{l}0.12 \\
(0.33)\end{array}$ & $\begin{array}{l}0.14 \\
(0.38)\end{array}$ & $\begin{array}{l}-0.03 \\
(0.05)\end{array}$ & $\begin{array}{l}0.14 \\
(0.31)\end{array}$ & $\begin{array}{l}0.13 \\
(0.39)\end{array}$ & $\begin{array}{l}0.01 \\
(0.05)\end{array}$ & 0.480 \\
\hline Family-managed & $\begin{array}{l}0.25 \\
(0.46)\end{array}$ & $\begin{array}{l}0.24 \\
(0.48)\end{array}$ & $\begin{array}{l}0.01 \\
(0.07)\end{array}$ & $\begin{array}{l}0.24 \\
(0.49)\end{array}$ & $\begin{array}{l}0.23 \\
(0.41)\end{array}$ & $\begin{array}{l}0.01 \\
(0.08)\end{array}$ & $\begin{array}{l}0.25 \\
(0.39)\end{array}$ & $\begin{array}{l}0.25 \\
(0.49)\end{array}$ & $\begin{array}{l}-0.02 \\
(0.09)\end{array}$ & 0.298 \\
\hline
\end{tabular}

Notes. Columns $1,2,4,5,7$, and 8 report the means of firm covariates weighting each observation by the inverse of its propensity score. Columns 3,6 , and 9 report the $\beta_{j}$ coefficients estimated from outcome ${ }_{i}=\sum_{j=1}^{3} \alpha_{j} \operatorname{Transfer}_{i}^{j}+\sum_{j=1}^{3} \beta_{j}\left(\operatorname{Transfer}_{i}^{j}\right.$. Treated Province Tr $_{p}+\lambda_{r}+\epsilon_{i}$ in 1951 , where Transfer $^{j}$ is an indicator for firms that applied for management transfer for $j=1$, for technology transfer for $j=2$, and for the combined management and technology transfers for $j=3$, Treated Province is an indicator for firms located in a treated province, and $\lambda_{r}$ is pilot region fixed effects, in which each observation is weighted by the inverse of its propensity score. Column 10 reports the $F$-statistics of testing the null hypothesis of equality between the six coefficients. Standard errors are block-bootstrapped using 200 replications. Firm covariates are: Employees per firm reports the number of employees per firm: Current assets and Annual sales are in 2010 USD, reevaluated from 1951 to 2010 values at 1 lira $=30.884$ euros and exchanged at 0.780 euro $=$ USD 1; Productivity $(\log$ TFPR) is the logarithm of total factor productivity revenue, estimated using the Ackerberg, Caves and Frazer (2006) method; Export and Family-managed are indicators that equal one if, respectively, a firm exported and was family-managed. 


\section{E. Estimation of the Production Function}

I assume a Cobb-Douglas production function

$$
Y_{i t}=A_{i t} K_{i t}^{\beta_{k}} L_{i t}^{\beta_{l}}
$$

where $Y_{i t}$ is the value added of firm $i$ in period $t, K_{i t}$ and $L_{i t}$ are inputs of capital and labor, and $A_{i t}$ is the Hicksian-neutral efficiency level. Taking natural logs, equation E.1 results in the linear production function

$$
y_{i t}=\beta_{0}+\beta_{k} k_{i t}+\beta_{l} l_{i t}+\underbrace{\omega_{i t}+\eta_{i t}}_{\epsilon_{i t}}
$$

where lower-case letters refer to natural logarithms, $\beta_{0}$ measures the mean efficiency level across firms and over time, $\epsilon_{i t}$ is the time- and producer-specific deviation from that mean, which can then be further decomposed into an observable (or at least predictable) $\omega_{i t}$ and unobservable component $\eta_{i t}$. $\omega_{i t}$ is a productivity shock (which may include, for instance, machinery breakdown, demand shock, and managerial skills) and $\eta_{t}$ is an i.i.d. component, representing unexpected deviations from the mean due to measurement error, unexpected delays, or other external circumstances.

The major econometric issue of estimating equation E.2 is that the firm's optimal choice of inputs $k_{i t}$ and $l_{i t}$ is generally correlated with the observed productivity shock $\omega_{i t}$, which renders OLS estimates of the $\beta$ 's biased and inconsistent.

Possible solutions for this problem include using instrumental variable estimation techniques or controlling for firm fixed effects. In practice, however, these solutions have not worked well. Natural instruments, such as input prices if firms are operating in competitive input markets, are often not observed or do not vary enough across firms, and fixed effects estimation requires the strong assumption that the unobservables are constant across time, i.e., $\omega_{i t}=\omega_{i t-1} \forall t$ (Ackerberg, Caves and Frazer, 2006). The dynamic panel literature extends the fixed effects literature to allow for more sophisticated error structures (Bond and Soderbom, 2005). For instance, it is possible to assume that $\omega$ follows an $\operatorname{AR}(1)$ process, i.e., $\omega_{i t}=\rho \omega_{i t-1}+\xi_{i t}$. Since the innovation in $\omega_{i t}, \xi_{i t}$, occurs after time $t-1$, it may not be correlated with inputs dated $t-1$ and earlier (Ackerberg, Caves and Frazer, 2006), and this is used to derive the moment conditions. ${ }^{6}$

Other solutions, such as those advocated by Olley and Pakes (1996) and Levinsohn and Petrin (2003), involve a more structural approach and use investment or intermediate inputs proxy for productivity shocks. Specifically, they assume

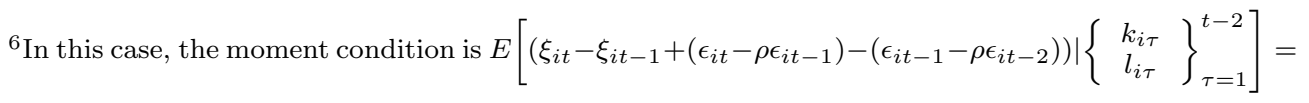
0. 
that labor is the nondynamic input, capital is the dynamic input, and that

$$
m_{i t}=f_{t}\left(k_{i t}, \omega_{i t}\right)
$$

where $m_{i t}$ is investment in the Olley and Pakes (1996)'s method and intermediate inputs in the Levinsohn and Petrin (2003)'s method and is function of capital $k_{i t}$ and productivity $\omega_{i t}{ }^{7}$

Assuming that E.3 is invertible, then

$$
\omega_{i t}=f_{t}^{-1}\left(k_{i t}, m_{i t}\right)
$$

and substituting in equation E.2,

$$
y_{i t}=\beta_{0}+\beta_{k} k_{i t}+\beta_{l} l_{i t}+f_{t}^{-1}\left(k_{i t}, m_{i t}\right)+\eta_{i t}
$$

where $f_{t}^{-1}$ is treated as nonparametric. The estimation consists of two steps. First, equation E.5 is estimated by using semiparametric techniques. This allows estimating $\beta_{l}$, but does not identify $\beta_{k}$, since it is collinear with the nonparametric function. Second, assuming that $\omega$ follows a first-order Markov process implies that

$$
\omega_{i t}=E\left[\omega_{i t} \mid m_{i t-1}\right]+\xi_{i t}=E\left[\omega_{i t} \mid \omega_{i t-1}\right]+\xi_{i t}
$$

where $\xi$ is the "innovation" component of $\omega$, such that $E\left[\xi_{i t} \mid m_{i t-1}\right]=0$. Since capital at time $t$ is decided at time $t-1, E\left[\xi_{i t} \mid k_{i t}\right]=0 .{ }^{8}$ Variation in $k_{i t}$ conditional on $\omega_{i t-1}$ is the exogenous variation used to identify $\beta_{k}$, which is estimated via GMM using the following moment conditions:

$$
\frac{1}{T} \frac{1}{N} \sum_{t} \sum_{i} \xi_{i t}\left(\beta_{k}\right) \cdot k_{i t}
$$

In this paper, I use the method proposed by Ackerberg, Caves and Frazer (2006), which is based on the Olley and Pakes (1996) and Levinsohn and Petrin (2003) methods, but solves the possible collinearity problem between labor and investment or intermediate inputs. This collinearity problem may arise because labor and investment or intermediate inputs have the same data generation process (DGP). Therefore, it is not possible to simultaneously estimate a fully nonparametric (time-varying) function of $\left(\omega_{i t}, k_{i t}\right)$ along with a coefficient on a variable that is only a (time-varying) function of those same variables $\left(\omega_{i t}, k_{i t}\right)$. The Ackerberg, Caves and Frazer (2006) method assumes that $l_{i t}$ is chosen by firms at time

\footnotetext{
${ }^{7}$ Petrin, Poi and Levinsohn (2004) propose to use intermediate inputs rather than investment as a proxy for productivity shocks, because investment is lumpy due to substantial adjustment costs and, so, it might not smoothly respond to the productivity shock.

${ }^{8}$ Olley and Pakes (1996) also control for selection, by introducing an exit rule for firms.
} 
$t-b(0<b<1)$, after $k_{i t}$ was chosen at time $t-1$, but before $m_{i t}$ being chosen at time $t$. In this setup,

$$
m_{i t}=f_{t}\left(\omega_{i t}, k_{i t}, l_{i t}\right)
$$

In the first stage, $\beta_{l}$ is not identified, but it is possible to estimate $\Phi_{t}\left(m_{i t}, k_{i t}, l_{i t}\right)=$ $\beta_{k} k_{i t}+\beta_{l} l_{i t}+f_{t}^{-1}\left(m_{i t}, k_{i t}, l_{i t}\right)$, which represents output net of the untransmitted shock $\eta_{i t}$. In the second stage, the moment condition on capital is $E\left[\xi_{i t} \mid k_{i t}\right]=0$ (which comes from $\omega$ following a first order Markov process and implies $E\left[\xi_{i t}\right.$. $\left.k_{i t}\right]=0$ ) and the moment condition on labor is $E\left[\begin{array}{cc}\xi_{i t} & k_{i t} \\ & l_{i t-1}\end{array}\right]=0$ (since $l_{i t-1}$ was

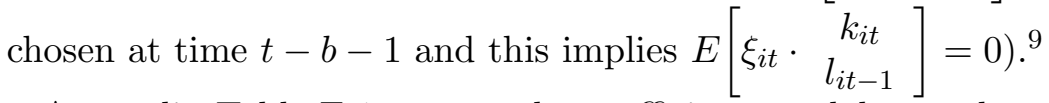

Appendix Table E.1 reports the coefficients on labor and capital estimated by using the Ackerberg, Caves and Frazer (2006) method, separately for each manufacturing industry. To check the extent to which the Ackerberg, Caves and Frazer (2006) estimates differ from other estimates, I also report the labor and capital coefficients estimated with the OLS, the factor shares (Solow's residuals), the Levinsohn and Petrin (2003) method, and the dynamic panel method. The OLS and factor shares calculations tend to underestimate the coefficients on capital compared to the Ackerberg, Caves and Frazer (2006)'s coefficients, while the Levinsohn and Petrin (2003) method tends to overestimate it. However, the coefficients are roughly comparable across the different estimation methods and in each industry I cannot reject the null hypothesis of constant return to scale. ${ }^{10}$

\section{A. Definition of the Variables}

To estimate the production function in equation E.2, I use the following variables:

- value added: measured as the difference between firm deflated total income and intermediate inputs. The deflator used is the year-industry deflator, with base-year 1946.

- labor: measured by number of employees.

- capital: measured by firm capital stock. To obtain a measure of firm capital stock from the fixed gross assets $(f g a)$ reported in the balance sheets, I use

\footnotetext{
${ }^{9}$ Compared with the dynamic panel approach, the Ackerberg, Caves and Frazer (2006) method allows estimating $\omega$ separately from $\epsilon$. This has two major implications: (1) in the Ackerberg, Caves and Frazer (2006)'s method $\omega$ can follow a first-order Markov process not necessarily linear; (2) the variance of a GMM estimator is proportional to the variance of the moment condition being used, so Ackerberg, Caves and Frazer (2006) method is more efficient. However, the GMM estimator can allow for a fixed effect $\alpha_{i}$ in addition to $\omega_{i t}$, allows for $\epsilon_{i t}$ to be correlated over time and allows for $\omega$ following a higher than first order Markov process, as long as this process is linear (Ackerberg, Caves and Frazer (2006)).

${ }^{10} \mathrm{I}$ measure firm output by using deflated value added, which might not reflect the ranking of firms in their productivity if they charge different markups.
} 
the Perpetual Inventory Method (PIM). First, I compute investment $I$ as the difference between the deflated current and the lagged fga. This enables me to use the PIM formula

$$
P_{t+1} K_{t+1}=P_{t+1}(1-\delta) P_{t} K_{t}+P_{t+1} I_{t+1}
$$

where $K$ is the quantity of capital, $P$ is its price (set equal to the interest rate on credit for 1946 to 1950 and to the national industry credit rate for 1951 to 1970 ), $I$ is investment, and $\delta$ is the depreciation rate (set equal to 6.5 percent, according to the average estimated life of machine of 15 years (ISTAT, 2012). However, this procedure is valid only if the base-year capital stock (the first year in the data for a given firm) can be written as $P_{0} K_{0}$ , which is not the case here because in the balance sheets $f g a$ is reported at its historic cost. To estimate its value at replacement cost, I use the $R^{G}$ factor suggested by Balakrishnan, Pushpangadan and Suresh Babu (2000):

$$
R^{G}=\frac{\left[(1+g)^{\tau+1}-1\right](1+\pi)^{\tau}[(1+g)(1+\pi)-1]}{g\left\{[(1+g)(1+\pi)]^{\tau+1}-1\right\}}
$$

where $\tau$ is the average life of machines (assumed to be 15 years, according to ISTAT, 2012), $\pi$ is the average capital price $\frac{P_{t}}{P_{t-1}}$ from 1946 to 1973 (equal to 1.00255), and $g$ is the (assumed constant) real investment growth rate $\frac{I_{t}}{I_{t-1}}$ from 1946 to 1973 (equal to 1.062272). I multiply fga in the base year 1946 by $R^{G}$ to convert capital to replacement costs at current prices, which I then deflate using the price index for machinery and machine tools to express it in real terms. Finally, I apply formula E.8. 
Table E.1-: Estimation of Production Function

\begin{tabular}{|c|c|c|c|c|c|c|c|c|c|}
\hline & \multicolumn{3}{|c|}{ I. Food } & \multicolumn{3}{|c|}{ II. Textile } & \multicolumn{3}{|c|}{ III.Wood } \\
\hline & $\begin{array}{l}\beta_{l} \\
(1)\end{array}$ & $\begin{array}{l}\beta_{k} \\
(2) \\
\end{array}$ & $\begin{array}{l}p \text {-value } \\
\beta_{l}+\beta_{k}=1 \\
(3)\end{array}$ & $\begin{array}{l}\beta_{l} \\
(4)\end{array}$ & $\begin{array}{l}\beta_{k} \\
(5) \\
\end{array}$ & $\begin{array}{l}p \text {-value } \\
\beta_{l}+\beta_{k}=1 \\
(6)\end{array}$ & $\begin{array}{l}\beta_{l} \\
(7) \\
\end{array}$ & $\begin{array}{l}\beta_{k} \\
(8) \\
\end{array}$ & $\begin{array}{l}p \text {-value } \\
\beta_{l}+\beta_{k}=1 \\
(9)\end{array}$ \\
\hline $\mathrm{ACF}$ & $\begin{array}{l}0.58 \\
(0.12)\end{array}$ & $\begin{array}{l}0.44 \\
(0.11)\end{array}$ & 0.367 & $\begin{array}{l}0.67 \\
(0.15)\end{array}$ & $\begin{array}{l}0.35 \\
(0.07)\end{array}$ & 0.451 & $\begin{array}{l}0.55 \\
(0.18)\end{array}$ & $\begin{array}{l}0.47 \\
(0.15)\end{array}$ & 0.246 \\
\hline OLS & $\begin{array}{l}0.61 \\
(0.13)\end{array}$ & $\begin{array}{l}0.40 \\
(0.14)\end{array}$ & 0.281 & $\begin{array}{l}0.70 \\
(0.13)\end{array}$ & $\begin{array}{l}0.33 \\
(0.10)\end{array}$ & 0.342 & $\begin{array}{l}0.56 \\
(0.12)\end{array}$ & $\begin{array}{l}0.42 \\
(0.11)\end{array}$ & 0.358 \\
\hline Factor Shares & 0.55 & 0.45 & & 0.64 & 0.36 & & 0.57 & 0.43 & \\
\hline LP & $\begin{array}{l}0.56 \\
(0.11)\end{array}$ & $\begin{array}{l}0.47 \\
(0.09)\end{array}$ & 0.452 & $\begin{array}{l}0.63 \\
(0.12)\end{array}$ & $\begin{array}{l}0.39 \\
(0.08)\end{array}$ & 0.246 & $\begin{array}{l}0.50 \\
(0.11)\end{array}$ & $\begin{array}{l}0.51 \\
(0.13)\end{array}$ & 0.435 \\
\hline \multirow[t]{3}{*}{ DP } & $\begin{array}{l}0.59 \\
(0.13)\end{array}$ & $\begin{array}{l}0.44 \\
(0.10)\end{array}$ & 0.498 & $\begin{array}{l}0.65 \\
(0.11)\end{array}$ & $\begin{array}{l}0.36 \\
(0.09)\end{array}$ & 0.377 & $\begin{array}{l}0.57 \\
(0.08)\end{array}$ & $\begin{array}{l}0.46 \\
(0.11)\end{array}$ & 0.239 \\
\hline & \multicolumn{3}{|c|}{ IV. Machinery } & \multicolumn{3}{|c|}{ V. Minerals } & \multicolumn{3}{|c|}{ VI. Chemicals } \\
\hline & $\begin{array}{l}\beta_{l} \\
(10)\end{array}$ & $\begin{array}{l}\beta_{k} \\
(11)\end{array}$ & $\begin{array}{l}p \text {-value } \\
\beta_{l}+\beta_{k}=1 \\
(12)\end{array}$ & $\begin{array}{l}\beta_{l} \\
(13)\end{array}$ & $\begin{array}{l}\beta_{k} \\
(14)\end{array}$ & $\begin{array}{l}p \text {-value } \\
\beta_{l}+\beta_{k}=1 \\
(15)\end{array}$ & $\begin{array}{l}\beta_{l} \\
(16)\end{array}$ & $\begin{array}{l}\beta_{k} \\
(17)\end{array}$ & $\begin{array}{l}p \text {-value } \\
\beta_{l}+\beta_{k}=1 \\
(18)\end{array}$ \\
\hline $\mathrm{ACF}$ & $\begin{array}{l}0.62 \\
(0.13)\end{array}$ & $\begin{array}{l}0.39 \\
(0.09)\end{array}$ & 0.539 & $\begin{array}{l}0.61 \\
(0.08)\end{array}$ & $\begin{array}{l}0.42 \\
(0.15)\end{array}$ & 0.371 & $\begin{array}{l}0.65 \\
(0.21)\end{array}$ & $\begin{array}{l}0.34 \\
(0.11)\end{array}$ & 0.654 \\
\hline OLS & $\begin{array}{l}0.64 \\
(0.09)\end{array}$ & $\begin{array}{l}0.35 \\
(0.11)\end{array}$ & 0.432 & $\begin{array}{l}0.62 \\
(0.10)\end{array}$ & $\begin{array}{l}0.40 \\
(0.14)\end{array}$ & 0.254 & $\begin{array}{l}0.66 \\
(0.19)\end{array}$ & $\begin{array}{l}0.32 \\
(0.11)\end{array}$ & 0.348 \\
\hline Factor Shares & 0.65 & 0.35 & & 0.64 & 0.36 & & 0.62 & 0.38 & \\
\hline LP & $\begin{array}{l}0.57 \\
(0.11)\end{array}$ & $\begin{array}{l}0.42 \\
(0.13)\end{array}$ & 0.394 & $\begin{array}{l}0.63 \\
(0.14)\end{array}$ & $\begin{array}{l}0.44 \\
(0.17)\end{array}$ & 0.365 & $\begin{array}{l}0.63 \\
(0.13)\end{array}$ & $\begin{array}{l}0.38 \\
(0.13)\end{array}$ & 0.493 \\
\hline DP & $\begin{array}{l}0.61 \\
(0.12)\end{array}$ & $\begin{array}{l}0.40 \\
(0.15)\end{array}$ & 0.453 & $\begin{array}{l}0.62 \\
(0.15)\end{array}$ & $\begin{array}{l}0.42 \\
(0.15)\end{array}$ & 0.410 & $\begin{array}{l}0.67 \\
(0.21)\end{array}$ & $\begin{array}{l}0.34 \\
(0.12)\end{array}$ & 0.352 \\
\hline
\end{tabular}

Notes. Coefficients on labor $\left(\beta_{l}\right)$ and capital $\left(\beta_{k}\right)$ estimated with the Ackerberg, Caves and Frazer (2006) method (ACF), OLS, factor shares (Solow's residuals), Petrin, Poi and Levinsohn (2004) (LP), and dynamic-panel method (DP), separately for each manufacturing industry. Columns 3, 6, 9, 12, 15, and 18 report the $p$-value of testing constant return to scale (CRS) $\beta_{l}+\beta_{k}=1$. The sample include 6,065 Italian firms eligible to apply for the Productivity Program. Data are provided at the firm level. denotes $1 \%$, denotes $5 \%$, and denotes $10 \%$ significance. 


\section{REFERENCES}

Ackerberg, Daniel A., Kevin Caves, and Garth Frazer. 2006. "Structural Identification of Production Functions."

Balakrishnan, Pulapre K., K. Pushpangadan, and M. Suresh Babu. 2000. "Trade Liberalisation and Productivity Growth in Manufacturing: Evidence from Firm-Level Panel Data." Economic and Political Weekly, 35(41): 3679-3682.

Bond, Stephen, and Mans Soderbom. 2005. "Adjustment Costs and the Identification of Cobb Douglas Production Functions."

De Loecker, Jan, and Frederic Warzynski. 2012. "Markups and firm-level export status." American Economic Review, 102(6): 2437-2471.

ISTAT. 2012. "Nota Metodologica sulle Misure di Produttività."

Klette, Tor Jakob, and Zvi Griliches. 1996. "The Inconsistency of Common Scale Estimators when Output Prices Are Unobserved and Endogenous." Journal of Applied Econometrics, 11(4): 343-61.

Lee, David S. 2009. "Training, wages, and sample selection: Estimating sharp bounds on treatment effects." Review of Economic Studies, 76: 1071-1102.

Levinsohn, James, and Amil Petrin. 2003. "Production Functions Estimating to Control for Using Inputs Unobservables." Review of Economic Studies, 70(2): 317-341.

Olley, G. Steven, and Ariel Pakes. 1996. "The Dynamics of Productivity in the Telecommunications Equipment Industry." Econometrica, 64(6): 12631297.

Petrin, Amil, Brian P. Poi, and James Levinsohn. 2004. "Production Function Estimation in Stata Using Input to Estimate the Unobservables." Stata Journal, 4(2): 113-123.

Zamagni, Vera. 1997. Come Perdere la Guerra e Vincere la Pace. L'Economia Italiana tra Guerra e Dopoguerra: 1938-1947. Bologna:Il Mulino. 